\title{
Mitochondrial-Derived Compartments Facilitate Cellular Adaptation to Amino Acid Stress
}

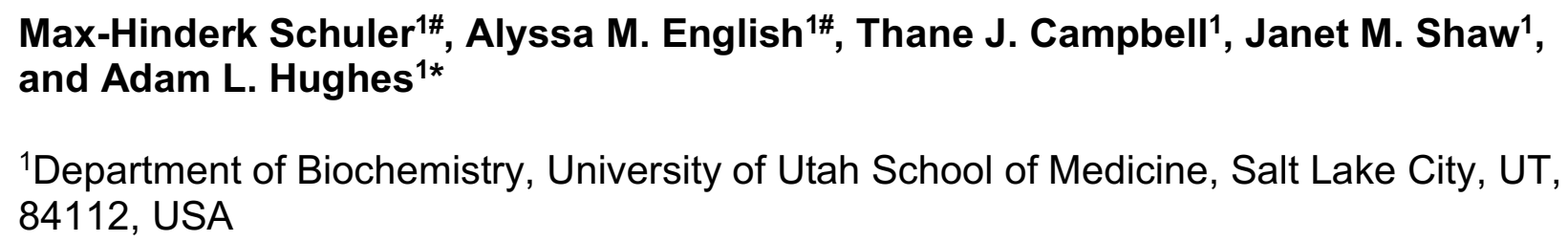

\#These authors contributed equally to this work

${ }^{*}$ Correspondence:

Department of Biochemistry

University of Utah School of Medicine

15 N. Medical Drive East

20 RM 4100

21 Salt Lake City, UT, 84112

22 Phone: 801-581-2481

23 Fax: 801-581-7959

24 Email: hughes@biochem.utah.edu 


\section{SUMMARY}

Amino acids are essential building blocks of life. However, increasing evidence

50 suggests that elevated amino acids cause cellular toxicity associated with numerous

51 metabolic disorders. How cells cope with elevated amino acids remains poorly

52 understood. Here, we show that a previously identified cellular structure, the

53 mitochondrial-derived compartment (MDC), is a dynamic, lumen-containing organelle

54 that functions to protect cells from amino acid stress. In response to amino acid

55 elevation, MDCs are generated from mitochondria, where they selectively sequester

56 and remove Tom70, a surface receptor required for import of nutrient carriers of the

57 SLC25 family. MDC formation is regulated by levels of mitochondrial carriers, and its

58 activation by amino acids occurs simultaneously with removal of plasma membrane-

59 localized transporters via the multi-vesicular body (MVB) pathway. Combined loss of

60 MDC and MVB formation renders cells sensitive to elevated amino acids, suggesting

61 these pathways operate as a coordinated network to protect cells from amino acid

62 toxicity.

63

\section{KEYWORDS}

65 Mitochondria, vacuole, amino acid, MDC, branched-chain amino acids, nutrient

66 transporter, lysosome

67

68

69 


\section{INTRODUCTION}

72 Amino acids are essential metabolites utilized as fuel sources, signaling molecules, and

73 precursors for the biosynthesis of proteins, lipids, heme, nucleotides, and other cellular

74 molecules (Ljungdahl and Daignan-Fornier, 2012). As with most metabolites, cells must

75 maintain amino acid homeostasis at all times, and they are equipped with numerous

76 systems that monitor amino acid concentrations and adjust the rates of amino acid

77 acquisition, storage, and utilization accordingly (Efeyan et al., 2015). To date, we know

78 a great deal about the impact of amino acid starvation on cells, as well as the signaling pathways and remodeling systems such as autophagy that operate to maintain cellular health when amino acids are in short supply (Efeyan et al., 2015; Rabinowitz and White,

81 2010). On the other hand, elevated amino acids can lead to cellular toxicity and are

82 associated with aging and numerous metabolic disorders, including insulin resistance

83 and a host of inborn errors of amino acid metabolism (Aliu et al., 2018; Newgard et al.,

84 2009; Ruiz et al., 2017; Soultoukis and Partridge, 2016). In contrast to our knowledge of

85 cellular adaptation to amino acid starvation, we understand little about the mechanisms

86 that drive amino acid toxicity and pathways that protect cells from amino acid

87 overabundance stress (Wellen and Thompson, 2010).

We recently discovered that the yeast lysosome (vacuole) functions as a

89 safeguard against cellular amino acid toxicity through its ability to import and sequester

90 amino acids (Hughes and Gottschling, 2012; Hughes et al., 2020). Defects in vacuolar

91 amino acid compartmentation impair mitochondrial respiration and negatively impact

92 cellular health (Hughes and Gottschling, 2012; Hughes et al., 2020). In addition, prior

93 work in yeast has shown that the regulation of plasma membrane (PM)-localized 
94 nutrient transporters via the multi-vesicular body (MVB) pathway also serves as a key

95 mechanism to control cellular nutrient uptake, and protects cells from amino acid toxicity

96 (Katzmann et al., 2002; Risinger et al., 2006; Rubio-Texeira and Kaiser, 2006; Ruiz et

97 al., 2017). Beyond lysosomes and MVBs, additional mechanisms cells utilize to protect

98 themselves from amino acid toxicity remain unclear.

While investigating the impact of lysosome failure on mitochondrial health, we identified a new cellular structure that forms from mitochondria when lysosomal

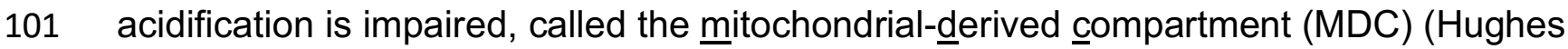
et al., 2016). Upon formation, MDCs selectively incorporate a number of mitochondrial proteins including Tom70, an outer membrane (OM) import receptor for mitochondrial

104 nutrient transporters (Sollner et al., 1990). By contrast, MDCs exclude most other 105 mitochondrial proteins, including those in the mitochondrial matrix, the intermembrane 106 space (IMS), and the majority of inner membrane (IM) proteins. After formation, MDCs 107 are released from mitochondria via mitochondrial fission and are degraded by 108 autophagy (Hughes et al., 2016). Currently, we know that MDCs are Tom70-enriched 109 foci that associate with mitochondria when cells lose lysosome acidification. Beyond 110 that, we understand little about the dynamics and regulation of MDC formation, as well 111 as the function of this new cellular compartment.

113 that are generated in response to perturbations in intracellular amino acid homeostasis.

114 Specifically, we find that high levels of branched-chain amino acids (BCAAs) and their

115 breakdown products promote MDC formation. Our data indicate that MDCs sequester

116 Tom70 away from mitochondria and might thereby provide cells with a mechanism to 
117 fine-tune levels of mitochondrial nutrient transporters in response to changes in cellular

118 amino acid load. Consistent with this idea, increasing nutrient transporter levels on

119 mitochondria via Tom70 or nutrient transporter overexpression triggers constitutive

120 MDC formation. Finally, we show that MDC formation and removal of nutrient

121 transporters from the PM via the MVB pathway are activated in parallel, and that

122 combined loss of both systems impairs the cell's ability to cope with toxic levels of

123 BCAAs. Overall, our data suggest that MDCs are part of a coordinated cellular program

124 that operates to protect cells from amino acid stress.

125

126 RESULTS

127 MDCs are dynamic, lumen-containing organelles that stably associate with 128 mitochondria

129 We previously identified MDCs in budding yeast, S. cerevisiae, as bright foci that form in 130 association with mitochondria during aging or in response to acute pharmacological

131 disruption of lysosome acidification (Hughes et al., 2016). We confirmed our prior

132 observations by showing that treatment of cells with concanamycin $A$ (concA), a specific

133 inhibitor of the evolutionarily conserved Vacuolar $\mathrm{H}^{+}$-ATPase (V-ATPase) proton pump,

134 triggered formation of foci containing C-terminally GFP tagged Tom70 (Figure 1A). Line-

135 scan analysis demonstrated that MDCs exhibited an enrichment of Tom70 compared to

136 the rest of the mitochondrial tubule and excluded the IM protein Tim50-mCherry

137 (Yamamoto et al., 2002), a subunit of the TIM23 complex that we previously showed

138 was excluded from MDCs (Figure 1A). Disruption of lysosome acidification via auxin-

139 dependent depletion of the V-ATPase subunit Vma2 also induced MDC formation, 
140 indicating that MDCs form in direct response to loss of lysosome acidification (Figures

141 S1A-D). Utilizing super-resolution microscopy, we found that MDCs are organelle-like

142 structures with distinct lumens that reach diameters approaching $\sim 1 \mu \mathrm{m}$ (Figures 1B-C).

143 Serial optical-plane sectioning indicated that MDCs exhibited reduced diameters near

144 the top and bottom and appeared closed at both ends, suggesting they are membrane-

145 bound compartments (Figure 1D). In support of this latter conclusion, we previously

146 showed that MDCs are released from mitochondria by the fission GTPase Dnm1, which

147 acts on membrane-bound organelles (Hughes et al., 2016). MDCs excluded the

148 mitochondrial DNA stain 4',6-diamidino-2-phenylindole (DAPI), and did not incorporate

149 the mitochondrial inner membrane potential-dependent dye tetramethylrhodamine

150 (TMRM), indicating that MDCs do not contain mitochondrial DNA or a membrane

151 potential and are thus distinct structures compared to mitochondria (Figures 1E-F).

152 To elucidate the kinetics and dynamics of MDC formation, we utilized super-

153 resolution time-lapse imaging to visualize MDC formation over a two-hour time period.

154 As shown in Figure 1G and Video S1, MDCs first appeared as small foci with an

155 enrichment of Tom70-GFP compared to the rest of the tubule, usually within 20-30

156 minutes of concA addition (Figure 1G, 26 and 29 minute panels). MDCs remained

157 stably associated with the mitochondrial tubule and grew in size over the 2-hour time-

158 course, with large lumens becoming visible at late stages of formation (Figure $1 \mathrm{G}$ and

159 Video S1). During and after formation, MDCs exhibited dynamic properties including

160 frequent elongation and tubulation (Figures 1G, S1E and Video S2). Tubulation often

161 preceded the appearance of a clear lumen, suggesting this may be a general feature of 
162 MDC formation (Figures $1 \mathrm{G}$ and S1E). Altogether, these data indicate that MDCs are

163 membrane-bound, dynamic organelles capable of stable mitochondrial association.

165 MDC formation is triggered by elevated cellular amino acid load

166 To elucidate the function of MDCs, we sought to identify the signal originating from

167 dysfunctional lysosomes that activates MDC formation. We and others previously

168 showed that mitochondria and lysosomes are functionally linked, and that loss of

169 lysosome acidification impairs mitochondrial respiration (Chen et al., 2020; Dimmer et

al., 2002; Hughes and Gottschling, 2012; Hughes et al., 2020; Merz and Westermann,

171 2009; Ohya et al., 1991; Weber et al., 2020; Yambire et al., 2019). In a recent study, we

172 demonstrated that lysosomes support mitochondrial respiration by spatially

173 compartmentalizing amino acids (Hughes et al., 2020). Loss of V-ATPase function

174 prevents proper storage of amino acids in lysosomes, and causes acute amino acid

175 stress (Figure 2A). This amino acid stress is associated with global cellular rewiring at

176 the mRNA level, with cells turning-down amino acid biosynthesis and upregulating

177 pathways that break down amino acids (Hughes et al., 2020). Furthermore, elevated

178 amino acids cause mitochondrial depolarization by altering the bioavailability of

179 intracellular iron, through an oxidant-based mechanism (Hughes et al., 2020).

$180 \quad$ Based on these prior observations, we reasoned that mitochondrial

181 depolarization, iron deprivation, oxidative stress, or alterations in amino acid pools in V182 ATPase-impaired cells may activate MDC formation. Treatment with the ATP synthase 183 inhibitor oligomycin, mitochondrial depolarizing agents antimycin A and FCCP, hypoxia 184 mimetic $\mathrm{CoCl}_{2}$, the ER stress inducer tunicamycin, or the oxidant hydrogen peroxide did 
not induce MDC formation (Figure S2A). Likewise, MDCs were not induced when cells were treated with the iron chelator BPS (Figure S2B), and iron addition to concA-treated cells, which is sufficient to restore mitochondrial respiration in the absence of a

188 functioning vacuole (Chen et al., 2020; Hughes et al., 2020), did not inhibit concAinduced MDC formation (Figure S2B). Together, these results suggest that MDCs are

190 not responsive to typical mitochondrial stressors, including loss of mitochondrial 191 membrane potential, oxidative stress, ATP depletion, or iron deprivation. containing high levels of amino acids (AA), but not in synthetic medium (Low AA), or in

195 minimal medium completely lacking amino acids (No AA) (Figure 2B). Steady-state amino acid analysis indicated that cells grown under the latter two conditions contained

197 lower intracellular amino acid pools when compared to cells grown in rich media (Figure 198 S2C). Supplementation of cells with casamino acids (Cas AA) restored MDC formation 199 in medium containing low or no amino acids (Figure 2B).

As an alternative mechanism to create acute, intracellular amino acid surplus, we

201 treated cells with cycloheximide (CHX), which blocks incorporation of amino acids into

202 proteins (Beugnet et al., 2003) (Figure 2A). Like concA, CHX also activated formation of

203 Tom70-positive, Tim50-negative structures in a high percentage of cells (Figure 2C).

204 These structures were cargo selective, dynamic, and approached sizes close to $1 \mu \mathrm{m}$ in 205 diameter (Figures 2C-D, S2D and Video S3). CHX-induced MDCs lacked mtDNA and 206 membrane potential (Figures S2E-F). Importantly, depletion of amino acids prevented 207 CHX-induced MDC formation, and supplementation with casamino acids restored 
MDCs, indicating that MDC induction by $\mathrm{CHX}$ was amino acid-dependent, and not simply due to inhibition of protein synthesis (Figure 2E). Thus, MDC formation is triggered by acute elevation of intracellular amino acids.

211

\section{BCAAs and their catabolites activate MDC formation}

213 Next, we sought to identify the specific amino acids that trigger MDC activation. We

214 found that addition of single amino acids to low amino acid medium restored MDC

215 formation in the presence of concA to different extents (Figure 3A). Leucine was the

216 most potent activator of MDC formation, followed by methionine, isoleucine, and

217 glutamate, while several other amino acids, including arginine and proline, did not

218 activate formation of MDCs (Figure 3A). In yeast, the most potent activators of MDC

219 formation, including the BCAAs leucine and isoleucine as well as methionine, are all

220 metabolized via the Ehrlich pathway, which converts amino acids to their corresponding

$221 \alpha$-keto acid via transamination, followed by decarboxylation to an aldehyde and

222 oxidation to an alcohol (Figure 3B) (Hazelwood et al., 2008). To test whether

223 downstream metabolic products of leucine catabolism also trigger MDC formation, we

224 compared MDC formation induced by leucine to its downstream catabolic products

225 ketoisocaproic acid (KIC), isovaleraldehye (IVA), and isoamylalcohol (IAA) (Figure 3C).

226 Addition of KIC and IVA to low amino acid medium restored MDC formation in the

227 presence of concA to the same extent or even more robustly than leucine, while IAA

228 had no effect (Figure 3C). Remarkably, $\mu \mathrm{M}$ concentrations of IVA alone, without

229 impairment of V-ATPase function or inhibition of protein synthesis, potently activated

230 MDC formation (Figure 3D-E). Like concA and CHX, IVA-activated MDCs were cargo 
231 selective, reached sizes of nearly $1 \mu \mathrm{m}$ in diameter, and lacked mtDNA and a

232 membrane potential (Figures 3E-F, S3A-B). Similar results were obtained with the

233 aldehyde derivative of methionine, which stimulated MDC formation both in the

234 presence and absence of lysosome impairment (Figures S3C-H). Altogether, these

235 results suggest that BCAAs and their catabolic derivatives are potent activators of MDC

236 formation.

237

238 Amino acids stimulate MDC formation independent of known nutrient sensing

239 pathways

240 In yeast, amino acid availability is transmitted through several nutrient sensing

241 pathways including the mechanistic Target of Rapamycin (mTOR) kinase, a central

242 component of two independent nutrient sensing complexes, mTORC1 and mTORC2

243 (Saxton and Sabatini, 2017) (Figure 4A), GCN2 (Hinnebusch, 2005), Gap1/Protein

244 Kinase A (PKA) (Donaton et al., 2003) and the Ssy1-Ptr3-Ssy5 (SPS) sensing pathway

245 (Ljungdahl, 2009). These pathways are activated by elevated nutrients and regulate

246 numerous cellular processes to coordinate cell growth with nutrient availability. Since

247 leucine is critical for mTOR signaling (Hara et al., 1998), we reasoned that increased

248 mTOR activity in response to amino acid elevation might provide the signal to activate

249 MDC formation (Figure 4A). To our surprise, inhibiting mTOR with rapamycin (Saxton

250 and Sabatini, 2017) did not block concA or CHX-induced MDC formation (Figure 4B). In

251 fact, rapamycin enhanced concA-induced MDC formation (Figure 4B), and activated

252 cargo selective MDC formation on its own with characteristics identical to concA and 
253 CHX-induced MDC formation (Figures 4C-D, S4A-C and Video S4). Similar results were

254 observed with Torin1 (Thoreen et al., 2009), another mTOR inhibitor (Figures S4D-E).

In yeast, inhibition of mTOR signaling, which occurs naturally during amino acid

256 starvation, enhances nutrient availability by blocking protein translation (Barbet et al.,

257 1996), stimulating expression of the general amino acid permease Gap1 (Cardenas et

258 al., 1999), and activating autophagy (Noda and Ohsumi, 1998). Therefore, inhibiting the

259 mTOR pathway in nutrient-replete conditions resulted in intracellular amino acid

260 overload, consistent with prior observations (Figure S4F) (Chen and Kaiser, 2003). As

261 with concA and $\mathrm{CHX}$, rapamycin and Torin1-dependent MDC induction was reduced in

262 low amino acid medium, blocked in amino acid free medium, and restored with

263 casamino acids (Figures 4E and S4G). These results demonstrate that high intracellular

264 amino acid concentrations trigger MDC formation through an mTOR-independent

265 mechanism, and that inhibiting mTOR induces MDCs by elevating intracellular amino

266 acids. In addition to the mTOR pathway, we also tested whether genetic alteration of

267 other common amino acid sensing pathways in yeast impacted MDC formation. We

268 found that deletion of GCN2, GLN3, GPA2, GPR1, and SSY1, which dismantle a variety

269 of nutrient signaling system in yeast, had no impact on MDC formation in response to

270 concA, CHX, or rapamycin (Figure 4F). Thus, amino acid surplus triggers MDC

271 formation independently of common nutrient sensing pathways.

272

273 MDCs regulate the abundance of the carrier receptor Tom70 on mitochondria

274 Through microscopy-based screening, we previously found that the cargo localized to

275 MDCs included Tom70, a mitochondrial OM receptor that facilitates import of the SLC25 
276 nutrient transporter family into mitochondria (Figure S5E) (Sollner et al., 1990),

277 members of the inner membrane-localized SLC25 family themselves, as well as a few

278 other OM proteins that are known to be Tom70 clients (Hughes et al., 2016). The

279 SLC25 protein family consists of over 30 members in yeast and 50-plus members in

280 mammals, and these multi-pass IM proteins are responsible for the exchange of nearly

281 all metabolites across the mitochondrial IM (Palmieri and Monne, 2016). Because

282 MDCs are nutrient responsive, we wondered whether these structures functioned to

283 modulate the Tom70 pathway in mitochondria by reducing Tom70 levels on the

284 mitochondrial surface upon nutrient surplus. Consistent with this idea, we found that

285 after rapamycin treatment, Tom70 was nearly 6-fold enriched in the MDC compared to

286 the mitochondrial tubule (Figure $5 A-B$ ). This enrichment led to a $\sim 50 \%$ reduction in the

287 abundance of Tom70 in the mitochondrial tubule after two hours (Figure 5C). Similar

288 decreases in mitochondrial tubule abundance of Tom70 were observed across all

289 known MDC inducers (Figure S5A). By contrast, mitochondrial levels of Tim50 and Ilv2,

290 IM and matrix proteins which do not associate with MDCs, were not reduced upon

291 treatment with MDC inducers (Figures S5B-D). Importantly, in an accompanying

292 manuscript, we found that the conserved mitochondrial localized GTPase Gem1

293 (Frederick et al., 2004) is required for MDC formation (English et al., 2020). Ablation of

294 MDC formation by deleting GEM1 prevented removal of Tom70 from mitochondria in

295 response to MDC activating treatments (Figures 5C and S5A), indicating that MDCs

296 diminish the abundance of Tom70 in mitochondria under elevated amino acids

297 conditions. 
In addition to the carrier import receptor Tom70, mitochondria also harbor

another OM import receptor, Tom20, which binds and facilitates import of matrix and IM

localized proteins that contain canonical N-terminal mitochondrial targeting sequences

301

(MTSs) (Figure S5E) (Abe et al., 2000; Moczko et al., 1994; Moczko et al., 1993). In

contrast to Tom70 and its clients, we previously found via microscopy screening that

Tom20 and all MTS containing proteins were excluded from MDCs (Hughes et al.,

2016). Using super resolution microscopy, we confirmed this observation here. We

found that while Tom20 is present at low levels in MDCs, it is not enriched above the

signal in the mitochondrial tubule, and is not depleted from the mitochondrial tubule

under any MDC inducing condition (Figures 5D-F and S5F). These results indicate that

MDCs specifically target the Tom70 pathway in response to elevated amino acids, while leaving the Tom20 pathway and its clients unchanged.

\section{MDCs are regulated by mitochondrial nutrient transporter abundance}

312 Given that MDCs sequester machinery and clients of the Tom70 carrier pathway in

313 response to elevated amino acids (Hughes et al., 2016), we reasoned that modulation

314 of SLC25 transporter levels may in turn regulate MDC formation. Consistent with this

315 idea, overexpression of Tom70, which provides more binding sites for carriers on the

316 mitochondrial surface, caused constitutive MDC formation in cells grown in high amino

317 acid medium, and stimulated MDC formation in concA-treated cells grown in low amino

318 acid medium, where MDC formation is normally not apparent (Figure 6A). Moreover,

319 deletion of TOM70 (Hines et al., 1990; Steger et al., 1990) alone or in combination with

320 its paralog TOM71 (Schlossmann et al., 1996), which prevents localization of carriers to 
321 mitochondria (Ryan et al., 1999), severely reduced MDC formation in response to all

322 known MDC inducers (Figure 6B). By contrast, MDC formation was largely unaffected in

323 cells overexpressing or deficient for TOM20 (Figures 6C-D), again highlighting the

324 specificity of the MDC pathway for Tom70.

To more directly analyze the impact of carrier abundance on the MDC pathway,

326 we overexpressed 28 individual members of the SLC25 family in wild-type cells, and

327 screened for carriers that impacted the magnitude of MDC formation (Figures S6A-B).

328 We identified numerous SLC25 carriers that constitutively activated MDC formation when overexpressed in cells grown in high amino acid medium, and stimulated MDC formation in concA-treated cells grown in low amino acid medium (Figures 6E-F and

331 S6A-B). Interestingly, several of these carriers play direct or indirect roles in amino acid

332 metabolism. By contrast, overexpression of the non-MDC IM client Tim50 did not

333 activate MDC formation (Figure S6C). Collectively, these results suggest that the MDC

334 pathway selectively targets the Tom70 carrier pathway in response to amino acid

335 alterations, and that modulating mitochondrial carrier levels via direct carrier

336 overexpression, or by regulating the abundance of the carrier receptor Tom70 governs

337 the extent of MDC formation within cells.

341 Finally, we wanted to determine whether MDCs play a role in regulating cellular health

342 under conditions of elevated amino acid stress. We first tested whether deletion of

343 GEM1, which is required for MDC formation (English et al., 2020), impacted cell growth 
under MDC inducing conditions. Serial-dilution growth assays demonstrated mild to no growth defects in gem1 $1 \Delta$ strains when grown in the presence of concA combined with

346 leucine or methionine addition-conditions that activate the MDC pathway (Figure 7A).

347 Since gem $1 \Delta$ strains did not exhibit robust growth phenotypes under MDC inducing

348 conditions, we considered the existence of redundant pathways that protect cells from

349 amino acid stress in absence of MDC formation. A strong candidate for such a system

350 is the endosomal sorting complexes required for transport (ESCRT)-dependent MVB

351 pathway. This pathway post-translationally regulates levels of nutrient transporters at

352 the plasma membrane (PM) via ubiquitin-mediated receptor internalization and

353 destruction in the lysosome, and mutants in this system exhibit elevated amino acid

354 uptake from the environment (Katzmann et al., 2002; Rubio-Texeira and Kaiser, 2006).

$355 \quad$ Prior studies showed that the ESCRT/MVB system downregulates PM

356 transporters in response to rapamycin and $\mathrm{CHX}$ - two conditions that also activate MDC

357 formation (Hatakeyama and De Virgilio, 2019; Lin et al., 2008; Nikko and Pelham,

358 2009). Utilizing the fluorescently labelled branched-chain amino acid permease Bap2-

359 GFP (Grauslund et al., 1995), we confirmed via microscopy and western blot that

360 treatment with rapamycin or $\mathrm{CHX}$ triggered a reduction in Bap2-GFP levels at the PM,

361 and a corresponding increase in Bap2-GFP processing in the lysosome-the latter

362 indicated by an accumulation of free GFP that is proteolytically released from Bap2-

363 GFP in the lysosome (Figures 7B-C). Remarkably, Bap2 internalization and processing

364 also occurred in other conditions that activate MDCs, including concA, or treatment with

365 the leucine and methionine derived aldehydes IVA and MTPA (Figures 7B-C). We

366 confirmed that concA-induced Bap2 internalization and degradation was prevented in 
367 cells lacking components of the ESCRT machinery (Hurley and Emr, 2006), including 368 VPS27 and DID4 (Figures 7C-D and S7A-B). Thus, under conditions of amino acid excess, cells simultaneously activate MDC formation and reduce levels of PM nutrient

370 transporters via the ESCRT-dependent MVB pathway.

371 Based on these observations, we reasoned that the ESCRT-dependent MVB

372 pathway and MDCs may act in parallel to protect cells from amino acid stress.

373 Consistent with this idea, MDC formation was elevated in cells lacking the ESCRT

374 components VPS27 and DID4 (Figures 7E and S7C). Moreover, while cells lacking 375 either GEM1 or components of the ESCRT machinery exhibited mild to no growth 376 defects in the presence of high leucine or methionine, simultaneous loss of both the

377 MDC and the ESCRT-dependent MVB pathways was detrimental under high amino acid 378 conditions, and severely inhibited growth when combined with loss of lysosome function 379 (Figures 7F and S7D-E). Importantly, growth inhibition only occurred in response to the 380 MDC inducing amino acids leucine and methionine, and was not caused by arginine or 381 proline, two amino acids that do not activate MDC formation (Figures 7F and S7D-E). 382 Altogether, these results suggest that MDCs act in parallel with lysosomes and the 383 ESCRT-dependent MVB pathway to ensure cellular survival under conditions of amino 384 acid stress.

\section{DISCUSSION}

387 Here, we demonstrate that a previously identified foci associated with mitochondria, the 388 MDC (Hughes et al., 2016), is a dynamic, lumen-containing organelle that is generated 389 from mitochondria in response to intracellular amino acid overload. Upon formation, 
MDCs selectively sequester the machinery and cargo of the Tom70 metabolite carrier import pathway away from the rest of the mitochondrial network. Based on our data, we speculate that MDCs function to protect cells from amino acid stress, potentially by acting as part of a broad cellular network that coordinately regulates intracellular nutrient distribution. The three nodes of this network, as depicted in Figure 7G, are the

395 ESCRT-dependent MVB pathway that controls metabolite transporters at the PM

396 (Katzmann et al., 2002), the lysosome, which sequesters and stores amino acids in its 397 lumen (Klionsky et al., 1990; Wiemken and Dürr, 1974), and the MDC, which regulates 398 the metabolite carrier pathway on mitochondria. Consistent with the idea that these systems act in parallel to preserve intracellular amino acid homeostasis, combined loss

400 of the MDC pathway, the ESCRT-dependent MVB system, and lysosome amino acid 401 storage leads to cellular toxicity that is amino acid dependent. These results highlight

402 the importance of maintaining proper amino acid homeostasis in cells, and suggest cells 403 contain dedicated systems to mitigate toxicity associated with improper levels of amino 404 acids.

405 The discovery of a new nutrient-sensitive compartment raises many important 406 questions for future exploration. Central among them is how MDCs prevent toxicity from 407 excess BCAAs and methionine? Our data indicate that MDCs negatively regulate the 408 levels of Tom70, an OMM protein required for import of nutrient carriers into 409 mitochondria, and that the MDC pathway is in turn sensitive to carrier expression levels. 410 Thus, we speculate that much like the MVB-dependent ESCRT pathway that regulates

411 transporter levels on the PM, the MDC in turn modulates metabolite carrier levels on the 412 mitochondria in response to changes in cellular nutrient supply. A key difference 
413 between the PM and mitochondrial systems is the fact that mitochondrial nutrient

414 carriers are localized on the mitochondrial IM, which is not accessible from the

415 cytoplasm. Thus, sequestering the import receptor on the mitochondrial surface,

416 Tom70, may enable cells to indirectly control mitochondrial nutrient transporters.

417 Additional studies are required to solidify whether the MDC incorporates both the

418 mitochondrial OM and IM, and whether carrier proteins localized to the MDC are

419 enriched from the IM.

420 Regardless of how the MDC protects cells from amino acid stress, our data

421 strongly suggests a role for mitochondria in mediating the toxic effects of excess BCAAs

422 and methionine. We recently discovered that defects in cysteine homeostasis cause

423 cellular toxicity by altering iron availability and mitochondrial respiration (Hughes et al.,

424 2020). However, MDCs do not respond to cysteine or iron perturbations, indicating that

425 the mechanism by which excess BCAAs and methionine become toxic to cells are

426 distinct from cysteine's impact on mitochondrial respiration. Interestingly, elevated

427 BCAAs have been linked to the development of metabolic disorders including Type II

428 diabetes (Knebel et al., 2016; Newgard et al., 2009; Wang et al., 2011; Xu et al., 2013).

429 Current speculation suggests that elevated BCAAs or their catabolites may alter insulin

430 production by impairing mitochondrial function (Lynch and Adams, 2014). However, the

431 mechanism by which this occurs remains unknown. Deciphering the mode of action of

432 BCAA and methionine toxicity is an important area of future research, as well as

433 understanding how MDCs function to protect cells from the toxic effects of these amino

434 acids. 
We show that MDCs are responsive to elevated levels of intracellular amino

436

437

438

439

440

441

442

444

445

446

447 stimulate MDC production also trigger downregulation of nutrient transporters via the

448 MVB pathway (Figures 7B-C). Thus, it seems likely that these two pathways utilize

449 similar nutrient sensing systems - the identify of which is unknown at this time. Moving

450 forward, it will be important to determine how the MDC and MVB systems act

451

452

453

\section{ACKNOWLEDGEMENTS}

455 We thank members of the A.L.H. and J.M.S laboratories for discussion and manuscript

456 comments and L. VanderMeer (Utah) and P. Guo (Utah) for technical assistance.

457 Metabolomics analysis was performed at the University of Utah Metabolomics Core 
458 directed by J. Cox and supported by National Institutes of Health (NIH) grants

459 1S10OD016232-01, 1S10OD021505-01 and 1U54DK110858-01. Research was

460 supported by NIH grants AG043095, GM119694, AG061376, and AG055648 (A.L.H.),

461 NIH T32GM007464 (A.M.E.), NIH GM53466 and GM84970 (J.M.S.), AHA

462 18PRE33960427 (M.H.S.) and the Howard Hughes Medical Institute (J.M.S.). A.L.H.

463 was further supported by an American Federation for Aging Research Junior Research

464 Grant, United Mitochondrial Disease Foundation Early Career Research Grant, Searle

465 Scholars Award, and Glenn Foundation for Medical Research Award.

466

467 AUTHOR CONTRIBUTIONS

468 Conceptualization, M.H.S., A.M.E., A.L.H., and J.M.S.; Methodology, M.H.S., A.M.E., 469 A.L.H., and J.M.S.; Formal Analysis, M.H.S., A.M.E., and T.J.C.; Investigation, M.H.S., 470 A.M.E., and T.J.C.; Writing - Original Draft, A.L.H., A.M.E., and M.H.S.; Writing -

471 Reviewing and Editing, A.L.H. and J.M.S.; Visualization, M.H.S. and A.M.E.;

472 Supervision, A.L.H. and J.M.S.; Funding Acquisition, A.L.H., J.M.S., A.M.E., and M.H.S.

473

474 DECLARATION OF INTERESTS

475 The authors declare no competing interests.

477 MAIN FIGURE LEGENDS

478 Figure 1. MDCs are Dynamic, Mitochondria-Associated Structures with Distinct 479 Lumens 
480 (A) Widefield images (left) and line-scan analysis (right) of concanamycin A (ConcA)-

481 induced MDC formation in yeast cells expressing Tom70-GFP and Tim50-mCherry.

482 White arrow marks MDC. White line marks fluorescence intensity profile position. Line-

483 scan $Y$ axis corresponds to Tom70-GFP and Tim50-mCherry fluorescence intensity.

484 Black arrow marks mitochondrial tubule. Bracket marks MDC. Scale bar $=2 \mu \mathrm{m}$.

485 (B) Super-resolution images (left) and line-scan analysis (right) of ConcA-induced MDC 486 formation in yeast cells expressing Tom70-GFP and Tim50-mCherry. White arrow

487 marks MDC. White line marks fluorescence intensity profile position. Left and right line488 scan $\mathrm{Y}$ axis correspond to Tom70-GFP and Tim50-mCherry fluorescence intensity, 489 respectively. Black arrow marks mitochondrial tubule. Bracket marks MDC. Scale bar = $4902 \mu \mathrm{m}$.

491 (C) Scatter plot showing the diameter of ConcA-induced MDCs. Error bars show mean $492(0.7282 \mu \mathrm{m}) \pm \mathrm{SD}$ of $n=30$ MDCs.

493 (D) Z-series corresponding to yeast cell treated with ConcA in (B). Image intervals are $4940.15 \mu \mathrm{m}$. Scale bar $=2 \mu \mathrm{m}$.

495 (E) Super-resolution images of ConcA-treated yeast cells expressing Tom70-GFP, 496 stained with DAPI to label mitochondrial DNA. White arrow marks MDC. Scale bar = $4972 \mu \mathrm{m}$.

498 (F) Super-resolution images of ConcA treated yeast cells expressing Tom70-GFP 499 stained with fluorescent membrane potential indicator TMRM. White arrow marks MDC. 500 Scale bar $=2 \mu \mathrm{m}$. 
501 (G) Time-lapse images of ConcA-induced MDC formation in yeast cells expressing

502 Tom70-GFP. Images were acquired over 120 minutes (min). Arrowhead marks MDC.

503 Scale bar $=2 \mu \mathrm{m}$. See also Video S1.

504 See also Figure S1.

505

506 Figure 2. MDC Formation is Triggered in Response to Amino Acid Elevation

507 (A) Model of intracellular amino acid (AA) homeostasis. AAs are metabolized, utilized

508 for protein biosynthesis or stored in the yeast vacuole (lysosome). Defects in these

509 processes cause buildup of amino acids in the cytoplasm.

510 (B) Quantification of concanamycin A (ConcA)-induced MDC formation in media

511 containing high, low or no amino acids (AA). Cas AA indicates where bulk amino acids

512 were added back to media in the form of hydrolyzed casein. Error bars show mean \pm SE

513 of three replicates with $n=100$ cells per replicate.

514 (C) Super-resolution images (left) and line-scan analysis (right) of cycloheximide (CHX)-

515 induced MDC formation in yeast cells expressing Tom70-GFP and Tim50-mCherry.

516 White arrow marks MDC. White line marks fluorescence intensity profile position. Left

517 and right $Y$ line-scan axis correspond to Tom70-GFP and Tim50-mCherry fluorescence

518 intensity, respectively. Black arrow marks mitochondrial tubule. Bracket marks MDC.

519 Scale bar $=2 \mu \mathrm{m}$.

520 (D) Scatter plot showing the diameter of $\mathrm{CHX}$-induced MDCs. Error bars show mean

$521 \quad(0.8095 \mu \mathrm{m}) \pm \mathrm{SD}$ of $n=30$ MDCs.

522 (E) Quantification of CHX-induced MDC formation in media containing high, low or no

523 amino acids (AA). Cas AA indicates where bulk amino acids were added back to media 
524 in the form of hydrolyzed casein. Error bars show mean \pm SE of three replicates with $n=$

525100 cells per replicate.

526 See also Figure S2.

527

528 Figure 3. Branched-Chain Amino Acids and Their Derivatives Activate MDC

529 Formation

530 (A) Quantification of concanamycin A (ConcA)-induced MDC formation in high or low

531 amino acid medium supplemented with the indicated amino acid (AA). Error bars show

532 mean \pm SE of three replicates with $n=100$ cells per replicate.

533 (B) Diagram of branched chain amino acid catabolism via the Ehrlich Pathway.

534 (C) Quantification of ConcA-induced MDC formation in low amino acid medium

535 supplemented with leucine (Leu) or its catabolites ketoisocaproic acid (KIC),

536 isovaleraldehyde (IVA) or isoamylalcohol (IAA). Error bars show mean \pm SE of three

537 replicates with $n=100$ cells per replicate.

538 (D) Quantification of MDC formation in high amino acid medium supplemented with Leu,

$539 \mathrm{KIC}$, IVA or IAA in the absence of drug treatment. Error bars show mean \pm SE of three

540 replicates with $n=100$ cells per replicate.

541 (E) Super-resolution images (left) and line-scan analysis (right) of IVA-induced MDC

542 formation in yeast cells expressing Tom70-GFP and Tim50-mCherry. White arrow

543 marks MDC. White line marks fluorescence intensity profile position. Left and right line-

544 scan $\mathrm{Y}$ axis correspond to Tom70-GFP and Tim50-mCherry fluorescence intensity,

545 respectively. Black arrow marks mitochondrial tubule. Bracket marks MDC. Scale bar =

$5462 \mu \mathrm{m}$. 
547 (F) Scatter plot showing the diameter of IVA-induced MDCs. Error bars show mean

$(0.938 \mu \mathrm{m}) \pm \mathrm{SD}$ of $n=50 \mathrm{MDCs}$

See also Figure S3.

550

551 Figure 4. Amino Acids Activate MDC Formation Independent of Known Nutrient

\section{Sensing Pathways}

553 (A) Schematic of amino acid (AA)-induced activation of mTOR signaling.

554 (B) Quantification of concanamycin A (ConcA) and cycloheximide (CHX)-induced MDC

555 formation in the absence or presence of rapamycin (Rap). Error bars show mean $\pm \mathrm{SE}$

556 of three replicates with $n=100$ cells per replicate.

557 (C) Super-resolution images (left) and line-scan analysis (right) of Rap-induced MDC

558 formation in yeast cells expressing Tom70-GFP and Tim50-mCherry. White arrow

559 marks MDC. White line marks fluorescence intensity profile position. For DMSO, left

560 and right line-scan $Y$ axis correspond to Tom70-GFP and Tim50-mCherry fluorescence

561 intensity, respectively. For Rap, left line-scan Y axis corresponds to both Tom70-GFP

562 and Tim50-mCherry fluorescence intensity. Black arrow marks mitochondrial tubule.

563 Bracket marks MDC. Scale bar $=2 \mu \mathrm{m}$.

564 (D) Scatter plot showing the diameter of Rap-induced MDCs. Error bars show mean

$(0.8829 \mu \mathrm{m}) \pm \mathrm{SD}$ of $n=30 \mathrm{MDCs}$.

566 (E) Quantification of Rap-induced MDC formation in media containing high, low or no

567 amino acids (AA). Cas AA indicates where bulk amino acids were added back to media

568 in the form of hydrolyzed casein. Error bars show mean \pm SE of three replicates with $n=$

569100 cells per replicate. 
570 (F) Quantification of ConcA, CHX and Rap-induced MDC formation in yeast strains

571 deficient for the indicated nutrient sensing pathway. Error bars show mean \pm SE of three

572 replicates with $n=100$ cells per replicate.

573 See also Figure S4.

574

575 Figure 5. MDCs Selectively Sequester the Carrier Receptor Tom70 from

576 Mitochondria

577 (A) Super-resolution images (left) and line-scan analysis (right) of rapamycin (Rap)-

578 induced MDC formation in yeast cells expressing Tom70-GFP. Right image (Tom70*)

579 shows where the fluorescence intensity has been increased post imaging to visualize

580 the mitochondrial tubule. White line marks fluorescence intensity profile position. Left

581 line-scan Y axis corresponds to the Tom70-GFP fluorescence intensity. Black arrow

582 marks mitochondrial tubule. Bracket marks MDC. Scale bar $=2 \mu \mathrm{m}$.

583 (B) Scatter plot showing the normalized Tom70-GFP fluorescence intensity in Rap-

584 induced MDCs compared to the adjacent mitochondrial tubule. Error bars show mean \pm

585 SD of $n=45$ cells from three per replicates with $n=15$ cells per replicate.

586 (C) Normalized mitochondrial Tom70-GFP fluorescence in wild-type (WT) and gem1د

587 cells treated with Rap compared to DMSO. Error bars show mean \pm SE of three

588 replicates with $n=15$ cells per replicate.

589 (D) Super-resolution images (left) and line-scan analysis (right) of Rap-induced MDC

590 formation in yeast cells expressing Tom20-GFP and Tom70-mCherry. White arrow

591 marks MDC. White line marks fluorescence intensity profile position. Line-scan Y axis 
592 corresponds to Tom20-GFP and Tom70-mCherry fluorescence intensity. Black arrow

593 marks mitochondrial tubule. Bracket marks MDC. Scale bar $=2 \mu \mathrm{m}$.

594 (E) Scatter plot showing the normalized Tom20-GFP fluorescence intensity in Rap-

595 induced MDCs compared to the adjacent mitochondrial tubule. Error bars show mean \pm

596 SD of $n=45$ cells from three per replicates with $n=15$ cells per replicate.

597 (F) Normalized mitochondrial Tom20-GFP fluorescence in wild-type (WT) and gem1

598 cells treated with Rap or DMSO. Error bars show mean \pm SE of three replicates with $n=$

59915 cells per replicate.

600 See also Figure S5.

601

602 Figure 6. Mitochondrial Carrier Levels Control MDC Formation

603 (A) Quantification of concanamycin A (ConcA)-induced MDC formation in TOM70

604 overexpressing or empty vector (EV) control cells in high and low amino acid media.

605 Error bars show mean \pm SE of three replicates with $n=100$ cells per replicate.

606 (B) Quantification of ConcA, cycloheximide (CHX), rapamycin (Rap), isovaleraldehyde

607 (IVA) and 3-methylthiopropanal (MTPA)-induced MDC formation in wild-type (WT),

608 tom70 $\Delta$ and tom70 $\Delta$ tom $71 \Delta$ cells. Error bars show mean \pm SE of three replicates with $n$

$609=100$ cells per replicate

610 (C) Quantification of ConcA-induced MDC formation in TOM20 overexpressing or EV

611 control cells in high and low amino acid media. Error bars show mean \pm SE of three

612 replicates with $n=100$ cells per replicate. 
613 (D) Quantification of ConcA, CHX, Rap, IVA and MTPA-induced MDC formation in WT

614 and tom $20 \Delta$ cells. Error bars show mean \pm SE of three replicates with $n=100$ cells per

615 replicate.

616 (E) Quantification of MDC formation in cells expressing the indicated mitochondrial

617 carrier in high amino acid media in absence of drug treatment. Error bars show mean \pm

618 SE of three replicates with $n=100$ cells per replicate.

619 (E) Quantification of MDC formation in cells expressing the indicated mitochondrial

620 carrier in low amino acid media in presence of ConcA. Error bars show mean \pm SE of

621 three replicates with $n=100$ cells per replicate.

622 See also Figure S6.

623

624 Figure 7. MDCs Cooperate with MVBs and Lysosomes to Protect Cells from Toxic

625 Levels of Amino Acids

626 (A) Growth of wild-type and gem1 $\Delta$ cells in presence and absence of concanamycin A

627 (ConcA) on low amino acid media supplemented with $10 \mathrm{mg} / \mathrm{ml}$ leucine or methionine.

628 (B) Widefield images showing internalization of Bap2-GFP upon rapamycin (Rap),

629 cycloheximide (CHX), ConcA, isovaleraldehyde (IVA) or 3-methylthiopropanal (MTPA)

630 treatment. Scale bar $=2 \mu \mathrm{m}$.

631 (C) Western blot analysis of Bap2-GFP clipping upon treatment with Rap, CHX, ConcA,

632 IVA and MTPA in wild-type and vps27 $\Delta$ cells. Pgk1 - loading control.

633 (D) Widefield images showing stabilization of Bap2-GFP on the plasma membrane in

634 Rap, CHX, ConcA, IVA or MTPA treated vps27 $\Delta$ cells. Bright structures resemble

635 typical class E compartments found in ESCRT mutants. Scale bar $=2 \mu \mathrm{m}$. 
636 (E) Quantification of ConcA-induced MDC formation in low amino acid media

637 supplemented with the indicated amount of leucine in wild-type (WT) and vps27 $\Delta$ cells.

638 Error bars show mean \pm SE of three replicates with $n=100$ cells per replicate.

639 (F) Growth of wild-type, gem1 1 , vps27 $\Delta$ and gem1 $\Delta$ vps27 $\Delta$ strains in presence and

640 absence of ConcA on low amino acid media supplemented with $10 \mathrm{mg} / \mathrm{ml}$ leucine or

641 arginine.

642 (G) Yeast cells utilize multiple systems, including lysosomal amino acid

643 compartmentation, ESCRT-dependent control of plasma-membrane localized amino

644 acid transporter via the MVB pathway and the MDC pathway to counteract toxic levels

645 of amino acids. Simultaneous defects in all three systems render cells sensitive to toxic

646 levels of amino acids.

647 See also Figure S7.

648

649 Figure S1. V-ATPase Inhibition Induces Dynamic MDC Formation, Related to

650 Figure 1

651 (A) Western blot showing time course of auxin-induced Vma2-AID*-FLAG depletion.

652 (B) Time course of auxin-induced inhibition of vacuole acidification due to Vma2-AID*-

653 FLAG depletion assayed by staining with pH-dependent dye quinacrine. Error bars

654 show mean \pm SE of three replicates, $n=30$ cells per replicate.

655 (C) Quantification of Vma2-AID*-FLAG-depletion induced MDC formation over time.

656 Error bars show mean \pm SE of three replicates with $n=100$ cells per replicate. 
657 (D) Widefield images of auxin-induced MDC formation in the indicated yeast strains

658 expressing Tom70-GFP and Tim50-mCherry. White arrow marks MDC. Scale bar $=2$

$659 \mu \mathrm{m}$.

660 (E) Time-lapse images of ConcA-induced MDC formation in yeast cells expressing

661 Tom70-GFP. Images were acquired over 120 minutes (min). Arrowhead marks MDC.

662 Scale bar $=2 \mu \mathrm{m}$. See also Video S2.

663

664 Figure S2. Additional Characteristics of MDCs, Related to Figure 2

665 (A) Quantification of MDC formation in yeast cells treated with concanamycin A

666 (ConcA), oligomycin (Oligo), antimycin A (AntA), carbonyl cyanide 4-

667 (trifluoromethoxy)phenylhydrazone (FCCP), $\mathrm{CoCl}_{2}$, tunicamycin (Tunica) or $\mathrm{H}_{2} \mathrm{O}_{2}$. Error

668 bars show mean \pm SE of three replicates with $n=100$ cells per replicate

669 (B) Quantification of MDC formation in yeast cells treated with ConcA or

670 bathophenanthroline disulfonate (BPS) in high amino acid media in presence or

671 absence of exogenous iron. Error bars show mean \pm SE of three replicates with $n=100$

672 cells per replicate.

673 (C) Relative amino acid levels in yeast cells grown in media containing low or no amino

674 acids compared to high amino acid medium assayed by GC-MS. Error bars show mean

$675 \pm$ SE of three replicates.

676 (D) Time-lapse images of $\mathrm{CHX}$-induced MDC formation in yeast cells expressing

677 Tom70-GFP. Images were acquired over 120 minutes (min). Arrowhead marks MDC.

678 Scale bar $=2 \mu \mathrm{m}$. See also Video S3. 
679 (E) Super-resolution images of cycloheximide $(\mathrm{CHX})$ treated yeast cells expressing

680 Tom70-GFP stained with DAPI to label mitochondrial DNA. White arrow marks MDC.

681 Scale bar $=2 \mu \mathrm{m}$.

682 (F) Super-resolution images of $\mathrm{CHX}$ treated yeast cells expressing Tom70-GFP stained 683 with fluorescent membrane potential indicator TMRM. White arrow marks MDC. Scale 684 bar $=2 \mu \mathrm{m}$.

685

686 Figure S3. Leucine and Methionine Derivatives Activate MDC Formation, Related 687 to Figure 3

688 (A) Super-resolution images of isovaleraldehyde (IVA) treated yeast cells expressing 689 Tom70-GFP, stained with DAPI to label mitochondrial DNA. White arrow marks MDC. 690 Scale bar $=2 \mu \mathrm{m}$.

691 (B) Super-resolution images of IVA treated yeast cells expressing Tom70-GFP stained 692 with fluorescent membrane potential indicator TMRM. White arrow marks MDC. Scale 693 bar $=2 \mu \mathrm{m}$.

694 (C) Quantification of concanamycin A (ConcA)-induced MDC formation in low amino 695 acid medium supplemented with methionine (Met) or its catabolites a-ketomethiobutyric 696 acid (KMTB), 3-methylthiopropanal (MTPA) or 3-methylthiopropanol (MTP). Error bars 697 show mean \pm SE of three replicates with $n=100$ cells per replicate.

698 (D) Quantification of MDC formation in high amino acid medium supplemented with Met, $699 \mathrm{KMTB}, \mathrm{MTPA}$ or MTP in the absence of drug treatment. Error bars show mean \pm SE of 700 three replicates with $n=100$ cells per replicate. 
701 (E) Super-resolution images (left) and line-scan analysis (right) of MTPA-induced MDC

702 formation in yeast cells expressing Tom70-GFP and Tim50-mCherry. White arrow

703 marks MDC. White line marks fluorescence intensity profile position. Left and right line-

704 scan $Y$ axis correspond to Tom70-GFP and Tim50-mCherry fluorescence intensity,

705 respectively. Black arrow marks mitochondrial tubule. Bracket marks MDC. Scale bar =

$7062 \mu \mathrm{m}$.

707 (F) Scatter plot showing the diameter of MTPA-induced MDCs. Error bars show mean

$708 \quad(0.9587 \mu \mathrm{m}) \pm \mathrm{SD}$ of $n=50 \mathrm{MDCs}$

709 (G) Super-resolution images of MTPA-induced MDC formation in yeast cells expressing

710 Tom70-GFP, stained with DAPI to visualize mitochondrial DNA. White arrow marks

711 MDC. Scale bar $=2 \mu \mathrm{m}$.

$712(\mathrm{H})$ Super-resolution images of MTPA-induced MDC formation in yeast cells expressing

713 Tom70-GFP, stained with fluorescent membrane potential indicator TMRM. White arrow

714 marks MDC. Scale bar $=2 \mu \mathrm{m}$.

715

716 Figure S4. Rapamycin and Torin1 Treatment Activate MDC Formation, Related to

717 Figure 4

718 (A) Super-resolution images of Rap-induced MDC formation in yeast cells expressing

719 Tom70-GFP, stained with DAPI to visualize mitochondrial DNA. White arrow marks

720 MDC. Scale bar $=2 \mu \mathrm{m}$.

721 (B) Super-resolution images of Rap-induced MDC formation in yeast cells expressing

722 Tom70-GFP, stained with fluorescent membrane potential indicator TMRM. White arrow

723 marks MDC. Scale bar $=2 \mu \mathrm{m}$. 
724 (C) Time-lapse images showing Rap-induced MDC formation in yeast cells expressing

725 Tom70-GFP. Images were acquired over 119 minutes (min). Arrowhead marks MDC.

726 Scale bar $=2 \mu \mathrm{m}$. See also Video S4.

727 (D) Super-resolution images (left) and line-scan analysis (right) of Torin1-induced MDC

728 formation in yeast cells expressing Tom70-GFP and Tim50-mCherry. White arrow

729 marks MDC. White line marks fluorescence intensity profile position. Left and right line-

730 scan $Y$ axis correspond to Tom70-GFP and Tim50-mCherry fluorescence intensity,

731 respectively. Black arrow marks mitochondrial tubule. Bracket marks MDC. Scale bar =

$7322 \mu \mathrm{m}$.

733 (E) Scatter plot showing the diameter of Torin1-induced MDCs. Error bars show mean

$734(0.9156 \mu \mathrm{m}) \pm \mathrm{SD}$ of $n=50$ MDCs.

735 (F) Relative amino acid levels in Rap treated yeast cells grown in medium containing

736 high levels amino acids assayed by GC-MS. Error bars show mean \pm SE of three

737 replicates.

738 (G) Quantification of Torin1-induced MDC formation in media containing high, low or no

739 amino acids (AA). Cas AA indicates where bulk amino acids were added back to media

740 in the form of hydrolyzed casein. Error bars show mean \pm SE of three replicates with $n=$

741100 cells per replicate.

742

743 Figure S5. MDCs Selectively Remove Tom70, Related to Figure 5

744 (A) Normalized mitochondrial Tom70-GFP fluorescence in wild-type (WT) and gem1

745 cells treated with concanamycin A (ConcA), cycloheximide ( $\mathrm{CHX})$, isovaleraldehyde

746 (IVA) or 3-methylthiopropanal (MTPA)-compared to DMSO. Error bars show mean \pm SE 
747 of three replicates with $n=15$ cells per replicate. This graph shows additional data

748 generated in the experiments used for Figure 5C. The DMSO control is duplicated from

749 Figure 5C for comparison purposes.

750 (B) Normalized mitochondrial Tim50-GFP fluorescence in cells treated with ConcA,

$751 \mathrm{CHX}$, rapamycin (Rap), IVA or MTPA compared to DMSO. Error bars show mean \pm SE

752 of three replicates with $n=15$ cells per replicate.

753 (C) Super-resolution images of Rap-induced MDC formation in yeast cells expressing

754 Ilv2-GFP and Tom70-mCherry. White arrow marks MDC. Scale bar $=2 \mu \mathrm{m}$.

755 (D) Normalized mitochondrial Ilv2-GFP fluorescence in cells treated with ConcA, CHX,

756 Rap, IVA or MTPA compared to DMSO. Error bars show mean \pm SE of three replicates

757 with $n=15$ cells per replicate.

758 (E) Model of mitochondrial protein import. Tom70 promotes carrier biogenesis, whereas

759 Tom20 is required for protein import of pre-sequence containing proteins into

760 mitochondria.

761 (F) Normalized mitochondrial Tom20-GFP fluorescence in cells treated with ConcA,

$762 \mathrm{CHX}$, IVA or MTPA compared to DMSO. Error bars show mean \pm SE of three replicates

763 with $n=15$ cells per replicate. This graph shows additional data generated in the

764 experiments used for Figure 5F. The DMSO control is duplicated from Figure 5F for

765 comparison purposes.

766

767 Figure S6. Elevated SLC25 Carrier Expression Activates MDC Formation, Related

768 to Figure 6 
769 (A) Quantification of MDC formation in cells expressing the indicated mitochondrial

770 carrier in high amino acid media in absence of drug treatment. $n=100$ cells.

771 (B) Quantification of MDC formation in cells expressing the indicated mitochondrial

772 carrier in low amino acid media in presence of ConcA. $n=100$ cells.

773 (C) Quantification of ConcA-induced MDC formation in TIM50 overexpressing or empty

774 vector (EV) control cells in high and low amino acid media. Error bars show mean \pm SE

775 of three replicates with $n=100$ cells per replicate.

776

777 Figure S7. MDCs Cooperate with MVBs to Protect Cells from Amino Acid Toxicity,

$778 \quad$ Related to Figure 7

779 (A) Widefield images showing stabilization of Bap2-GFP on the plasma membrane in 780 rapamycin (Rap), cycloheximide ( $\mathrm{CHX}$ ), concanamycin $\mathrm{A}$ (ConcA), isovaleraldehyde

781 (IVA) or 3-methylthiopropanal (MTPA) treated did4A cells. Bright structures resemble

782 typical class E compartments found in ESCRT mutants. Scale bar $=2 \mu \mathrm{m}$.

783 (B) Western blot analysis of Bap2-GFP clipping upon treatment with Rap, CHX, ConcA,

784 IVA and MTPA in wild-type and did4A cells. Pgk1 - loading control.

785 (C) Quantification of ConcA-induced MDC formation in low amino acid media

786 supplemented with the indicated amount of leucine in wild-type (WT) and did4A cells.

787 Error bars show mean \pm SE of three replicates with $n=100$ cells per replicate.

788 (D) Growth of wild-type, gem1 1 , vps27 $\Delta$ and gem1 1 vps $27 \Delta$ strains in presence and 789 absence of ConcA on low amino acid media supplemented with $10 \mathrm{mg} / \mathrm{ml}$ methionine or 790 proline. 
791 (E) Growth of wild-type, gem1 1 , did4 $\Delta$ and gem1 $\Delta$ did4 $\Delta$ strains in presence and

792 absence of ConcA on low amino acid media supplemented with $10 \mathrm{mg} / \mathrm{ml}$ leucine,

793 methionine, proline or arginine.

794

795 SUPPLEMENTAL ITEM LEGENDS

796

797 Video S1. Concanamycin A-Induced MDC Formation, Related to Figure 1

798 An example of concanamycin A-induced MDC formation (from Figure 1G). Maximum

799 intensity projection video of yeast expressing Tom70-GFP treated with concanamycin A.

800 Images were taken every minute and are shown at 2 frames per second.

801

802 Video S2. Dynamic MDC Behavior, Related to Figure 1

803 An example of dynamic MDC behavior (from Figure S1E). Maximum intensity projection

804 video of yeast expressing Tom70-GFP treated with concanamycin A. Images were

805 taken every minute and are shown at 2 frames per second.

806

807 Video S3. Cycloheximide-Induced MDC Formation, Related to Figure 2

808 An example of cycloheximide-induced MDC formation (from Figure S2D). Maximum

809 intensity projection video of yeast expressing Tom70-GFP treated with cycloheximide.

810 Images were taken every minute and are shown at 2 frames per second.

811

812 Video S4. Rapamycin-Induced MDC Formation, Related to Figure 4 
813 An example of rapamycin-induced MDC formation (from Figure S4C). Maximum

814 intensity projection video of yeast expressing Tom70-GFP treated with rapamycin.

815 Images were taken every minute and are shown at 2 frames per second.

816

817 Table S1. Oligonucleotides Used in This Study, Related to Star Methods

818

\section{CONTACT FOR REAGENT AND RESOURCE SHARING}

820 Further information and requests for resources and reagents should be directed to and

821 will be fulfilled by the Lead Contact, Adam Hughes (hughes@biochem.utah.edu). All

822 unique/stable reagents generated in this study are available from the Lead Contact

823 without restrictions.

825 METHOD DETAILS

826

\section{Yeast Strains}

828 All yeast strains are derivatives of Saccharomyces cerevisiae S288c (BY) (Brachmann

829 et al., 1998). Strains expressing fluorescently tagged TOM70, TIM50, TOM20, ILV2,

830 TCD2, BAP2 and/or AID*-6xFLAG/6xFLAG tagged VMA2 from their native loci were

831 created by one step PCR-mediated C-terminal endogenous epitope tagging using

832 standard techniques and oligo pairs listed in Table S1. Plasmid templates for

833 fluorescent epitope tagging were from the pKT series of vectors (Sheff and Thorn,

834 2004). Plasmids used for AID*-6xFLAG/6xFLAG tagging and integration of GPD-OsTir1

835 into the LEU2 locus are described below. Correct integrations were confirmed by a 
836 combination of colony PCR across the chromosomal insertion site and correctly

837 localized expression of the fluorophore by microscopy. Deletion strains for TOM70,

838 TOM71, TOM20, GEM1, VPS27 and/or DID4 were generated by one step PCR-

839 mediated gene replacement with the indicated selection cassette using standard

840 techniques and oligo pairs listed in Table S1. Plasmid templates for gene replacement

841 were from the pRS series of vectors (Sikorski and Hieter, 1989). Correct insertion of the

842 selection cassette into the target gene was confirmed by colony PCR across the

843 chromosomal insertion site. Yeast strains constitutively expressing TOM70, TOM20,

844 TIM50 or the indicated mitochondrial carrier protein from the GPD promoter were

845 generated by integration of the expression cassette into yeast chr I (199456-199457).

846 Plasmids for integration of the GPD-driven expression cassette are described below.

847 Correct insertion of the expression cassette into chr I was confirmed by colony PCR

848 across the chromosomal insertion site.

Wild-type yeast strain AHY4706, which was rendered prototrophic with pHLUM

850 (see below) to prevent complications caused by amino acid auxotrophies in the BY

851 strain background, was used to quantify amino acid dependencies of MDC formation

852 and for analysis of whole cell metabolite levels. Wild-type yeast strains AHY5082,

853 AHY7053, AHY7620, AHY8529 and AHY8531 were used for super resolution and time-

854 lapse imaging. Wild-type and gem14 yeast strains AHY4706, AHY4057, AHY7808,

855 AHY7816, AHY7804, AHY7806 were used for quantification of MDC-dependent

856 removal of proteins from mitochondria and protein enrichment in the MDC. A complete

857 list of all strains used in this manuscript can be found in the Key Resources Table. 


\section{Plasmids}

860 Plasmids used in this study are listed in the Key Resources Table. pHLUM, a yeast

861 plasmid expressing multiple auxotrophic marker genes from their endogenous

862 promoters, was obtained from Addgene (\#40276) (Mulleder et al., 2012). pHyg-AID*-

863 6FLAG (Morawska and Ulrich, 2013) and pNH605-pGPD1-osTIR1 (Chan et al., 2018)

864 were described previously. To integrate GPD1-osTIR1 into the LEU2 locus, pNH605-

865 pGPD1-osTIR1 was digested with Swa1. pHyg-6FLAG was generated by inserting

866 6FLAG amplified from pHyg-AID*-6FLAG into Kpn1/Xba1 digested pHyg-AID*-6FLAG.

867 Plasmids for GPD-driven expression of TOM70, TOM20, ILV2, TIM50 or the indicated

868 mitochondrial carrier protein were generated by gateway mediated transfer of the

869 corresponding ORF (Harvard Institute of Proteomics) from pDONR201/221 into

870 pAG306GPD-ccdB chr 1 (Hughes and Gottschling, 2012) using Gateway ${ }^{\mathrm{TM}}$ LR

871 Clonase ${ }^{\mathrm{TM}}$ II Enzyme mix (ThermoFisher) according to the manufacturer's instructions.

872 To integrate the resulting expression plasmid into yeast chr I (199456-199457),

873 pAG306GPD-ORF chr 1 was digested with Notl. All insert sequences were verified by

874 the University of Utah Sequencing Core.

875

\section{$876 \quad$ Yeast Cell Culture and Media}

877 Yeast cells were grown exponentially for 15 hours at $30^{\circ} \mathrm{C}$ to a maximum density of

$8786 \times 10^{6}$ cells $/ \mathrm{mL}$ before the start of all experiments described in the paper, including MDC 879 and spot assays. This period of overnight log-phase growth was carried out to ensure

880 vacuolar and mitochondrial uniformity across the cell population and is essential for

881 consistent MDC activation. Cells were cultured as indicated in the Main Text and Figure 
882 Legends in media containing high amino acids (1\% yeast extract, $2 \%$ peptone, $0.005 \%$

883 adenine, $2 \%$ glucose) or low amino acids ( $0.67 \%$ yeast nitrogen base without amino

884 acids, $2 \%$ glucose, supplemented nutrients $0.074 \mathrm{~g} / \mathrm{L}$ each adenine, alanine, arginine,

885 asparagine, aspartic acid, cysteine, glutamic acid, glutamine, glycine, histidine, myo-

886 inositol, isoleucine, lysine, methionine, phenylalanine, proline, serine, threonine,

887 tryptophan, tyrosine, uracil, valine, $0.369 \mathrm{~g} / \mathrm{L}$ leucine, $0.007 \mathrm{~g} / \mathrm{L}$ para-aminobenzoic

888 acid). For growth in medium containing no amino acids ( $0.67 \%$ yeast nitrogen base

889 without amino acids, $2 \%$ glucose), cells were cultured in low amino acid medium and

890 then shifted to medium containing no amino acids at time of drug treatment. Where

891 casamino acids (CasAA) were added to low or no amino acid media, CasAA were

892 added at time of drug treatment to a final concentration of $2 \% \mathrm{w} / \mathrm{v}$. For single amino acid

893 re-addition experiments, individual amino acids were added to medium containing low

894 amino acids at the time of drug treatment. All amino acids were added to a final

895 concentration of $20 \mathrm{mg} / \mathrm{mL}$, except indicated otherwise, with the exception of cysteine

896 and tyrosine, which were added at final concentrations of $5 \mathrm{mg} / \mathrm{mL}$ and $1 \mathrm{mg} / \mathrm{mL}$

897 respectively, due to toxicity and/or solubility issues. Leucine and methionine catabolites

898 were added at a final concentration of $10 \mu \mathrm{M}$ at the time of drug treatment. Drugs were

899 added to cultures at final concentrations of Concanamycin A (500nM), Cycloheximide

$900(10 \mu \mathrm{g} / \mathrm{mL})$, Rapamycin (250 nM), Torin1 (5 $\mu \mathrm{M})$, Antimycin A (40 $\mu \mathrm{M})$, FCCP $(10 \mu \mathrm{M})$,

901 Oligomycin $(10 \mu \mathrm{M}), \mathrm{H}_{2} \mathrm{O}_{2}(10 \mu \mathrm{M}), \mathrm{CoCl}_{2}(1 \mathrm{mM})$, Tunicamycin $(5 \mu \mathrm{g} / \mathrm{ml})$ and BPS

$902(250 \mu \mathrm{M})$. Iron was added to cultures as $\left(\mathrm{NH}_{4}\right)_{2} \mathrm{Fe}\left(\mathrm{SO}_{4}\right)_{2}\left(\mathrm{H}_{2} \mathrm{O}\right)_{6}$ at a final concentration

903 of $2 \mathrm{mM}$. 


\section{Yeast MDC Assays}

906 For yeast MDC assays, overnight log-phase cell cultures were grown in the presence of

907 dimethyl sulfoxide (DMSO) or the indicated drug or metabolite for two hours. To acutely

908 inhibit V-ATPase function by Auxin-inducible degradation of Vma2, overnight log-phase

909 cell cultures were treated with $1 \mathrm{mM}$ Auxin in high amino acid medium for the indicated

910 times. For amino acid re-addition MDC assays, overnight log-phase cell cultures grown

911 in low amino acid medium were shifted to low amino acid medium supplemented with

912 the indicated amino acid and drug and grown for two hours. After incubation, cells were

913 harvested by centrifugation, resuspended in imaging buffer $(5 \% \mathrm{w} / \mathrm{v}$ Glucose, $10 \mathrm{mM}$

914 HEPES pH 7.6) and optical Z-sections of live yeast cells were acquired with an

915 Axiolmager M2 (Carl Zeiss) or, for super-resolution images, an Airyscan LSM800 (Carl

916 Zeiss) or Airyscan LSM880 (Carl Zeiss). The percentage of cells with MDCs was

917 quantified in each experiment at the two-hour time point from maximum intensity

918 projected wide field images generated in ZEN (Carl Zeiss). MDCs were identified as

919 Tom70-enriched, Tim50-negative structures of varying size and shape. In tom70 $\Delta$ and

920 tom70 $\Delta$ tom71 $\Delta$ strains, Tcd2, another MDC substrate (Hughes et al., 2016) was used

921 to identify MDCs. A single focal plane is displayed for all yeast images with the

922 exception of time-lapse images (see below).

923

924 Yeast Time-Lapse Imaging

925 For time-lapse imaging of yeast treated with concanamycin A, overnight log-phase

926 cultures grown in high amino acid medium were treated with concanamycin A for 30

927 minutes. Cells were harvested by centrifugation, resuspended in low amino acid 
928 medium plus Cas AA and concanamycin A, and pipetted into flow chamber slides as

929 previously described (Fees et al., 2017). Briefly, flow chambers were made using

930 standard microscope slides and coverslips. Strips of parafilm were used to seal a

931 coverslip to a slide and created the walls of the chamber. Flow chambers were coated

932 with concanavalin A prior to loading cells. Melted Vaseline was used to seal the

933 chamber. MDC formation was imaged every minute for 120 minutes. For time-lapse

934 imaging of yeast treated with cycloheximide, overnight log-phase cultures grown in high

935 amino acid medium were treated with cycloheximide for two minutes. Cells were

936 harvested by centrifugation, resuspended in low amino acid medium plus Cas AA and

937 cycloheximide, and pipetted into flow chamber slides. MDC formation was imaged every

938 minute for 120 minutes. For time-lapse imaging of yeast treated with rapamycin,

939 overnight log-phase cultures grown in high amino acid medium were treated with

940 rapamycin for 15 minutes. Cells were harvested by centrifugation, resuspended in low

941 amino acid medium plus Cas AA and rapamycin, and pipetted into flow chamber slides.

942 MDC formation was imaged every minute for 119 minutes. $300 \mathrm{~nm}$ optical Z-sections of

943 live yeast cells were acquired with an Airyscan LSM880 in Airyscan fast mode. Time-

944 lapse images and movies of yeast MDC formation show maximum intensity projections.

$946 \quad$ Fluorescent Staining

947 In order to visualize mitochondrial DNA in yeast, cells were incubated with $2 \mu \mathrm{g} / \mathrm{mL}$

948 DAPI in high amino acid medium for 30 minutes at RT. To visualize mitochondrial

949 membrane potential in yeast, cells were re-isolated by centrifugation, washed with

950 imaging buffer and stained with $50 \mathrm{nM}$ tetramethylrhodamine methyl ester (TMRM) for 
95115 minutes at RT. In case of concanamycin A treatment, media were supplemented with

$9522 \mathrm{mM}$ iron during the treatment period to prevent loss of the mitochondrial membrane

953 potential as previously described (Hughes et al., 2020). To visualize vacuolar acidity in

954 yeast, cells were re-isolated by centrifugation and stained with $200 \mu \mathrm{M}$ Quinacrine in

955 high amino acid medium supplemented with 50 mM HEPES pH 7.6 for 10 minutes at

$95630^{\circ} \mathrm{C}$ followed by a 5-minute incubation on ice. Prior to imaging, cells were washed

957 twice with imaging buffer.

958

959 Microscopy and image analysis

960 For quantification of MDC formation or fluorescence intensities, $200 \mathrm{~nm}$ optical Z-

961 sections of live yeast cells were acquired with an Axiolmager M2 (Carl Zeiss) equipped

962 with an edge 4.2 CMOS camera (PCO) and $63 \times$ or $100 \times$ oil-immersion objectives (Carl

963 Zeiss, Plan Apochromat, NA 1.4). Super resolution images showing MDC formation in

964 live yeast cells were acquired with an LSM800 or LSM880 (Carl Zeiss) equipped with an

965 Airyscan detector (Carl Zeiss) and 63× oil-immersion objective (Carl Zeiss, Plan

966 Apochromat, NA 1.4). Widefield images were acquired with ZEN (Carl Zeiss),

967 processed with Fiji (Schindelin et al., 2012), and represent single Z-sections. Super-

968 resolution images were acquired with ZEN (Carl Zeiss), processed using the automated

969 Airyscan processing algorithm in ZEN (Carl Zeiss) and Fiji, and represent single 200 nM

970 Z-sections (with the exception of time-lapse images). Individual channels of all images

971 were minimally adjusted in Fiji to match the fluorescence intensities between channels

972 for better visualization. Line-scan analysis and measurements of MDC size were

973 performed on non-adjusted, single Z-sections from super resolution images. To quantify 
974 the average MDC size, the diameter of spherical MDCs that had a visible lumen was

975 measured using the line tool in Fiji. Tubular MDCs or smaller Tom70-enriched

976 structures lacking Tim50 were excluded from the analysis since they likely represent

977 broken down or growing MDCs. Fluorescence intensity analysis was performed on non-

978 adjusted, maximum intensity projected wide-field images. To quantify the residual

979 mitochondrial fluorescence upon MDC formation, the mean fluorescence intensity was

980 measured along the entire mitochondrial tubule, excluding the MDC, using the freehand

981 selection tool in Fiji and normalized to the untreated control sample. For analysis of

982 fluorescence enrichment in the MDC, mean fluorescence intensities were measured in

983 along the brightest part of the MDC using the freehand line tool in Fiji and normalized to

984 the mean fluorescence intensity in the directly adjacent mitochondrial tubule.

986 Yeast Growth Assays

987 To analyze growth of yeast cells on plates containing high levels of single amino acids,

988 five-fold serial dilutions of over-night log-phase cultures grown in low amino acid

989 medium were prepared in low amino acid medium and $3 \mu$ of each dilution were spotted

990 onto the agar medium (3\% w/v agar) denoted in each Figure Legend. Total cells plated

991 in each dilution spot were $5,000,1,000,200,40$, and 8.

992

\section{Protein Preparation and Immunoblotting}

994 For western blot analysis of total protein levels, overnight log-phase cultures were

995 treated as indicated and $5 \times 10^{7}$ yeast cells were re-isolated by centrifugation, washed

996 with $\mathrm{ddH}_{2} \mathrm{O}$ and incubated in $0.1 \mathrm{M} \mathrm{NaOH}$ for 5 min at RT. Subsequently, cells were re- 
997 isolated by centrifugation at $16,000 \times \mathrm{g}$ for 10 minutes at $4^{\circ} \mathrm{C}$ and lysed for 5 minutes at $99895^{\circ} \mathrm{C}$ in SDS-Lysis buffer (10mM Tris pH 6.8, $100 \mathrm{mM} \mathrm{NaCl,} 1 \mathrm{mM}$ EDTA, 1mM EGTA, $9991 \%(w / v)$ SDS). Upon lysis, protein concentrations were determined by bicinchoninic 1000 acid assay (G Biosciences) and samples were denatured in Laemmli buffer (63 mM Tris $1001 \mathrm{pH}$ 6.8, 2\% (w/v) SDS, 10\% (v/v) glycerol, $1 \mathrm{mg} / \mathrm{ml}$ bromophenol blue, $1 \%$ (v/v) $\beta$ -

1002 mercaptoethanol) for 5 minutes at $95^{\circ} \mathrm{C}$. To separate proteins based on molecular 1003 weight, equal amounts of protein were subjected to SDS polyacrylamide gel

1004 electrophoresis and transferred to PVDF membrane (Millipore) by wet transfer.

1005 Nonspecific antibody binding was blocked by incubation with TBS containing 5\% (w/v)

1006 dry milk (Sigma Aldrich) for one hour at RT. After incubation with the primary antibodies 1007 (listed in Key Resources Table) for two hours at RT or at $4^{\circ} \mathrm{C}$ overnight, membranes 1008 were washed five times with TBS and incubated with secondary antibody (goat-anti1009 rabbit/mouse HRP-conjugated,1:2000 in TBS + 5\% dry milk, Sigma Aldrich) for 45

1010 minutes at RT. Membranes were washed five times with TBS, enhanced

1011 chemiluminescence solution (Thermo Fisher) was applied and the antibody signal was 1012 detected with a BioRad Chemidoc MP system. All blots were exported as TIFFs using 1013 ImageLab 6.0 (BioRad) and cropped in Adobe Photoshop CC. Western blots show one 1014 representative blot from $n=3$ replicates performed in parallel with the associated MDC 1015 assay.

1016

\section{Extraction of Whole Cell Metabolites from Yeast}

1018 For analysis of whole cell metabolite levels, cells were grown exponentially in the 1019 indicated media for 15 hours to a maximum density of $6 \times 10^{6}$ cells $/ \mathrm{mL}$ and treated for 
1020 two hours with the indicated drugs. $5 \times 10^{7}$ total yeast cells were harvested by

1021 centrifugation, washed once with water, and cell pellets were shock frozen in liquid

1022 nitrogen. Whole cell metabolites were extracted from yeast cell pellets as previously

1023 described with slight modifications (Canelas et al., 2009). Briefly, the internal standard

1024 succinic- $d_{4}$ acid (Sigma Aldrich 10907HD) at a final concentration of $1 \mu \mathrm{g} / \mathrm{sample.}$

1025 Subsequently, $5 \mathrm{~mL}$ of boiling $75 \%$ EtOH were added to each cell pellet, followed by

1026 vortex mixing and incubation at $90^{\circ} \mathrm{C}$ for five minutes. Cell debris were removed by

1027 centrifugation and the supernatant was transferred to new tubes and dried en vacuo.

1028 Pooled quality control samples were made by removing a fraction of collected

1029 supernatant from each sample and process blanks were made using only extraction

1030 solvent and no cell culture pellet.

1031

\section{GC-MS Analysis}

1033 Amino acid composition analysis was performed on an Agilent 7200 GC-QToF mass

1034 spectrometer fitted with an Agilent 7890 gas chromatograph and an Agilent 7693A

1035 automatic liquid sampler. Dried samples were suspended in $40 \mu \mathrm{L}$ of $40 \mathrm{mg} / \mathrm{mL} \mathrm{O}-$

1036 methoxylamine hydrochloride (MP Biomedicals) in dry pyridine and incubated for one

1037 hour at $30^{\circ} \mathrm{C} .25 \mu \mathrm{L}$ of this solution was added to auto sampler vials. $60 \mu \mathrm{L}$ of N-methyl-

1038 N-trimethylsilyltrifluoracetamide (Pierce) was added automatically via the auto sampler

1039 and incubated for 30 minutes at $37^{\circ} \mathrm{C}$ with shaking. After incubation, $1 \mu \mathrm{L}$ of the

1040 prepared sample was injected into the gas chromatograph inlet in the split mode with

1041 the inlet temperature held at $250^{\circ} \mathrm{C}$. A $25: 1$ split ratio was used for analysis. The gas

1042 chromatograph had an initial temperature of $60^{\circ} \mathrm{C}$ for one minute followed by a 
$104310^{\circ} \mathrm{C} / \mathrm{min}$ ramp to $325^{\circ} \mathrm{C}$ and a hold time of 2 minutes. A 30-meter Agilent Zorbax DB-

1044 5MS with 10m Duraguard capillary column was employed for chromatographic

1045 separation. Helium was used as the carrier gas at a rate of $1 \mathrm{~mL} / \mathrm{min}$.

1046 Data was collected using MassHunter software (Agilent). Metabolites were identified

1047 and their peak area was recorded using MassHunter Quant. Metabolite identity was

1048 established using a combination of an in-house metabolite library developed using pure

1049 purchased standards, the NIST library and the Fiehn Library. Values for each metabolite

1050 were normalized to the internal standard in each sample and are displayed as fold

1051 change compared to the control sample. All error bars show the mean \pm standard error

1052 from three biological replicates analyzed in the same GC-MS run.

1053

1054 Quantification and Statistical Analysis

1055 All experiments were repeated at least three times. All attempts at replication were 1056 successful. Sample sizes were as large as possible to be representative, but of a 1057 manageable size for quantifications. Specifically, for yeast MDC assays, $N=$ three 1058 replicates, with $n=100$ cells for each replicate, for quantification of MDC size, $N=30$ -

105950 MDCs from $N=3-5$ experiments and for fluorescence intensity analysis $N=45$

1060 cells from $N=$ three replicates with $N=15$ cells per replicate. No data were excluded

1061 from the analyses. No randomization or blinding was used as all experiments were 1062 performed with defined laboratory reagents and yeast strains of known genotypes. 1063 1064 


\section{REFERENCES}

1067 Abe, Y., Shodai, T., Muto, T., Mihara, K., Torii, H., Nishikawa, S., Endo, T., and Kohda,

1068

D. (2000). Structural basis of presequence recognition by the mitochondrial protein

1069 import receptor Tom20. Cell 100, 551-560.

1070 Aliu, E., Kanungo, S., and Arnold, G.L. (2018). Amino acid disorders. Ann Transl Med 6, 1071471.

1072 Barbet, N.C., Schneider, U., Helliwell, S.B., Stansfield, I., Tuite, M.F., and Hall, M.N.

1073 (1996). TOR controls translation initiation and early G1 progression in yeast. Molecular 1074 biology of the cell 7, 25-42.

1075 Beugnet, A., Tee, A.R., Taylor, P.M., and Proud, C.G. (2003). Regulation of targets of 1076 mTOR (mammalian target of rapamycin) signalling by intracellular amino acid 1077 availability. The Biochemical journal 372, 555-566.

1078 Brachmann, C.B., Davies, A., Cost, G.J., Caputo, E., Li, J., Hieter, P., and Boeke, J.D. 1079 (1998). Designer deletion strains derived from Saccharomyces cerevisiae S288C: a 1080 useful set of strains and plasmids for PCR-mediated gene disruption and other 1081 applications. Yeast 14, 115-132.

1082 Canelas, A.B., ten Pierick, A., Ras, C., Seifar, R.M., van Dam, J.C., van Gulik, W.M., 1083 and Heijnen, J.J. (2009). Quantitative evaluation of intracellular metabolite extraction 1084 techniques for yeast metabolomics. Anal Chem 81, 7379-7389.

1085 Cardenas, M.E., Cutler, N.S., Lorenz, M.C., Di Como, C.J., and Heitman, J. (1999). The 1086 TOR signaling cascade regulates gene expression in response to nutrients. Genes \& 1087 development 13, 3271-3279. 
1088 Chan, L.Y., Mugler, C.F., Heinrich, S., Vallotton, P., and Weis, K. (2018). Non-invasive 1089 measurement of mRNA decay reveals translation initiation as the major determinant of 1090 mRNA stability. eLife 7, pii: e32536. doi: 10.7554/eLife.32536.

1091 Chen, E.J., and Kaiser, C.A. (2003). LST8 negatively regulates amino acid biosynthesis 1092 as a component of the TOR pathway. The Journal of cell biology $161,333-347$.

1093 Chen, K.L., Ven, T.N., Crane, M.M., Brunner, M.L.C., Pun, A.K., Helget, K.L., Brower, 1094 K., Chen, D.E., Doan, H., Dillard-Telm, J.D., et al. (2020). Loss of vacuolar acidity 1095 results in iron-sulfur cluster defects and divergent homeostatic responses during aging 1096 in Saccharomyces cerevisiae. Geroscience. doi: 10.1007/s11357-020-00159-3.

1097 Dimmer, K.S., Fritz, S., Fuchs, F., Messerschmitt, M., Weinbach, N., Neupert, W., and 1098 Westermann, B. (2002). Genetic basis of mitochondrial function and morphology in 1099 Saccharomyces cerevisiae. Molecular biology of the cell 13, 847-853.

1100 Donaton, M.C., Holsbeeks, I., Lagatie, O., Van Zeebroeck, G., Crauwels, M., 1101 Winderickx, J., and Thevelein, J.M. (2003). The Gap1 general amino acid permease 1102 acts as an amino acid sensor for activation of protein kinase A targets in the yeast 1103 Saccharomyces cerevisiae. Molecular microbiology 50, 911-929.

1104 English, A.M., Kornmann, B., Shaw J.M., and Hughes, A.L. (2020). ER-mitochondria 1105 contacts promote mitochondrial-derived compartment biogenesis. Submitted.

1106 Efeyan, A., Comb, W.C., and Sabatini, D.M. (2015). Nutrient-sensing mechanisms and 1107 pathways. Nature 517, 302-310. 
1108 Fees, C.P., Estrem, C., and Moore, J.K. (2017). High-resolution Imaging and Analysis of

1109 Individual Astral Microtubule Dynamics in Budding Yeast. J Vis Exp 122, doi:

$1110 \quad 10.3791 / 55610$.

1111 Frederick, R.L., McCaffery, J.M., Cunningham, K.W., Okamoto, K., and Shaw, J.M.

1112 (2004). Yeast Miro GTPase, Gem1p, regulates mitochondrial morphology via a novel

1113 pathway. The Journal of cell biology 167, 87-98.

1114 Grauslund, M., Didion, T., Kielland-Brandt, M.C., and Andersen, H.A. (1995). BAP2, a

1115 gene encoding a permease for branched-chain amino acids in Saccharomyces

1116 cerevisiae. Biochim Biophys Acta 1269, 275-280.

1117 Hara, K., Yonezawa, K., Weng, Q.P., Kozlowski, M.T., Belham, C., and Avruch, J.

1118 (1998). Amino acid sufficiency and mTOR regulate p70 S6 kinase and elF-4E BP1

1119 through a common effector mechanism. J Biol Chem 273, 14484-14494.

1120 Hatakeyama, R., and De Virgilio, C. (2019). TORC1 specifically inhibits microautophagy

1121 through ESCRT-0. Curr Genet 65, 1243-1249.

1122 Hazelwood, L.A., Daran, J.M., van Maris, A.J., Pronk, J.T., and Dickinson, J.R. (2008).

1123 The Ehrlich pathway for fusel alcohol production: a century of research on

1124 Saccharomyces cerevisiae metabolism. Appl Environ Microbiol 74, 2259-2266.

1125 Hines, V., Brandt, A., Griffiths, G., Horstmann, H., Brutsch, H., and Schatz, G. (1990).

1126 Protein import into yeast mitochondria is accelerated by the outer membrane protein

1127 MAS70. EMBO J 9, 3191-3200.

1128 Hinnebusch, A.G. (2005). Translational regulation of GCN4 and the general amino acid 1129 control of yeast. Annu Rev Microbiol 59, 407-450. 
1130 Hughes, A.L., and Gottschling, D.E. (2012). An early age increase in vacuolar pH limits 1131 mitochondrial function and lifespan in yeast. Nature 492, 261-265.

1132 Hughes, A.L., Hughes, C.E., Henderson, K.A., Yazvenko, N., and Gottschling, D.E.

1133 (2016). Selective sorting and destruction of mitochondrial membrane proteins in aged

1134 yeast. eLife 5, pii: e13943.

1135 Hughes, C.E., Coody, T.K., Jeong, M.Y., Berg, J.A., Winge, D.R., and Hughes, A.L. 1136 (2020). Cysteine Toxicity Drives Age-Related Mitochondrial Decline by Altering Iron

1137 Homeostasis. Cell 180, 296-310.

1138 Hurley, J.H., and Emr, S.D. (2006). The ESCRT complexes: structure and mechanism 1139 of a membrane-trafficking network. Annual review of biophysics and biomolecular 1140 structure 35, 277-298.

1141 Katzmann, D.J., Odorizzi, G., and Emr, S.D. (2002). Receptor downregulation and

1142 multivesicular-body sorting. Nat Rev Mol Cell Biol 3, 893-905.

1143 Klionsky, D.J., Herman, P.K., and Emr, S.D. (1990). The fungal vacuole: composition, 1144 function, and biogenesis. Microbiological reviews 54, 266-292.

1145 Knebel, B., Strassburger, K., Szendroedi, J., Kotzka, J., Scheer, M., Nowotny, B., 1146 Mussig, K., Lehr, S., Pacini, G., Finner, H., et al. (2016). Specific Metabolic Profiles and

1147 Their Relationship to Insulin Resistance in Recent-Onset Type 1 and Type 2 Diabetes. J 1148 Clin Endocrinol Metab 101, 2130-2140.

1149 Lin, C.H., MacGurn, J.A., Chu, T., Stefan, C.J., and Emr, S.D. (2008). Arrestin-related 1150 ubiquitin-ligase adaptors regulate endocytosis and protein turnover at the cell surface. 1151 Cell 135, 714-725. 
1152 Ljungdahl, P.O. (2009). Amino-acid-induced signalling via the SPS-sensing pathway in 1153 yeast. Biochemical Society transactions 37, 242-247.

1154 Ljungdahl, P.O., and Daignan-Fornier, B. (2012). Regulation of amino acid, nucleotide, 1155 and phosphate metabolism in Saccharomyces cerevisiae. Genetics 190, 885-929.

1156 Lynch, C.J., and Adams, S.H. (2014). Branched-chain amino acids in metabolic 1157 signalling and insulin resistance. Nat Rev Endocrinol 10, 723-736.

1158 Merz, S., and Westermann, B. (2009). Genome-wide deletion mutant analysis reveals 1159 genes required for respiratory growth, mitochondrial genome maintenance and 1160 mitochondrial protein synthesis in Saccharomyces cerevisiae. Genome Biol 10, R95.

1161 Moczko, M., Ehmann, B., Gartner, F., Honlinger, A., Schafer, E., and Pfanner, N.

1162 (1994). Deletion of the receptor MOM19 strongly impairs import of cleavable preproteins 1163 into Saccharomyces cerevisiae mitochondria. J Biol Chem 269, 9045-9051.

1164 Moczko, M., Gartner, F., and Pfanner, N. (1993). The protein import receptor MOM19 of 1165 yeast mitochondria. FEBS Lett 326, 251-254.

1166 Morawska, M., and Ulrich, H.D. (2013). An expanded tool kit for the auxin-inducible 1167 degron system in budding yeast. Yeast 30, 341-351.

1168 Mulleder, M., Capuano, F., Pir, P., Christen, S., Sauer, U., Oliver, S.G., and Ralser, M.

1169 (2012). A prototrophic deletion mutant collection for yeast metabolomics and systems 1170 biology. Nature biotechnology 30, 1176-1178.

1171 Newgard, C.B., An, J., Bain, J.R., Muehlbauer, M.J., Stevens, R.D., Lien, L.F., Haqq, 1172 A.M., Shah, S.H., Arlotto, M., Slentz, C.A., et al. (2009). A branched-chain amino acid- 
1173 related metabolic signature that differentiates obese and lean humans and contributes

1174 to insulin resistance. Cell Metab 9, 311-326.

1175 Nikko, E., and Pelham, H.R. (2009). Arrestin-mediated endocytosis of yeast plasma

1176 membrane transporters. Traffic 10, 1856-1867.

1177 Noda, T., and Ohsumi, Y. (1998). Tor, a phosphatidylinositol kinase homologue,

1178 controls autophagy in yeast. J Biol Chem 273, 3963-3966.

1179 Ohya, Y., Umemoto, N., Tanida, I., Ohta, A., lida, H., and Anraku, Y. (1991). Calcium-

1180 sensitive cls mutants of Saccharomyces cerevisiae showing a Pet- phenotype are

1181 ascribable to defects of vacuolar membrane $\mathrm{H}(+)$-ATPase activity. J Biol Chem 266,

1182 13971-13977.

1183 Palmieri, F., and Monne, M. (2016). Discoveries, metabolic roles and diseases of

1184 mitochondrial carriers: A review. Biochim Biophys Acta 1863, 2362-2378.

1185 Rabinowitz, J.D., and White, E. (2010). Autophagy and metabolism. Science 330, 1344-

11861348.

1187 Risinger, A.L., Cain, N.E., Chen, E.J., and Kaiser, C.A. (2006). Activity-dependent

1188 reversible inactivation of the general amino acid permease. Molecular biology of the cell

$1189 \quad 17,4411-4419$.

1190 Rubio-Texeira, M., and Kaiser, C.A. (2006). Amino acids regulate retrieval of the yeast

1191 general amino acid permease from the vacuolar targeting pathway. Molecular biology of

1192 the cell $17,3031-3050$. 
1193 Ruiz, S.J., van 't Klooster, J.S., Bianchi, F., and Poolman, B. (2017). Growth inhibition 1194 by amino acids inSaccharomyces cerevisiae. BioRxiv,

1195 doi: https://doi.org/10.1101/222224.

1196 Ryan, M.T., Muller, H., and Pfanner, N. (1999). Functional staging of ADP/ATP carrier

1197 translocation across the outer mitochondrial membrane. J Biol Chem 274, 20619-

119820627.

1199 Saxton, R.A., and Sabatini, D.M. (2017). mTOR Signaling in Growth, Metabolism, and

1200 Disease. Cell 168, 960-976.

1201 Schindelin, J., Arganda-Carreras, I., Frise, E., Kaynig, V., Longair, M., Pietzsch, T.,

1202 Preibisch, S., Rueden, C., Saalfeld, S., Schmid, B., et al. (2012). Fiji: an open-source

1203 platform for biological-image analysis. Nat Methods 9, 676-682.

1204 Schlossmann, J., Lill, R., Neupert, W., and Court, D.A. (1996). Tom71, a novel

1205 homologue of the mitochondrial preprotein receptor Tom70. J Biol Chem 271, 17890-

120617895.

1207 Sheff, M.A., and Thorn, K.S. (2004). Optimized cassettes for fluorescent protein tagging 1208 in Saccharomyces cerevisiae. Yeast 21, 661-670.

1209 Sikorski, R.S., and Hieter, P. (1989). A system of shuttle vectors and yeast host strains

1210 designed for efficient manipulation of DNA in Saccharomyces cerevisiae. Genetics 122,

1211 19-27.

1212 Sollner, T., Pfaller, R., Griffiths, G., Pfanner, N., and Neupert, W. (1990). A

1213 mitochondrial import receptor for the ADP/ATP carrier. Cell 62, 107-115. 
1214 Soultoukis, G.A., and Partridge, L. (2016). Dietary Protein, Metabolism, and Aging.

1215 Annu Rev Biochem 85, 5-34.

1216 Steger, H.F., Sollner, T., Kiebler, M., Dietmeier, K.A., Pfaller, R., Trulzsch, K.S.,

1217 Tropschug, M., Neupert, W., and Pfanner, N. (1990). Import of ADP/ATP carrier into

1218 mitochondria: two receptors act in parallel. The Journal of cell biology 111, 2353-2363.

1219 Thoreen, C.C., Kang, S.A., Chang, J.W., Liu, Q., Zhang, J., Gao, Y., Reichling, L.J.,

1220 Sim, T., Sabatini, D.M., and Gray, N.S. (2009). An ATP-competitive mammalian target

1221 of rapamycin inhibitor reveals rapamycin-resistant functions of mTORC1. J Biol Chem

$1222 \quad 284,8023-8032$.

1223 Wang, T.J., Larson, M.G., Vasan, R.S., Cheng, S., Rhee, E.P., McCabe, E., Lewis,

1224 G.D., Fox, C.S., Jacques, P.F., Fernandez, C., et al. (2011). Metabolite profiles and the 1225 risk of developing diabetes. Nature medicine 17, 448-453.

1226 Weber, R.A., Yen, F.S., Nicholson, S.P.V., Alwaseem, H., Bayraktar, E.C., Alam, M.,

1227 Timson, R.C., La, K., Abu-Remaileh, M., Molina, H., et al. (2020). Maintaining Iron

1228 Homeostasis Is the Key Role of Lysosomal Acidity for Cell Proliferation. Mol Cell 77, 1229 645-655.

1230 Wellen, K.E., and Thompson, C.B. (2010). Cellular metabolic stress: considering how

1231 cells respond to nutrient excess. Mol Cell 40, 323-332.

1232 Wiemken, A., and Dürr, M. (1974). Characterization of amino acid pools in the vacuolar 1233 compartment of Saccharomyces cerevisiae. Archives of microbiology 101, 45-57. 
1234 Xu, F., Tavintharan, S., Sum, C.F., Woon, K., Lim, S.C., and Ong, C.N. (2013).

1235 Metabolic signature shift in type 2 diabetes mellitus revealed by mass spectrometry-

1236 based metabolomics. J Clin Endocrinol Metab 98, E1060-1065.

1237 Yamamoto, H., Esaki, M., Kanamori, T., Tamura, Y., Nishikawa, S., and Endo, T.

1238 (2002). Tim50 is a subunit of the TIM23 complex that links protein translocation across

1239 the outer and inner mitochondrial membranes. Cell 111, 519-528.

1240 Yambire, K.F., Rostosky, C., Watanabe, T., Pacheu-Grau, D., Torres-Odio, S.,

1241 Sanchez-Guerrero, A., Senderovich, O., Meyron-Holtz, E.G., Milosevic, I., Frahm, J., et

1242 al. (2019). Impaired lysosomal acidification triggers iron deficiency and inflammation in

1243 vivo. eLife 8, pii: e51031. doi: 10.7554/eLife.51031.

1244 Yin, Z., Pascual, C., and Klionsky, D.J. (2016). Autophagy: machinery and regulation.

1245 Microb Cell 3, 588-596. 
AoRxiv preprint doi: https://doi.org/10.1101/2020.03.13.991091; this version posted March 14, 2020. The copyright holder for this preprint

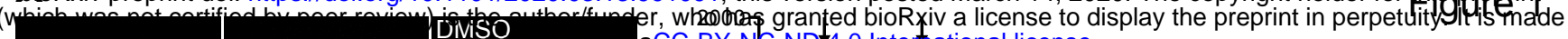
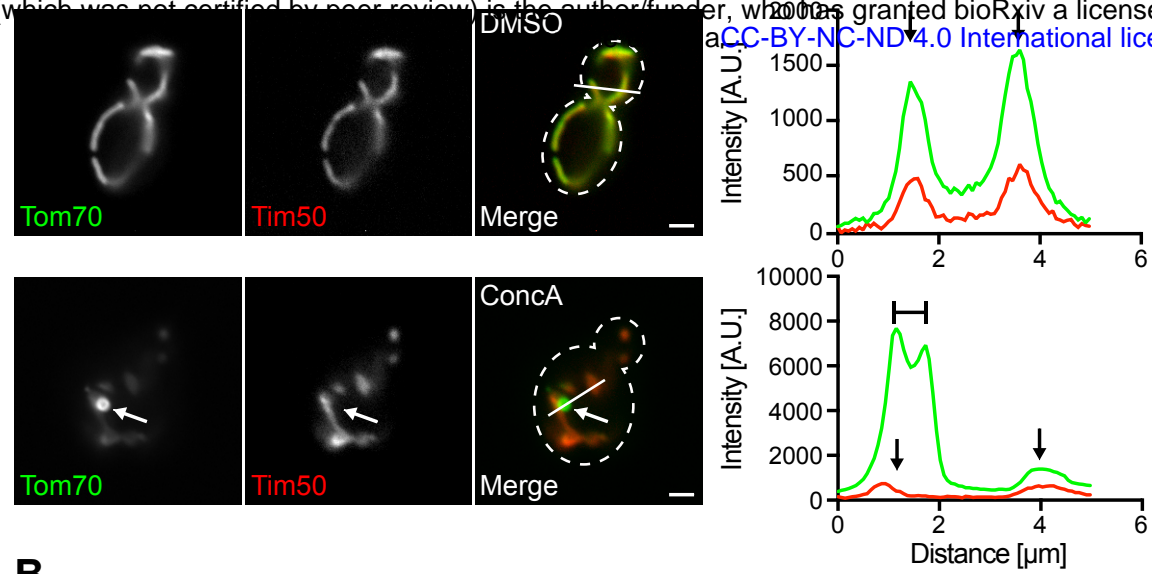

B
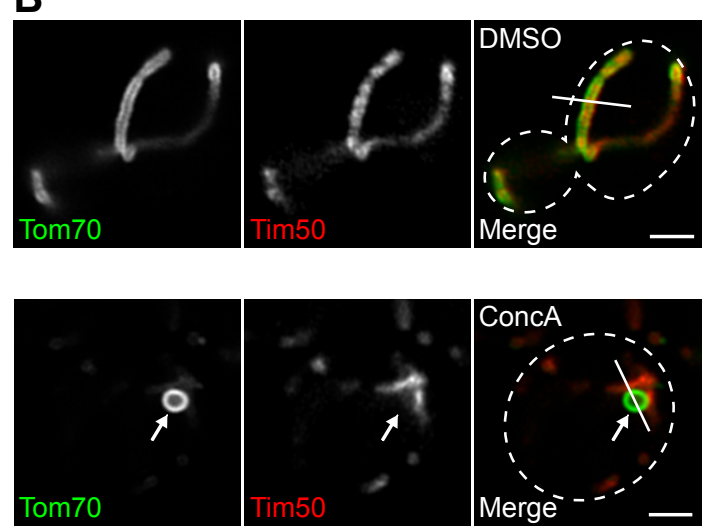

D

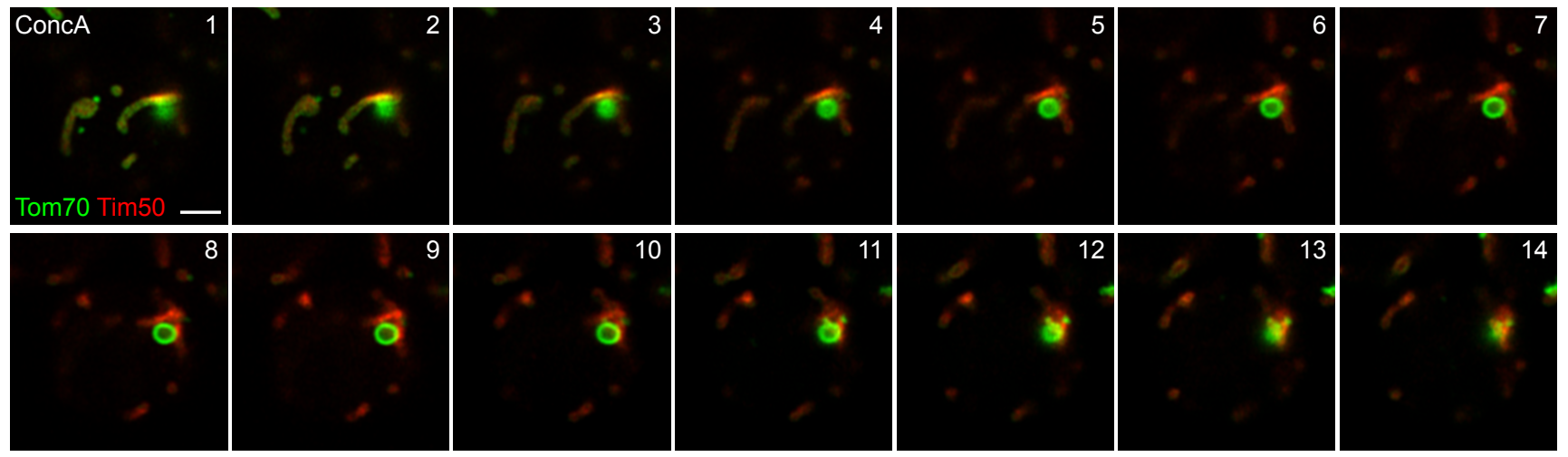

$0.15 \mu \mathrm{m}$ intervals

E

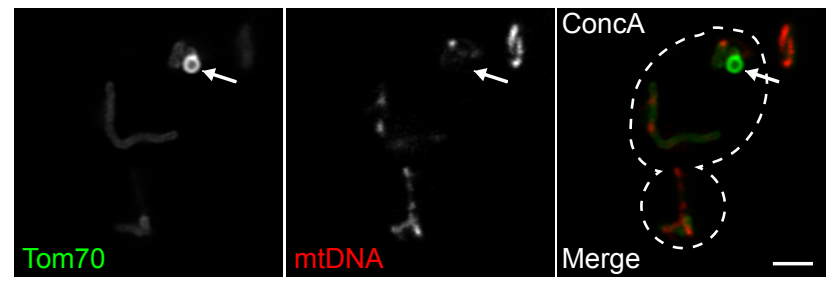

F

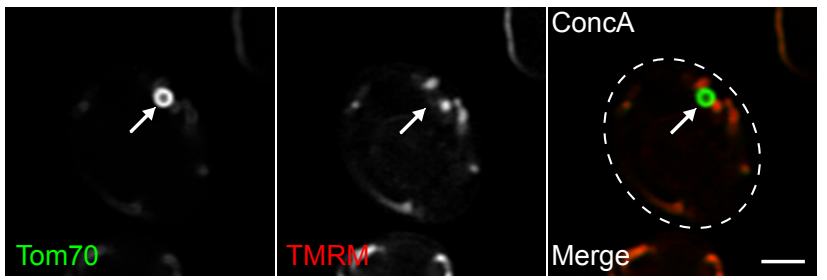

G

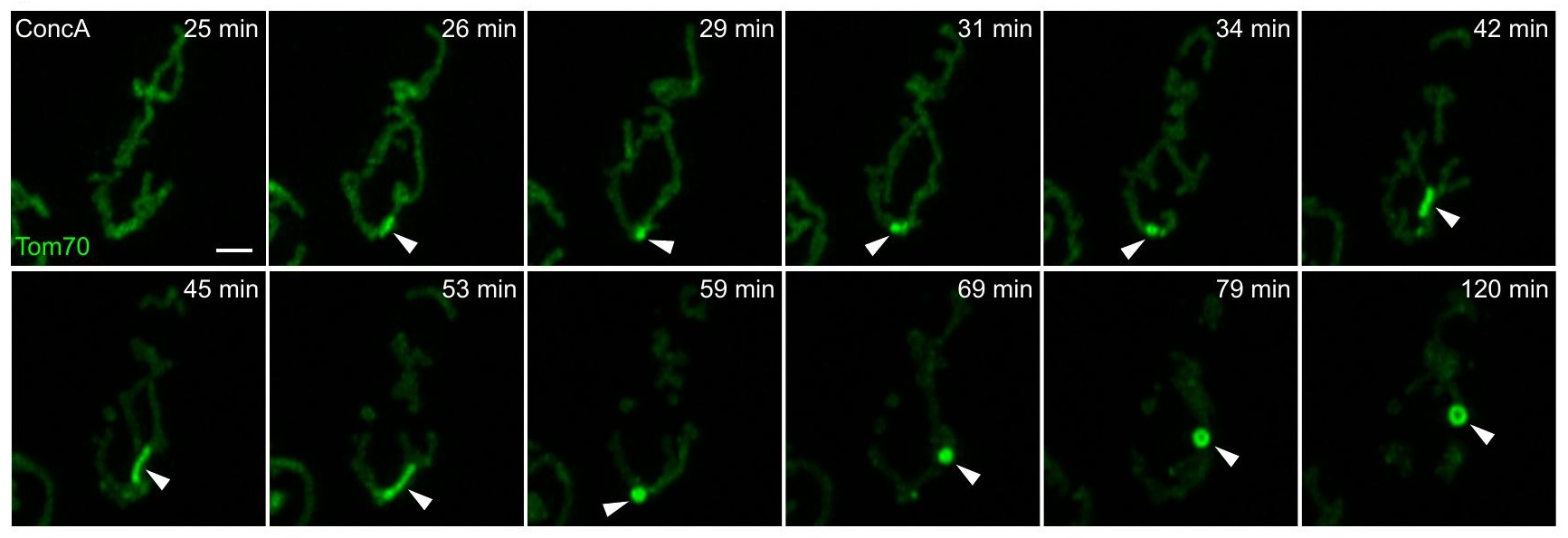


AoRxiv preprint doi: https://doi.org/10.1101/2020.03.13.991091; this ver\$Bn posted March 14, 2020. The copyright holder for this preprint (which was not certified by peer review) is the author/funder, who has granted bioRxiv a license to display the preprint in perpetuity. It is made
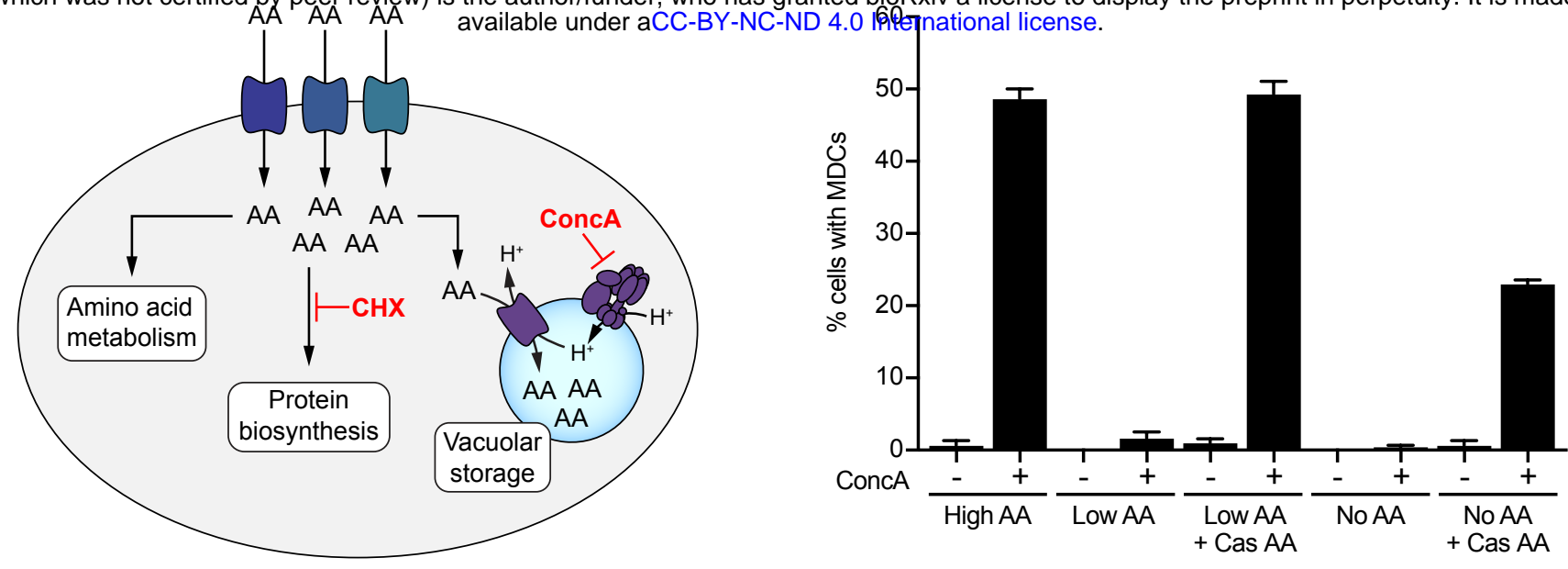

C
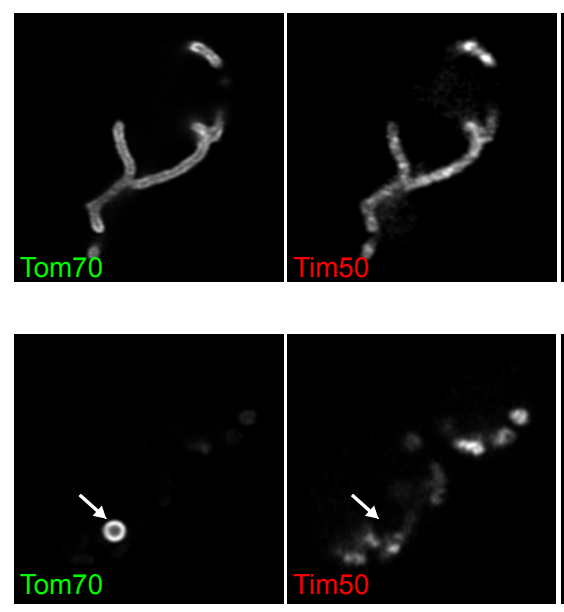

E

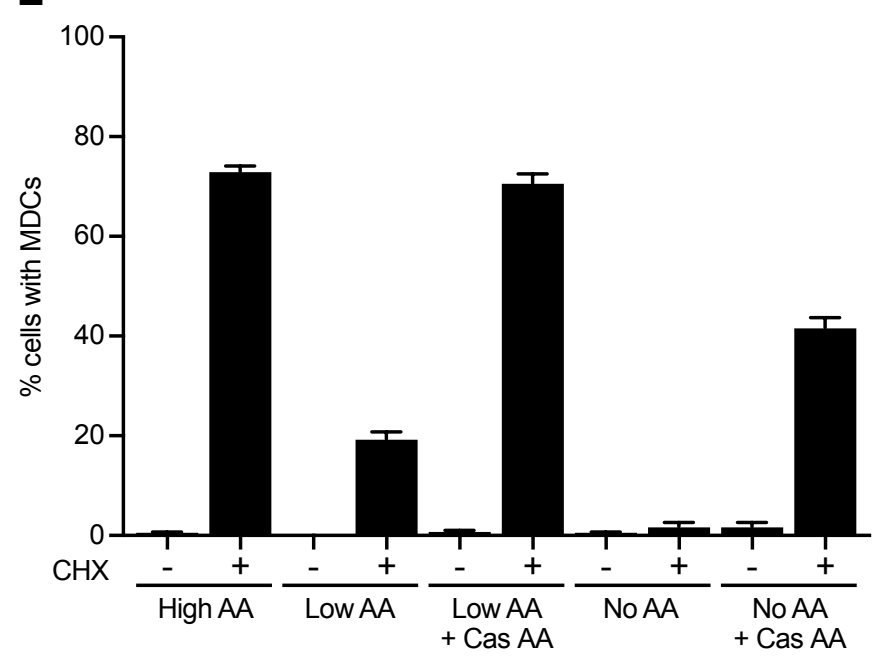

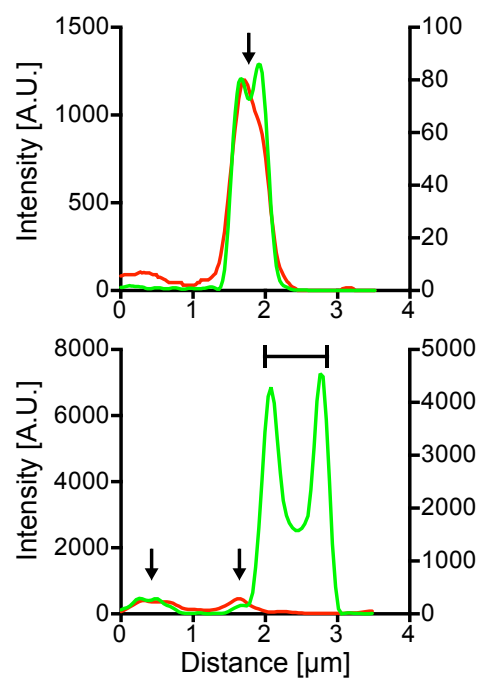

D

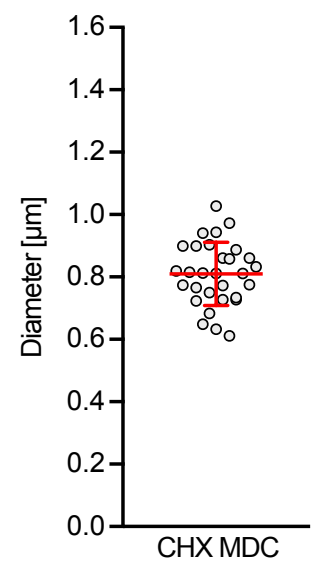


ApRxiv preprint doi: https://doi.org/10.1101/2020.03.13.991091; this version posted March 14, $\mathbf{B} 20$. The copyright holder for this preprint (which sfas not certified by peer review) is the author/funder, who has granted bioRxiv a license to display the prepript indigrpetuity. It is made
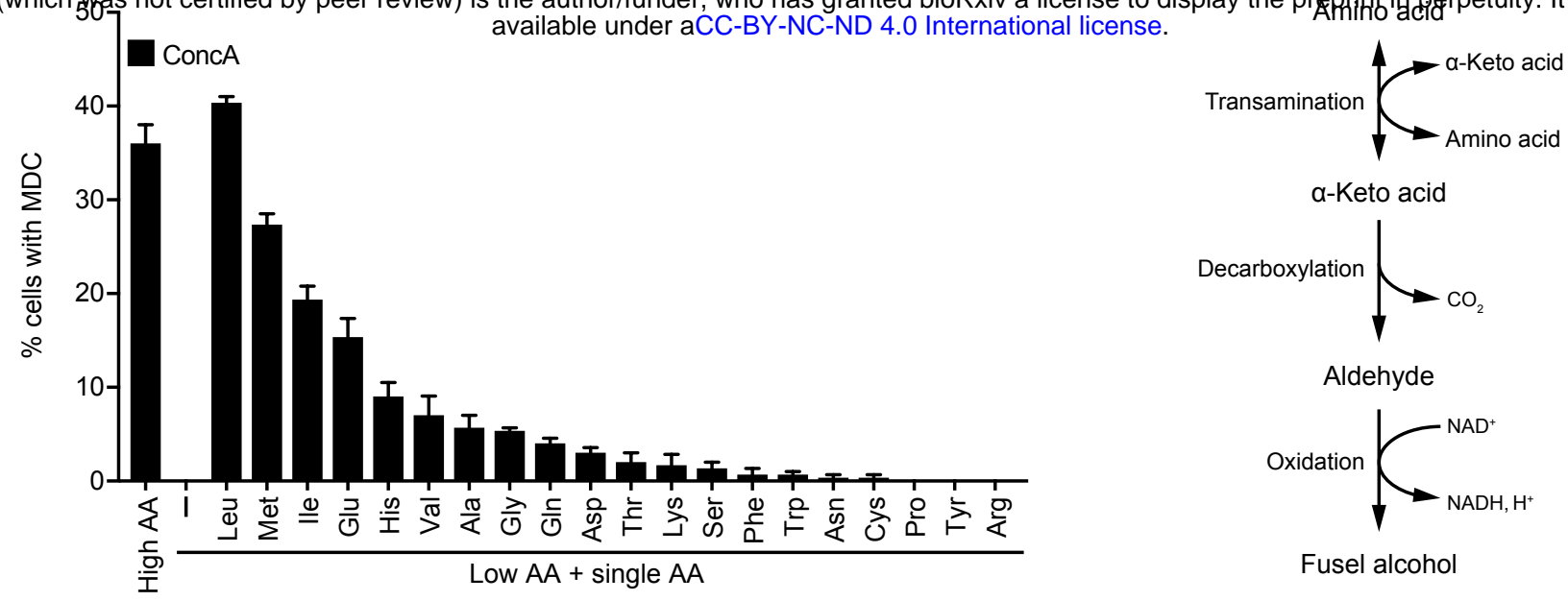

Aldehyde

C

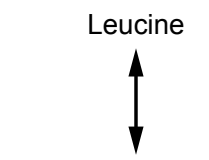

Ketoisocaproic acid $(\mathrm{KIC})$

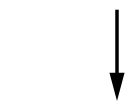

Isovaleraldehyde

(IVA)

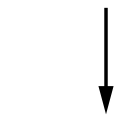

Isoamylalcohol (IAA)

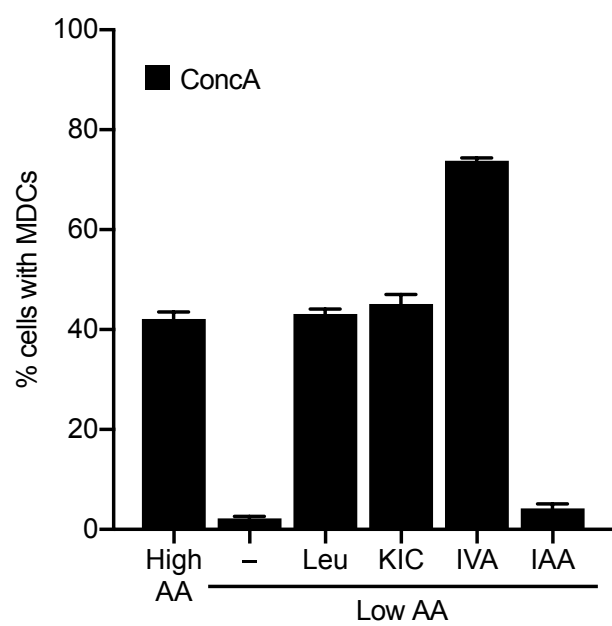

D

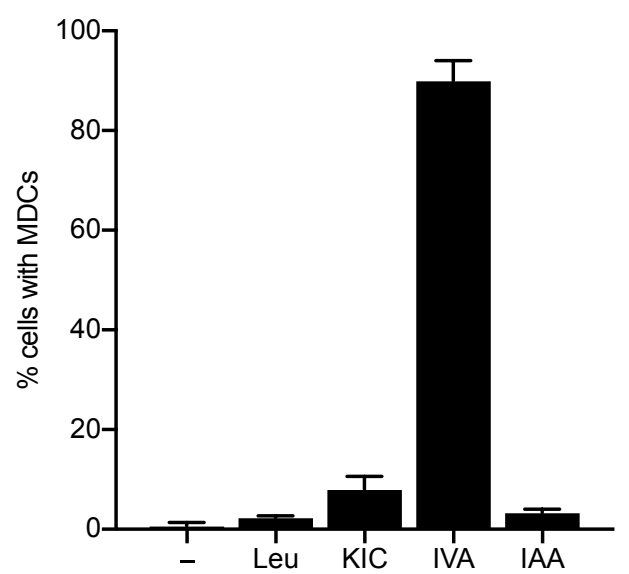

E
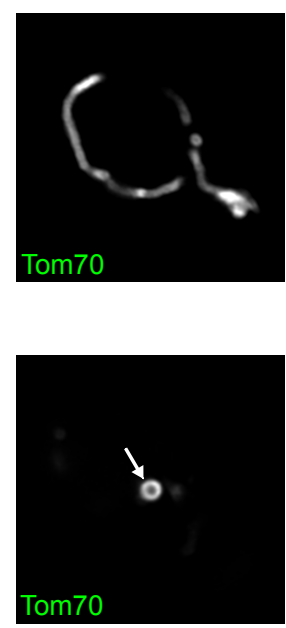
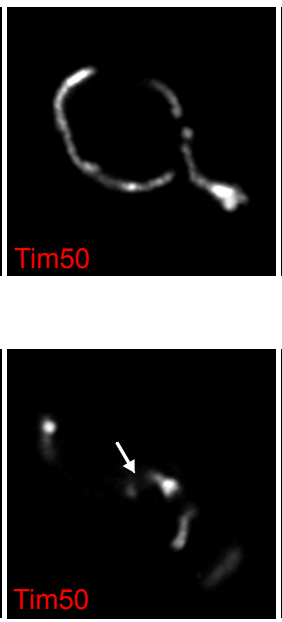
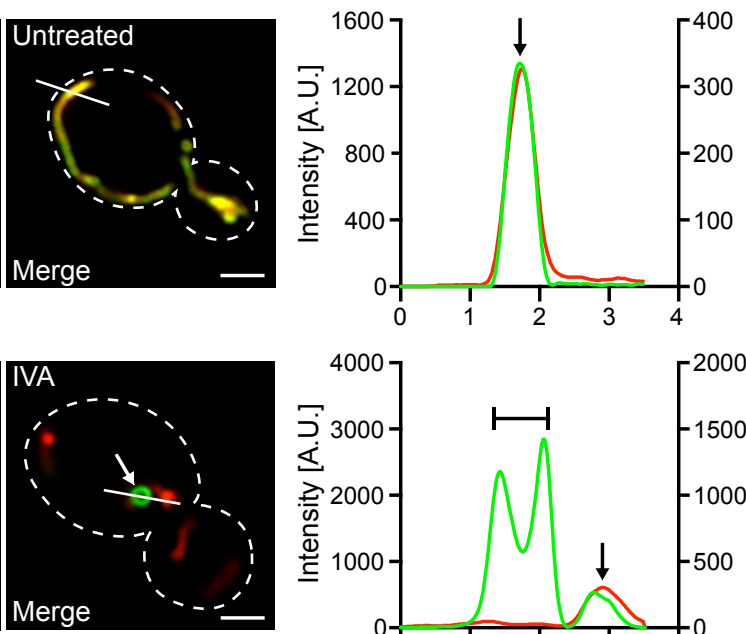

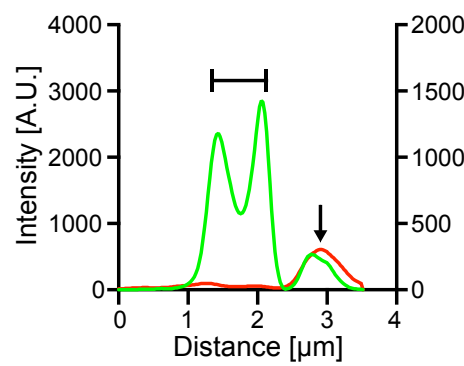

F

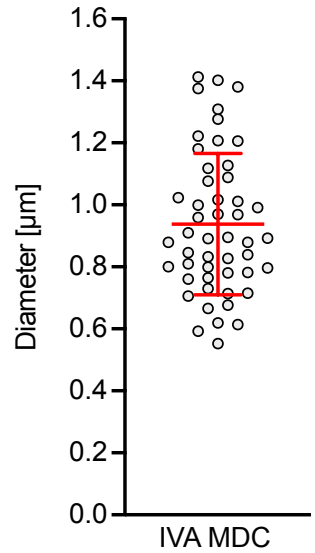


ApRxiv preprint doi: https://doi.org/10.1101/2020.03.13.991091; version posted March 14, 2020. The copyright holder for this preprint (which was not certified by peer review) is the author/funder, who has grantoed bioRxiv a license to display the preprint in perpetuity. It is made available under aCC-BY-NC-ND 4.d Internationa Trênse.
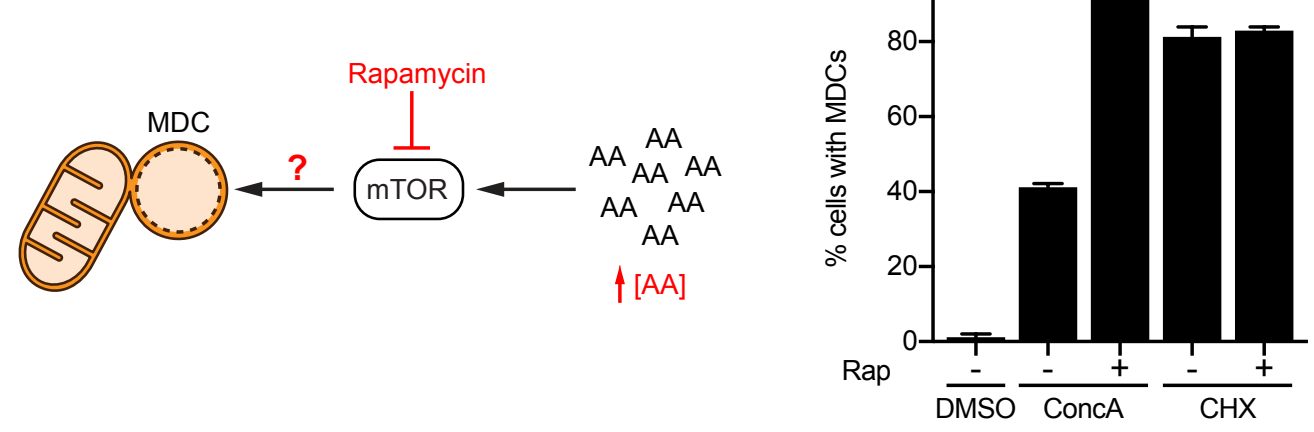

C
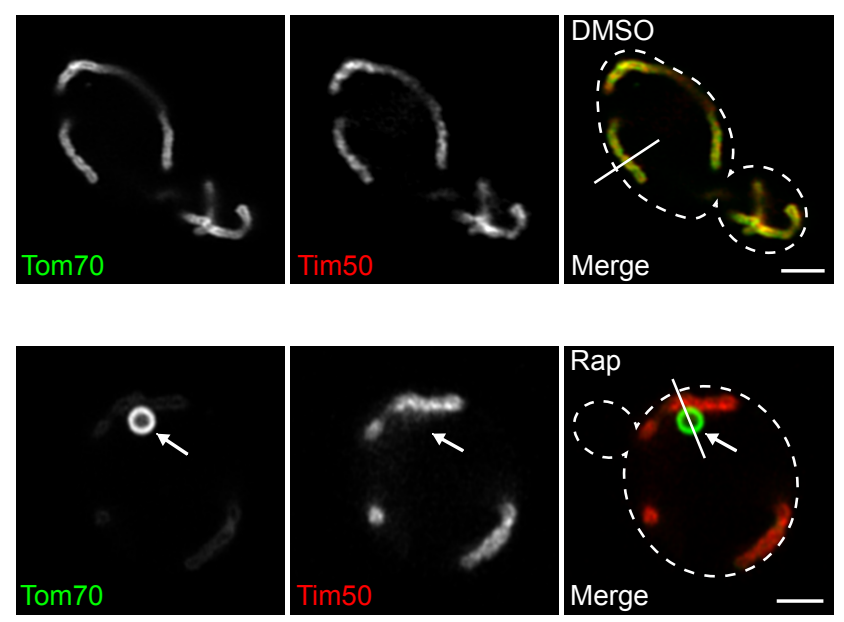

E

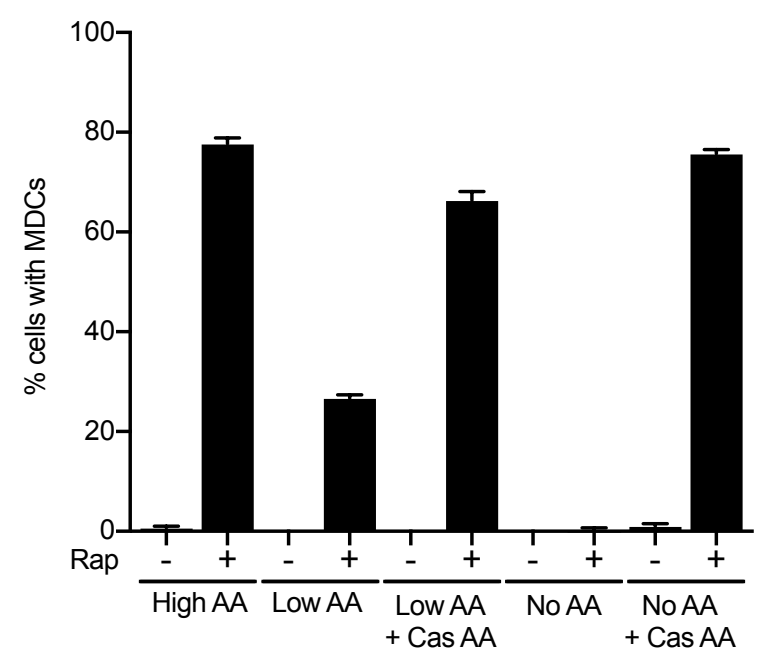

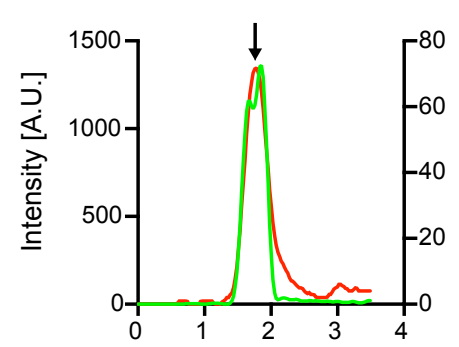

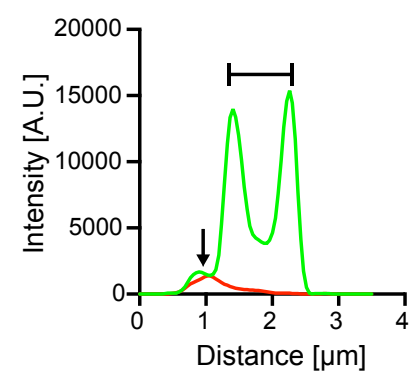

$\mathbf{F}$

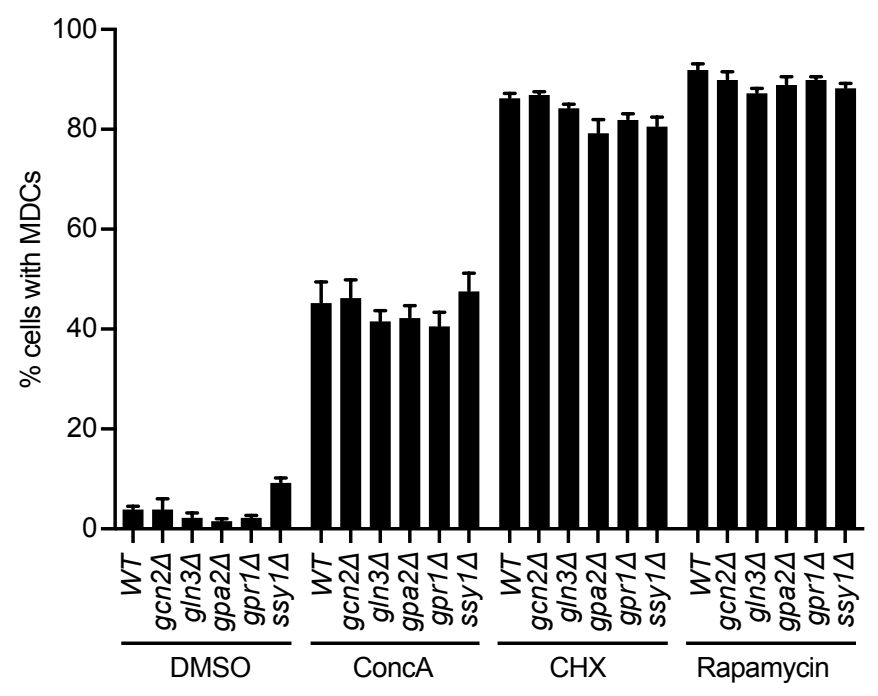


bARxiv preprint doi: https://doi.org/10.1101/2020.03. B.991091; this version posted March 14, 2020. The copyright holder for this preprint (which not_certified by peer review) is the author/funder goto has granted bioRxiv a license to display the preprint in perpetuity. It is made
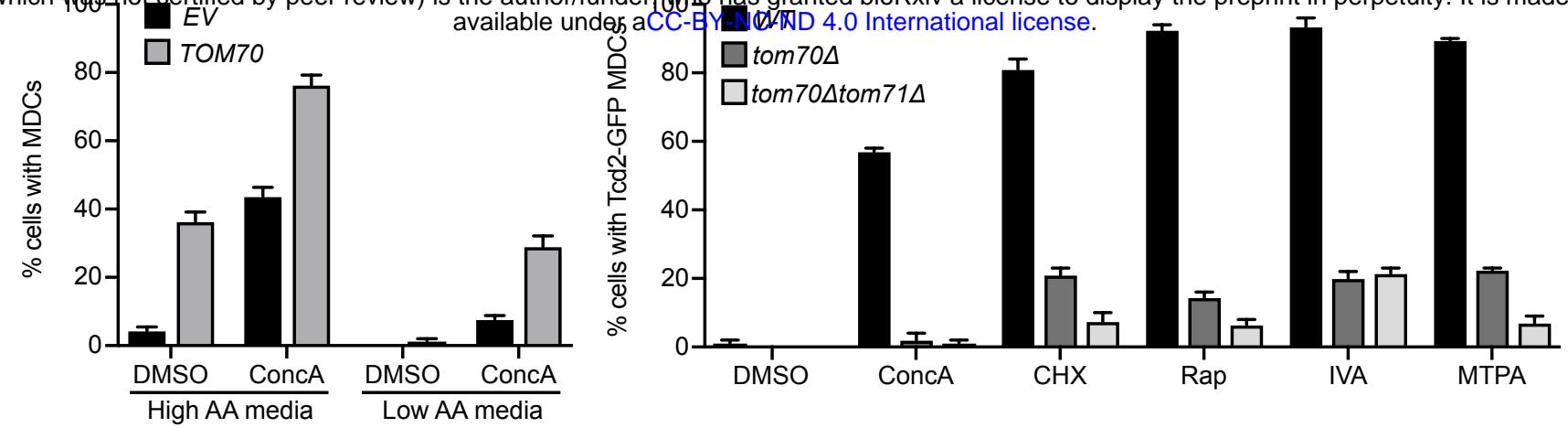

C

D
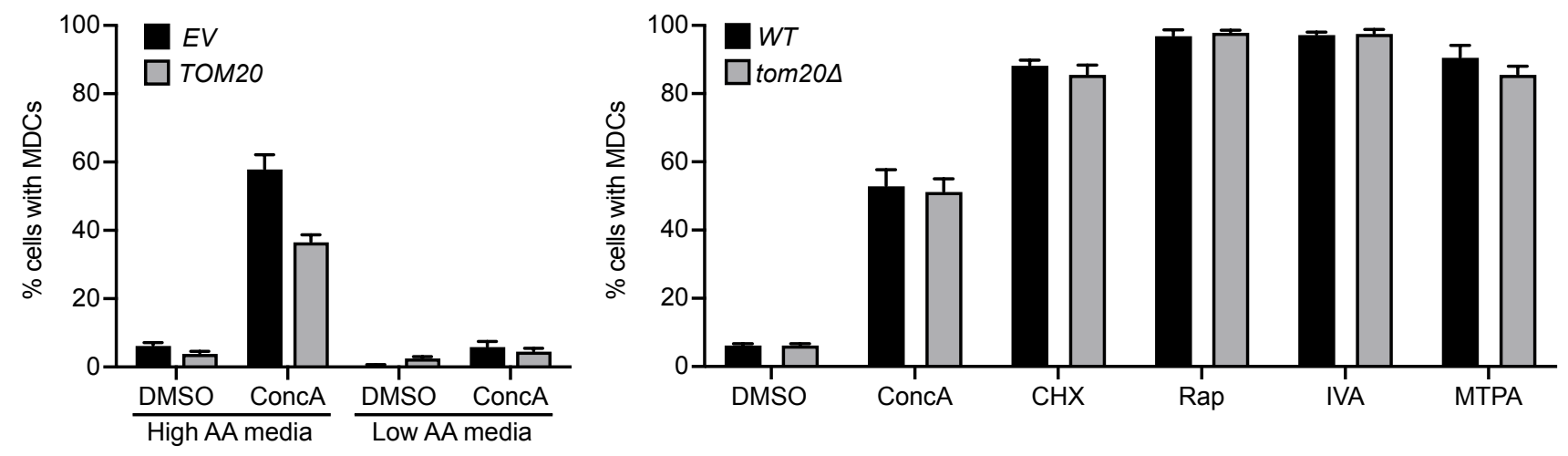

E

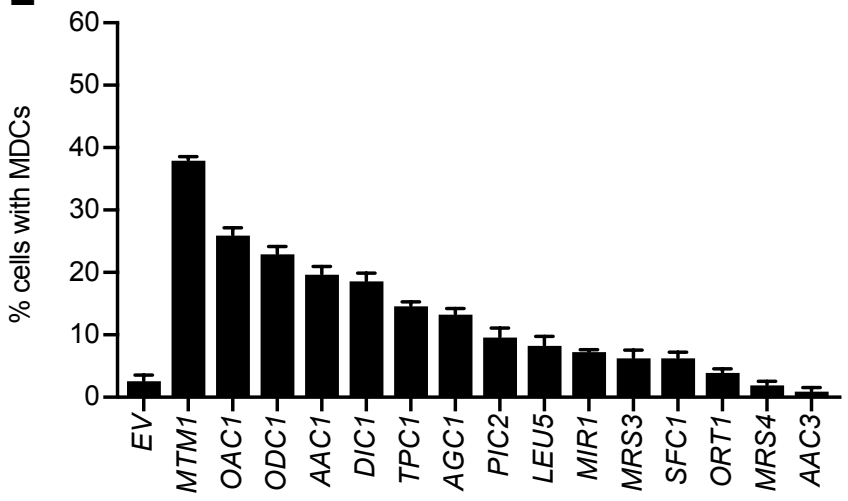

High AA media (untreated)

F

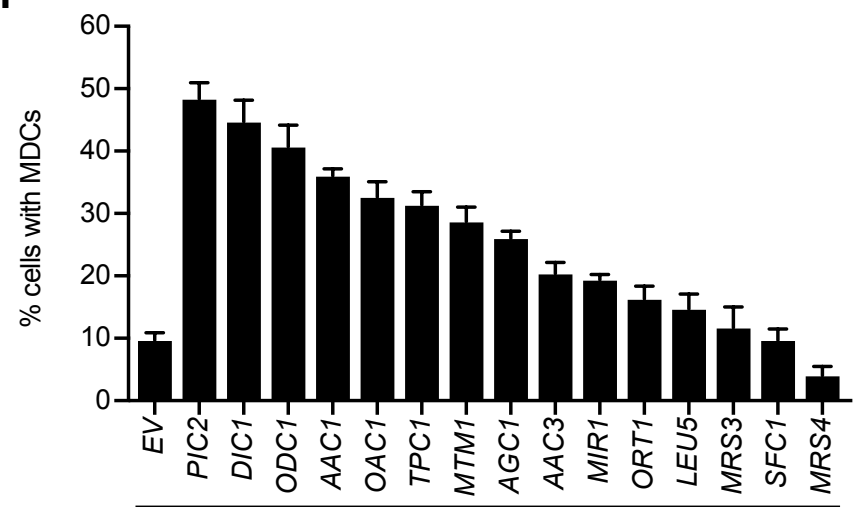

Low AA media (ConcA) 
AoRxiv preprint doi: https://doi.org/10.1101/2020.03.13.991091; this version postBd March 14, 2020. The copyright halder for this preprint

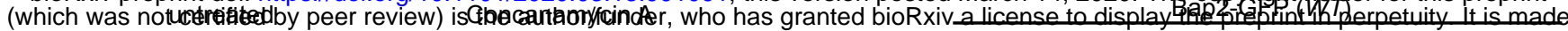

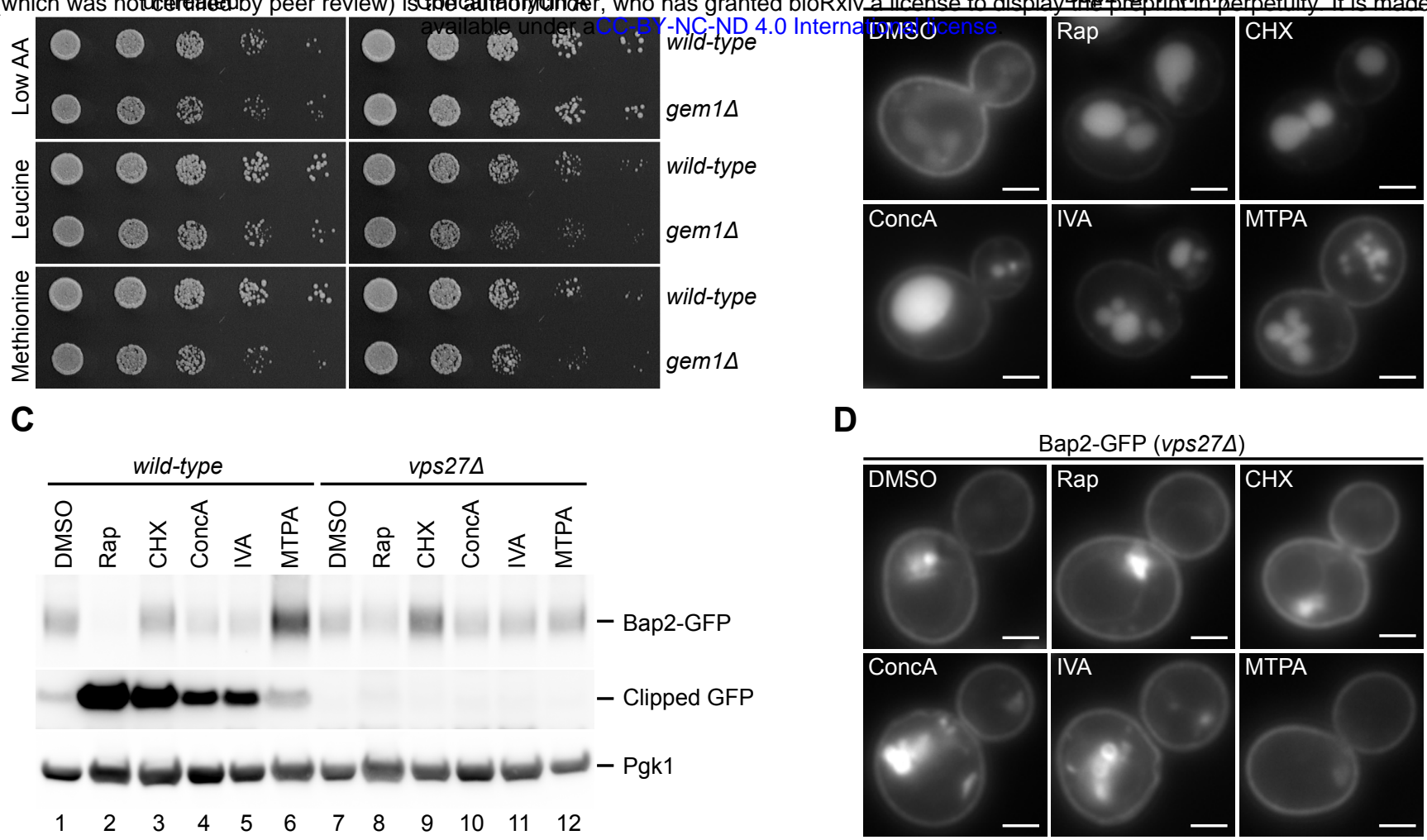

$\mathbf{E}$

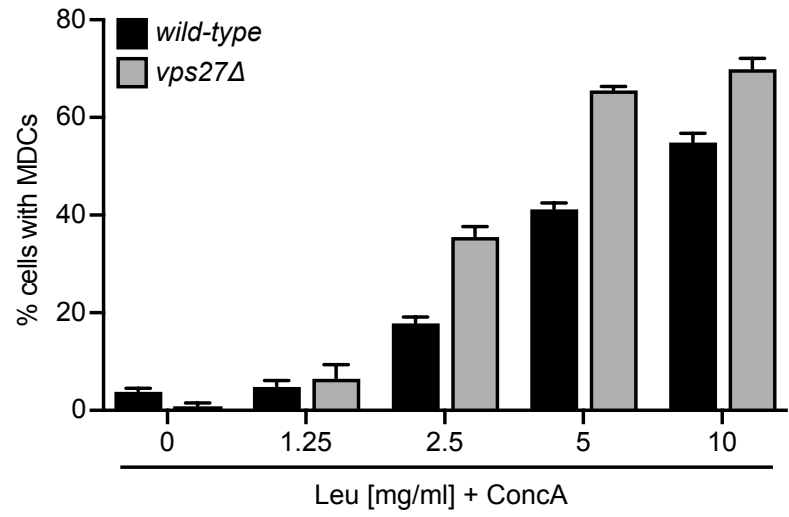

G

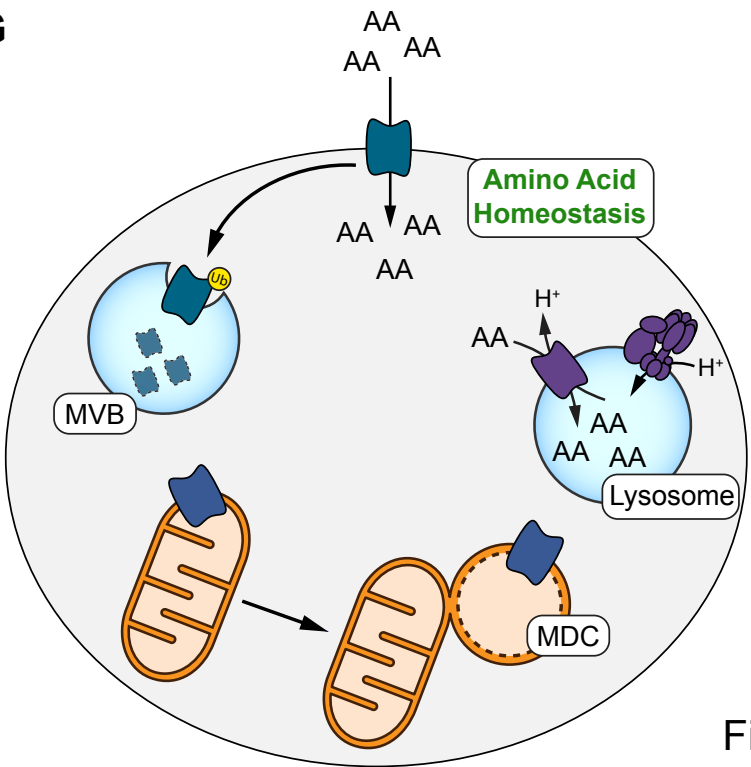

$\mathbf{F}$

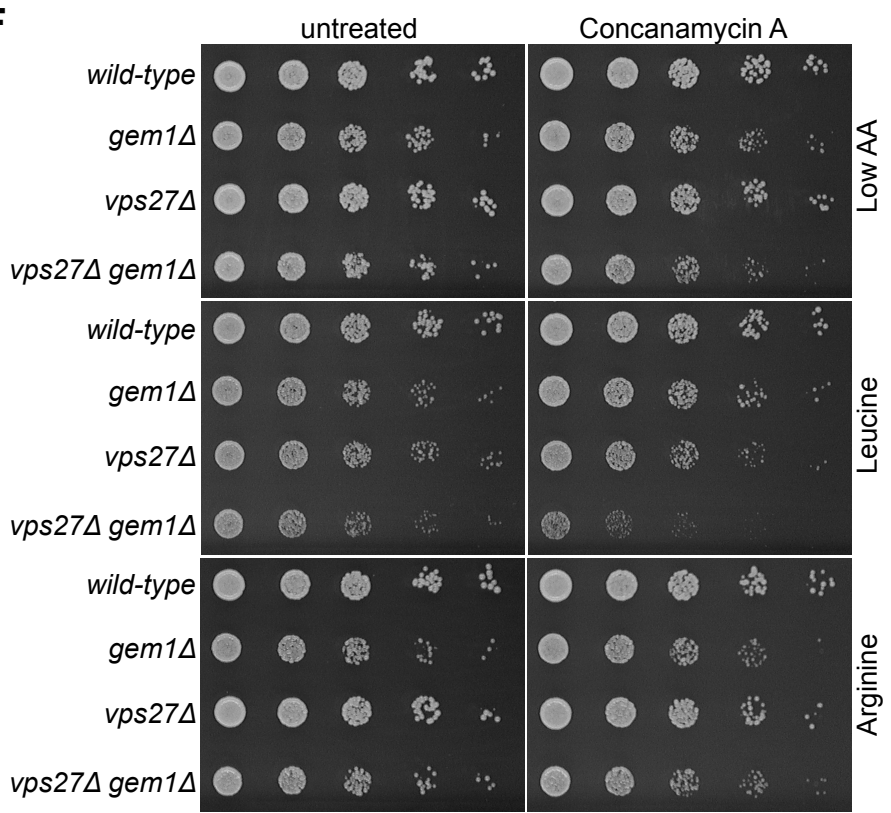

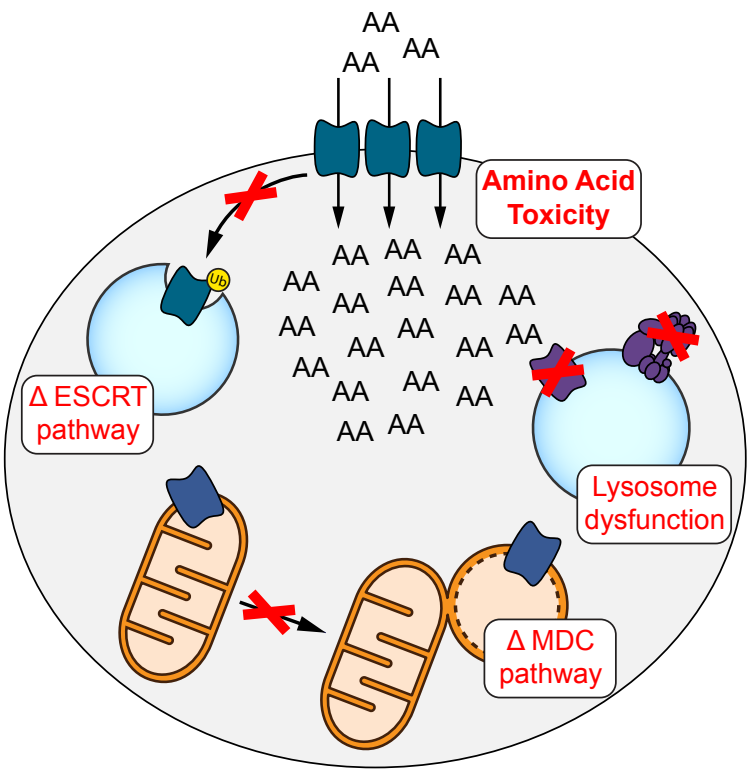


AoRxiv preprint doi: https://doi.org/10.1101/2020.03.13B91091; this version posted March 14, 2020. Che copyright holder for this preprint (which was not certified by peer review) is the author/funder, who has granted bioRxiv a license to display the preprint in perpetuity. It is made available under aCC-BY-NGS-NPARA-Itteknnational license.

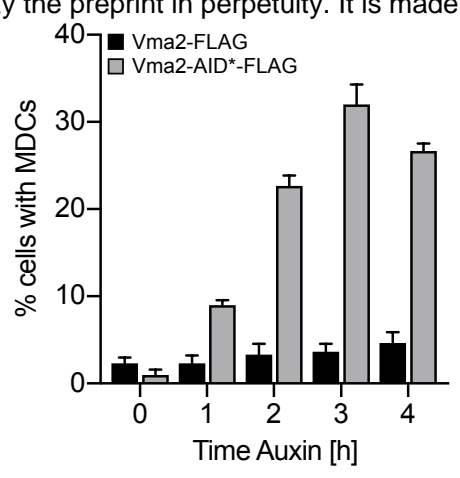

D

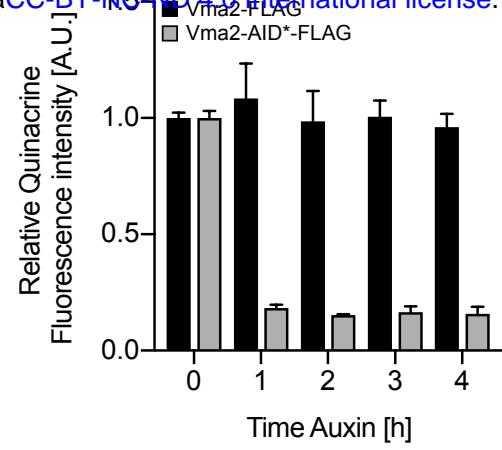

Vma2-FLAG

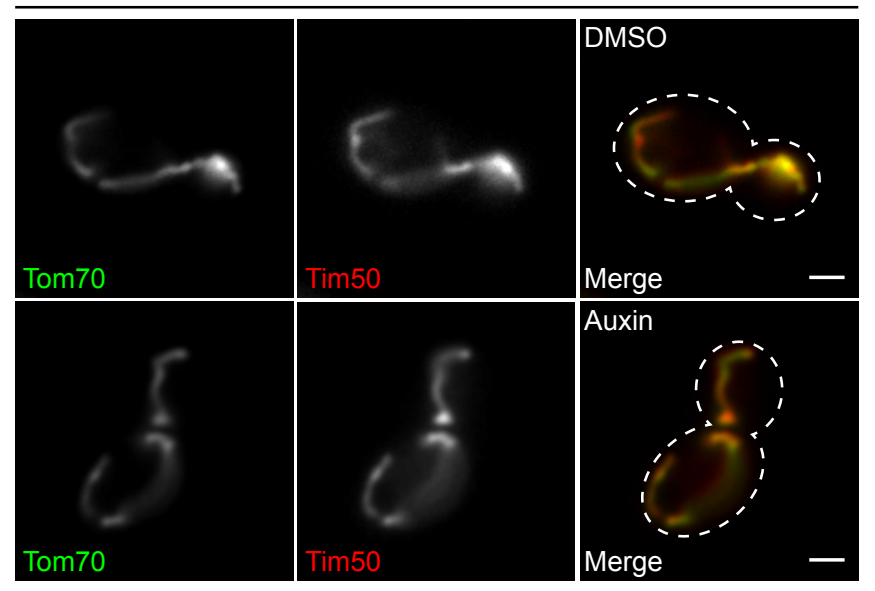

Vma2-AID*-FLAG

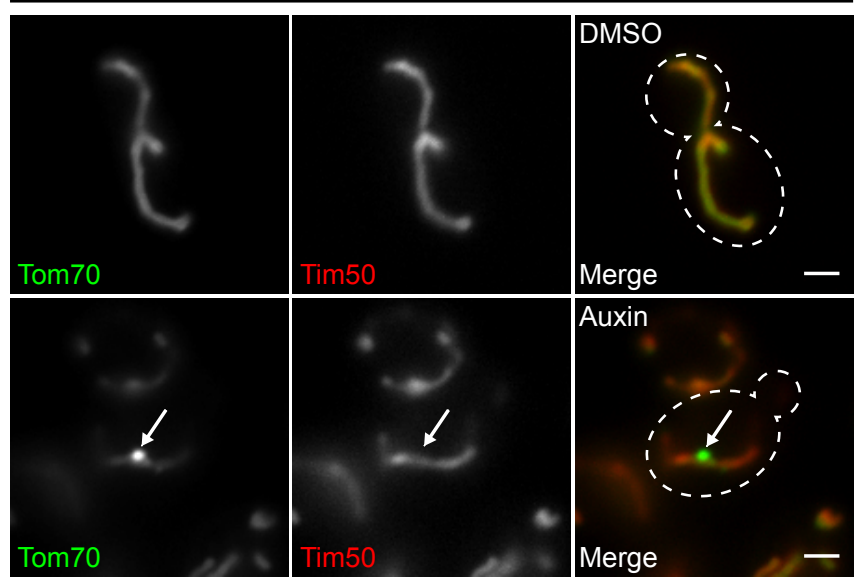

E

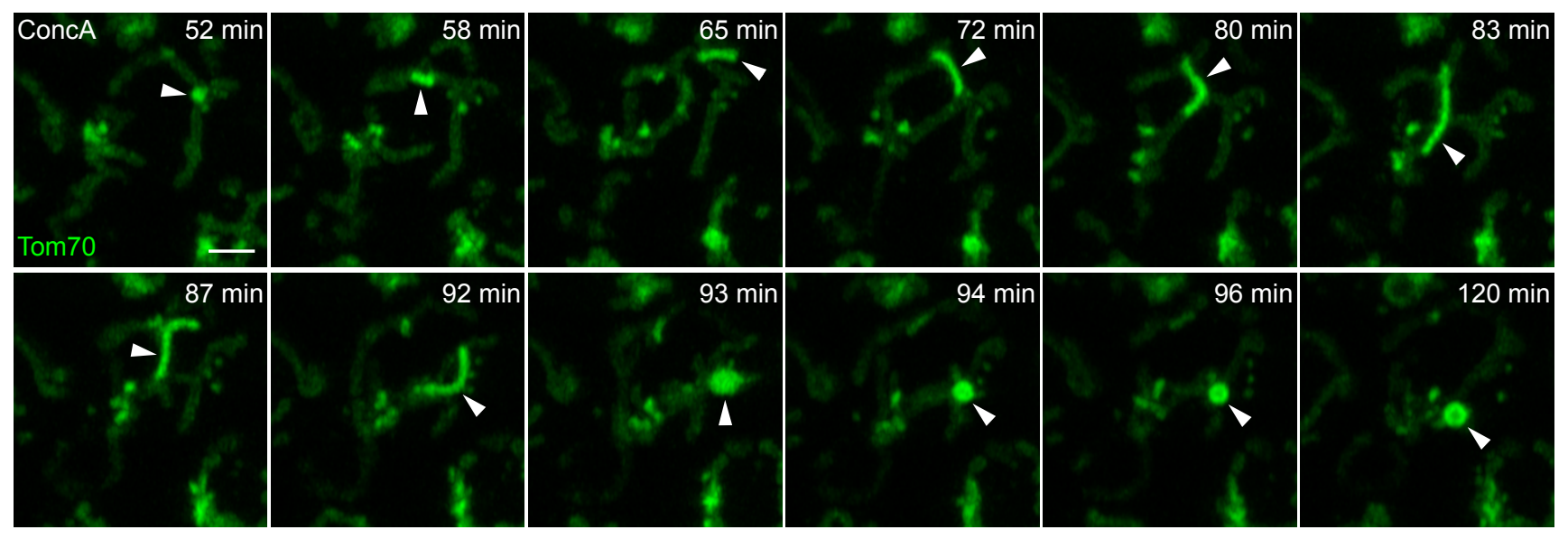


MoRxiv preprint doi: https://doi.org/10.1101/202(B3.13.991091; this version posted March 14, 2020. The copyright holder for this preprint (which was not certified by peer review) is the author/funder, who has granted bioRxiv a license to display the preprint in perpetuity. It is made

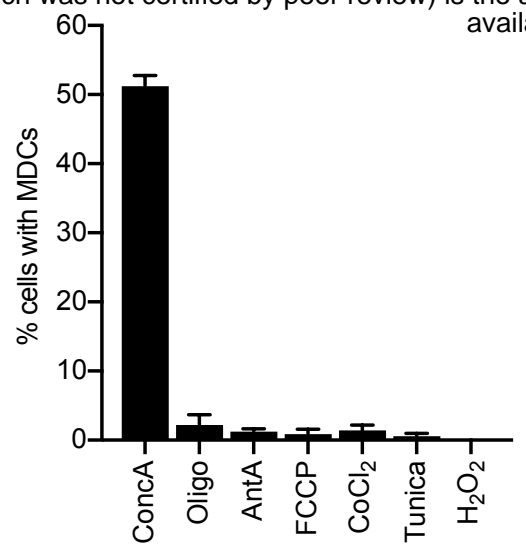
available under

\section{C}
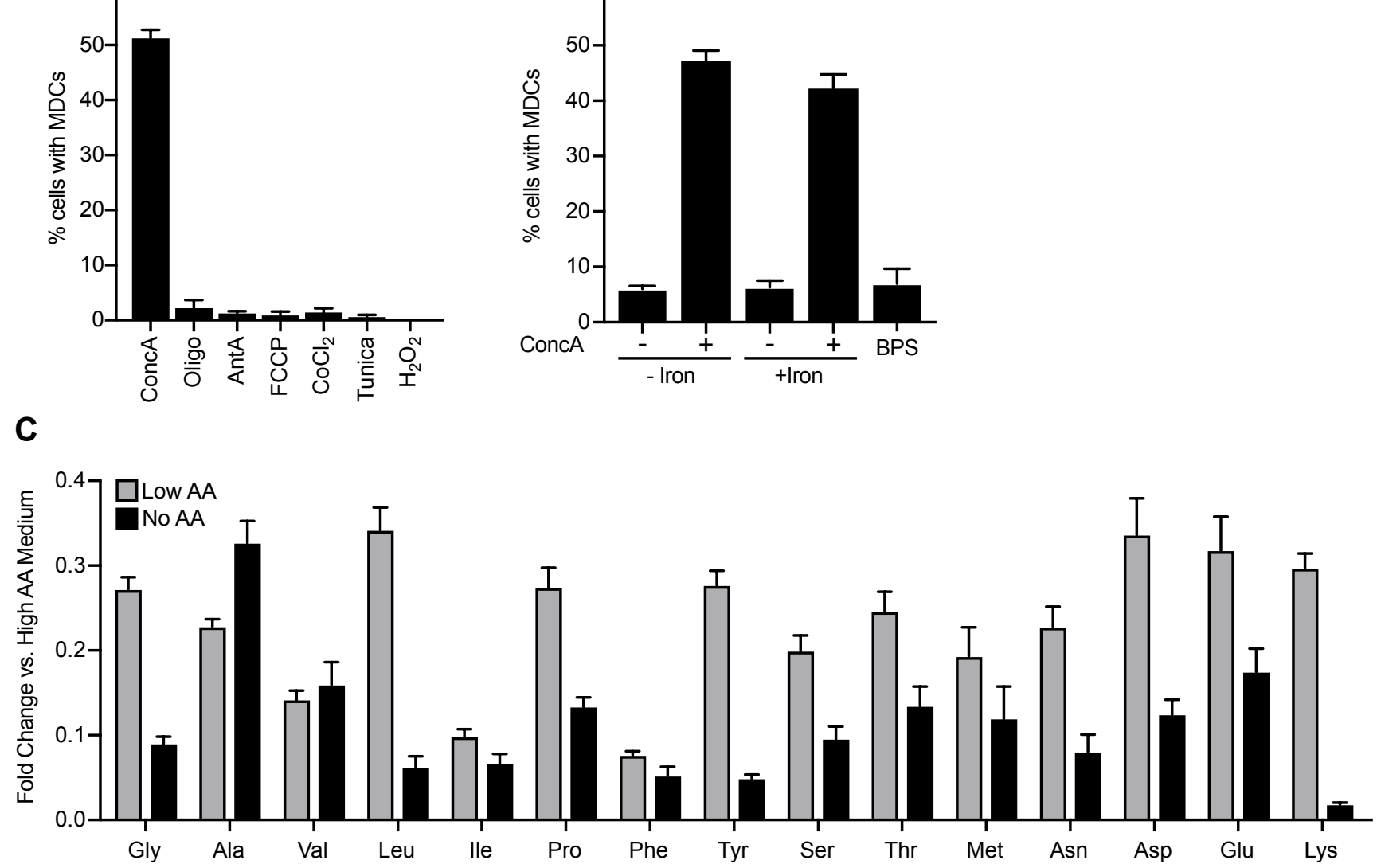

D
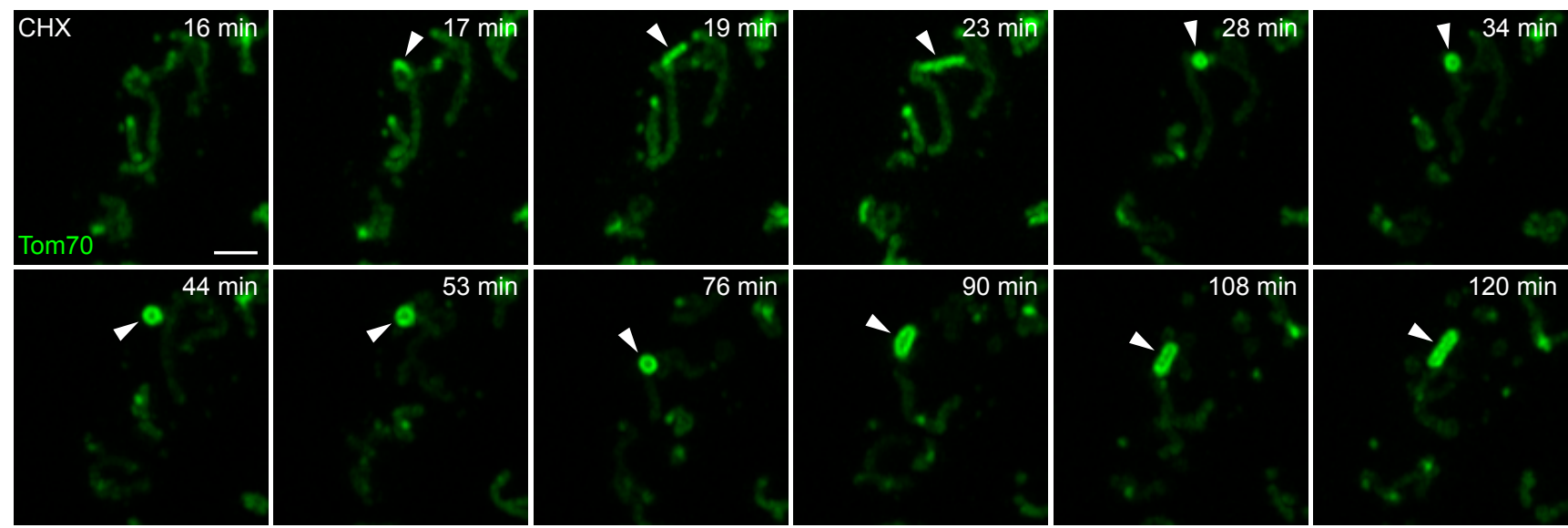

$\mathbf{E}$

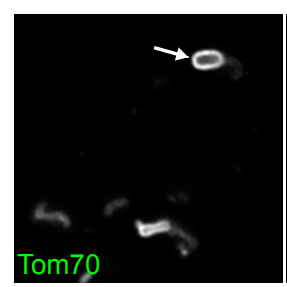

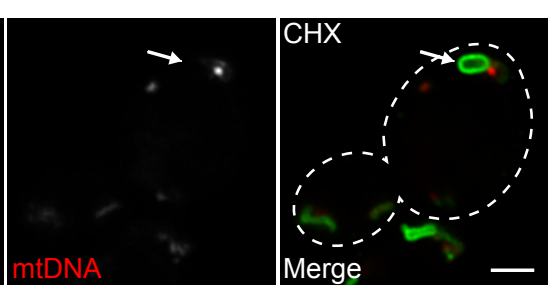

$\mathbf{F}$
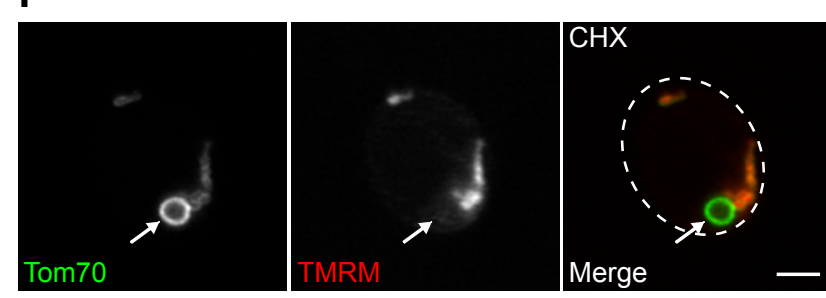
AjoRxiv preprint doi: https://doi.org/10.1101/2020.03.13.991091; this ver\$m posted March 14, 2020. The copyright holder for this preprint (which was not certified by peer review) is the author/funder, who has granted bioRxiv a license to display the preprint in perpetuity. It is made

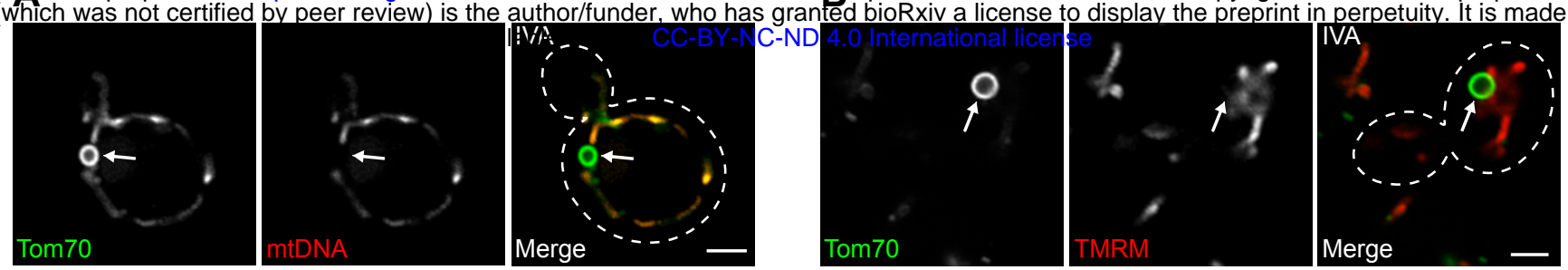

C

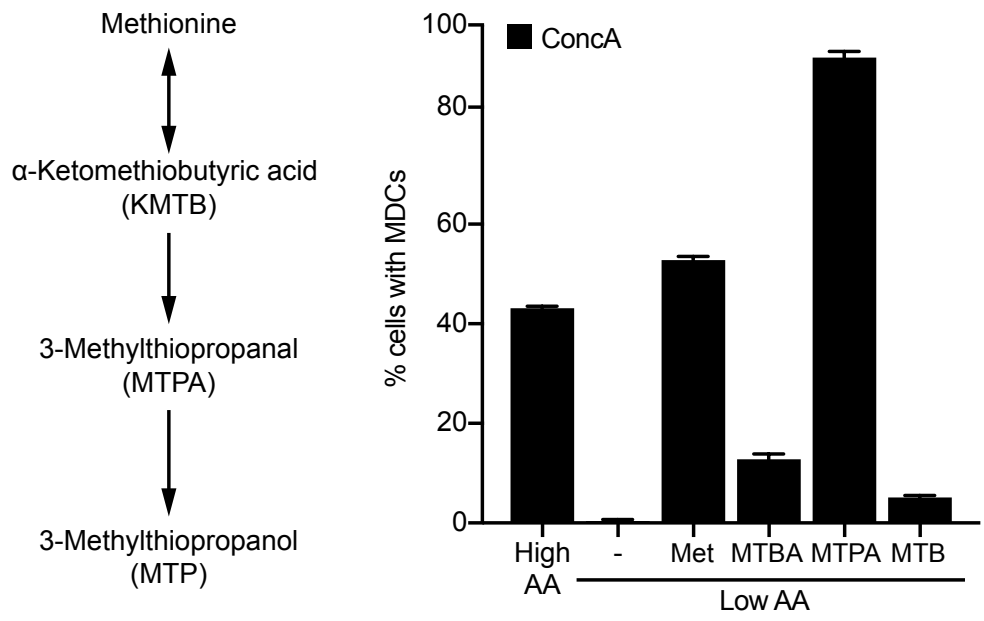

D

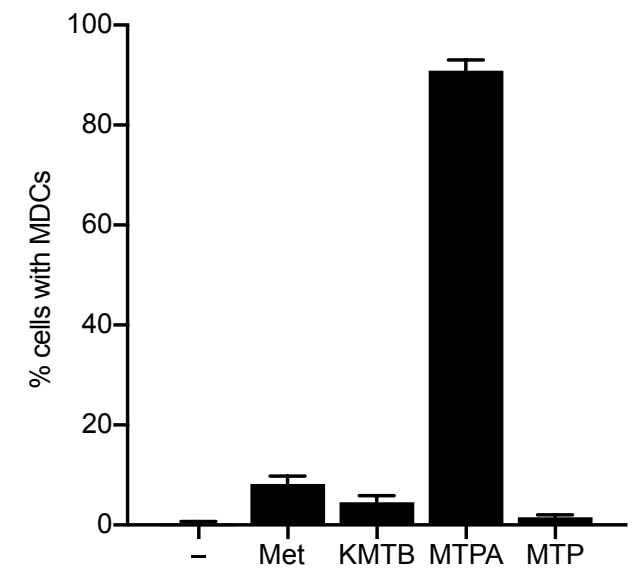

E
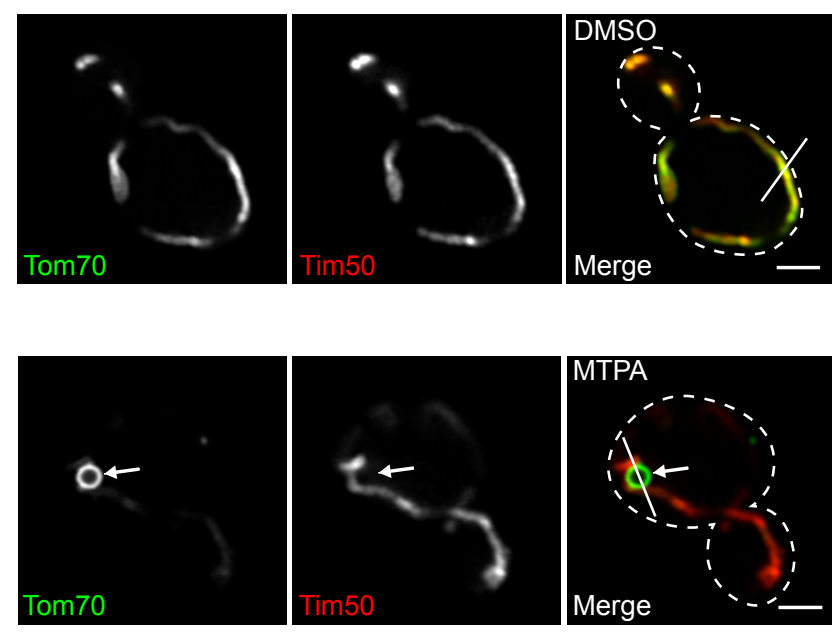

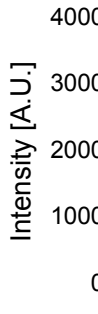
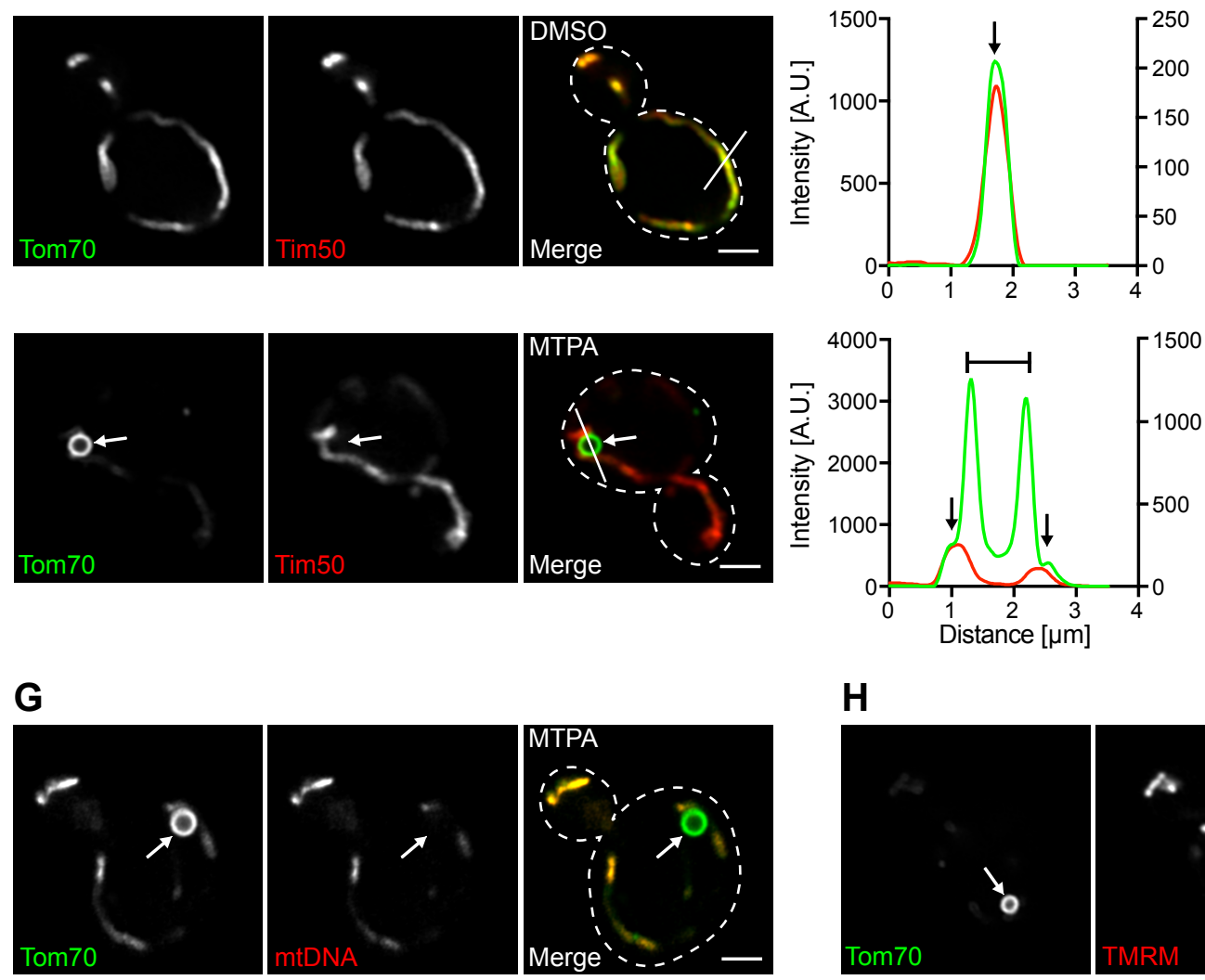
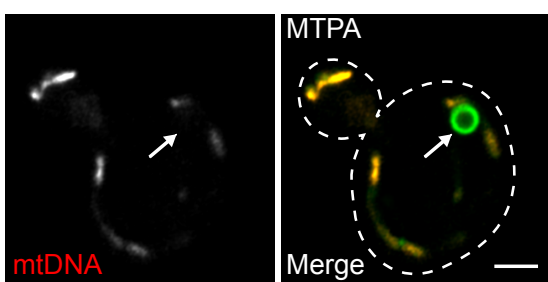

H

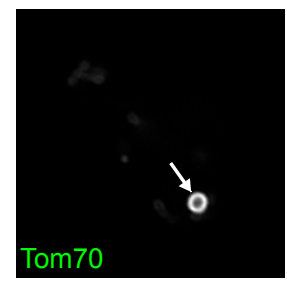

$\mathbf{F}$

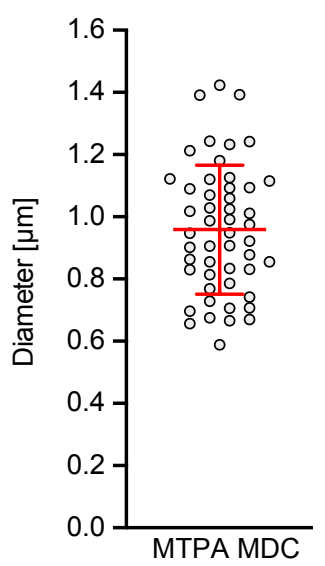


AioRxiv preprint doi: https://doi.org/10.1101/2020.03.13.991091; this vesion posted March 14, 2020. The copyright holder for this preprint

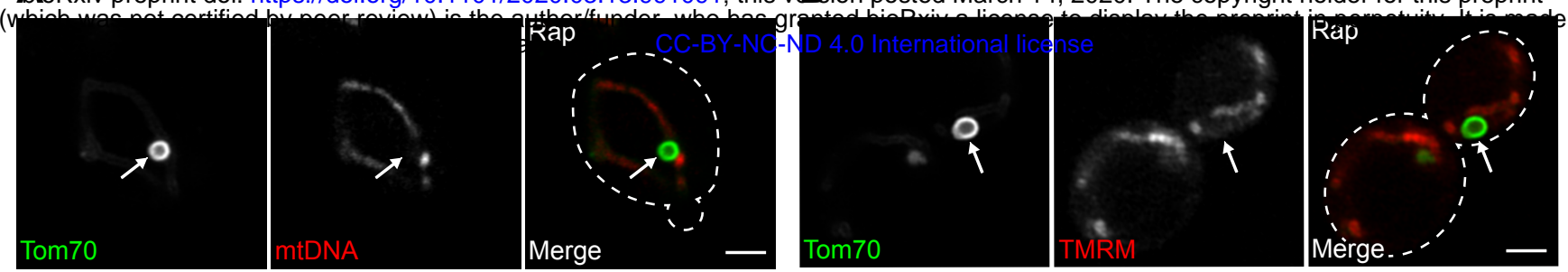

C

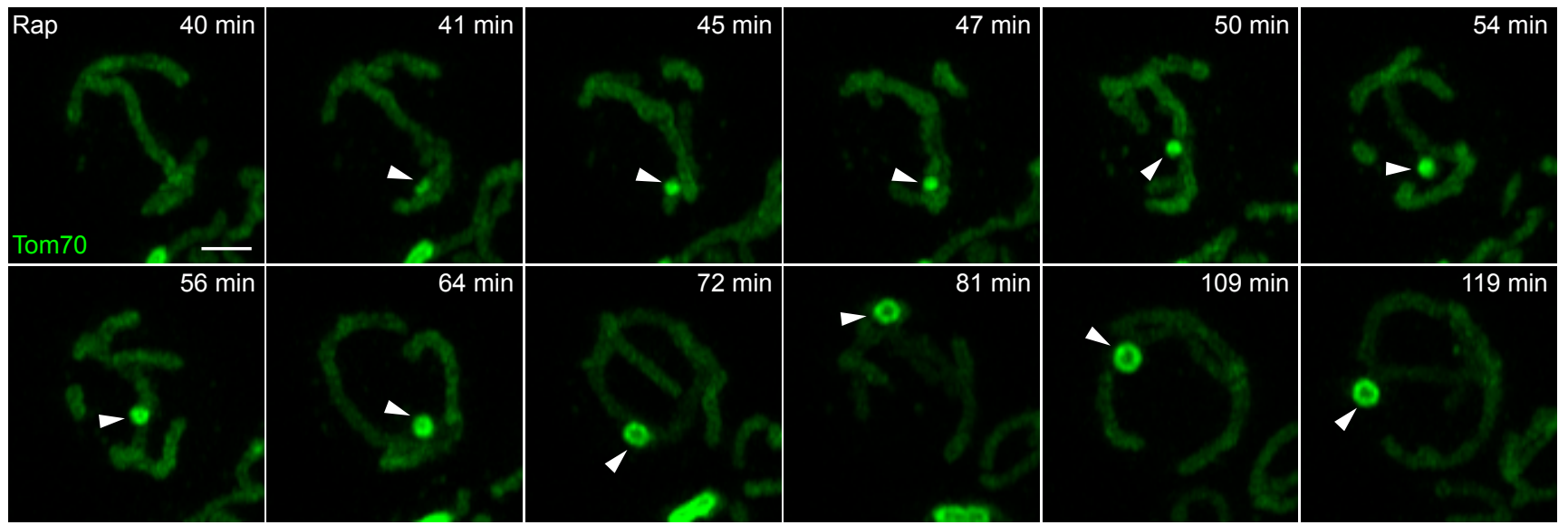

D
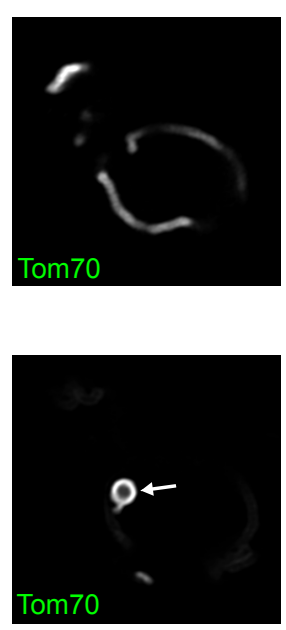

F

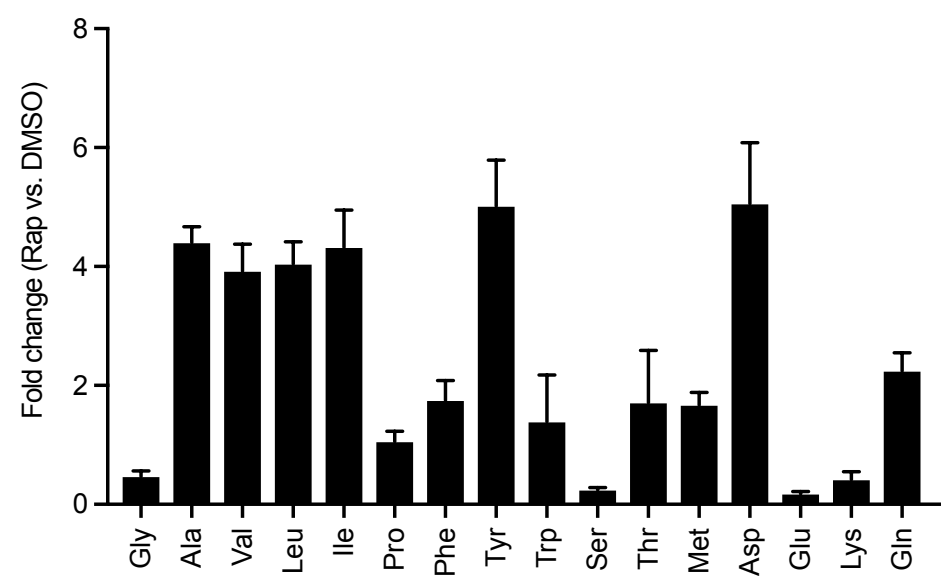

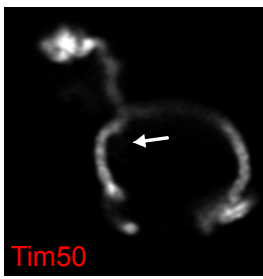
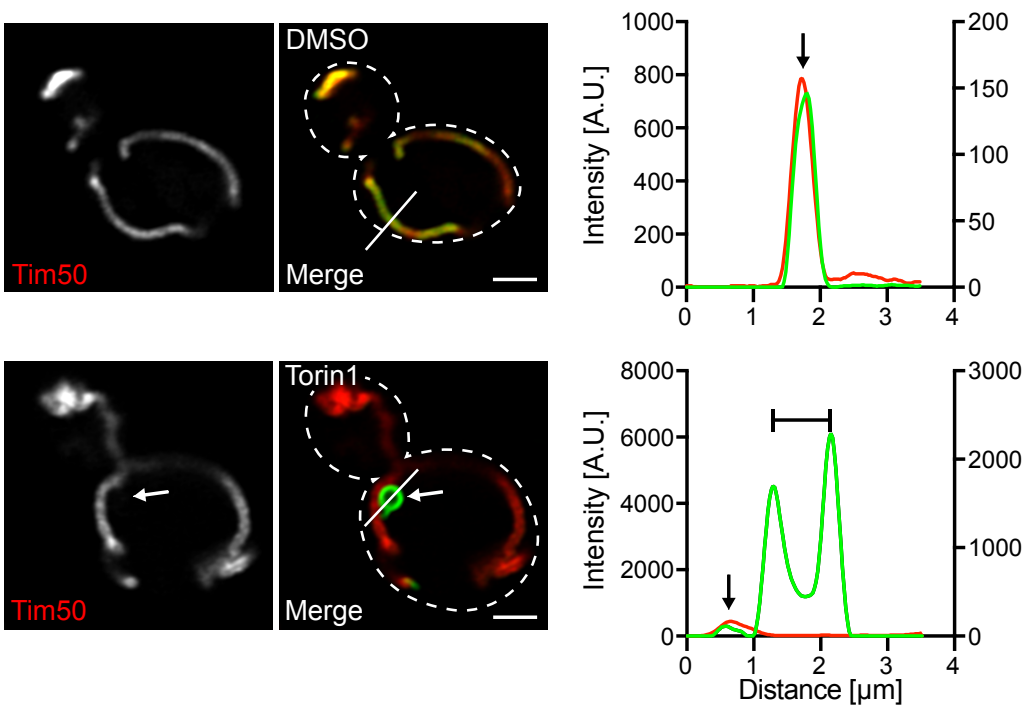

E

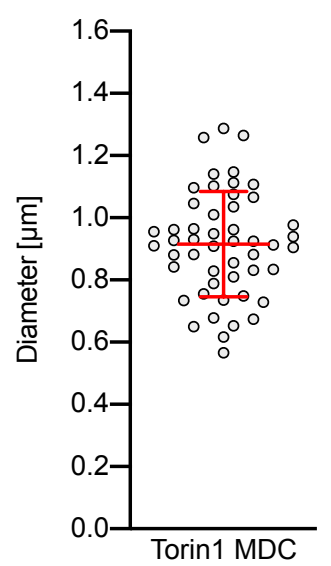


ajoRxiv preprint doi: https://doi.org/10.1101/2020.03.13.991091; this version po\$d March 14,2020 . The copyright holder for this preprint (which was not certified by peer review) is the author/funder, who has granted biokxiv a license to display the preprint in perpetuity. It is made
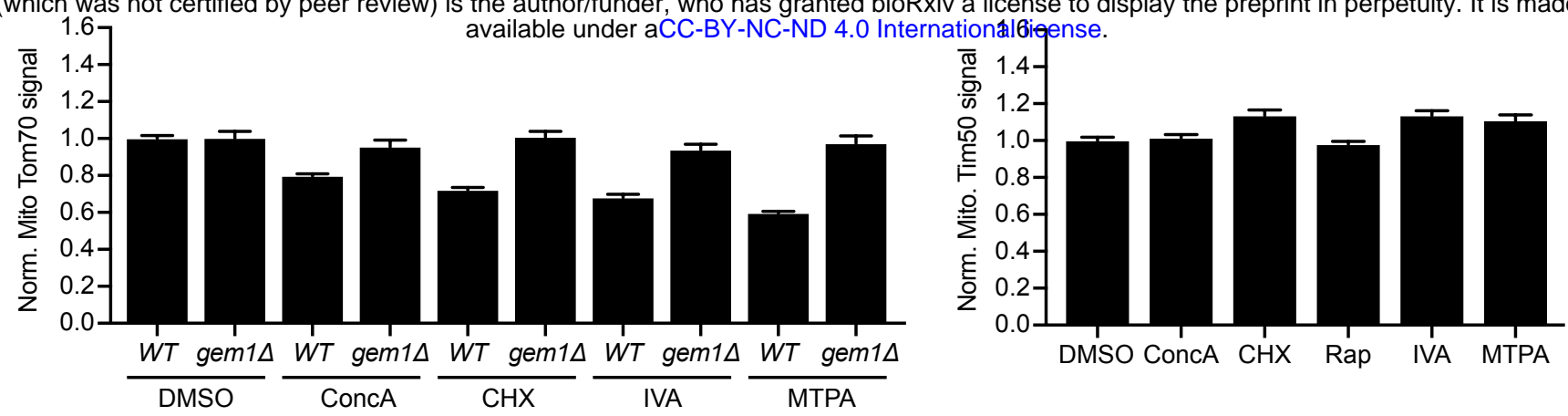

C
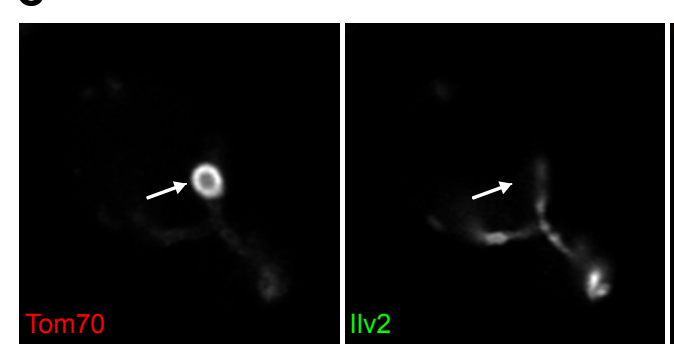

E

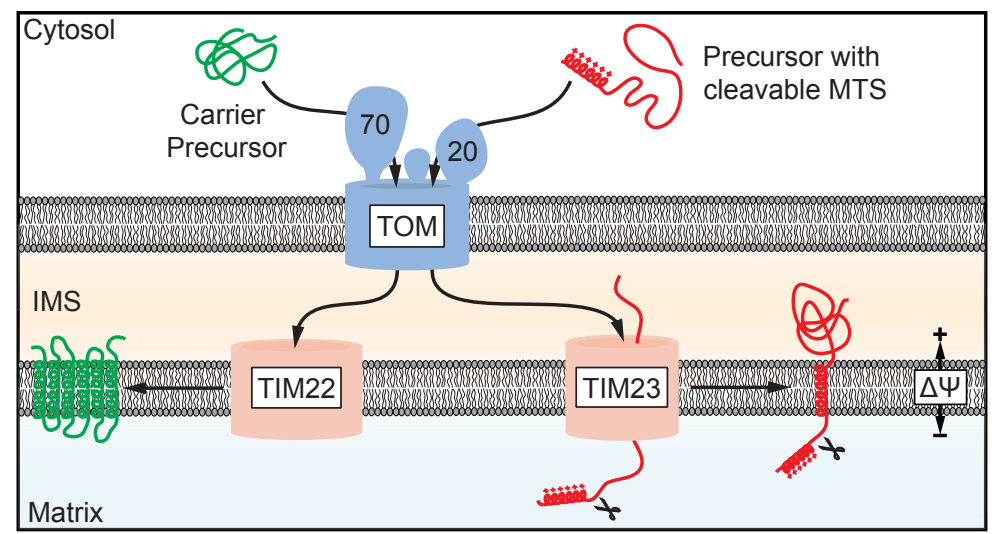

D

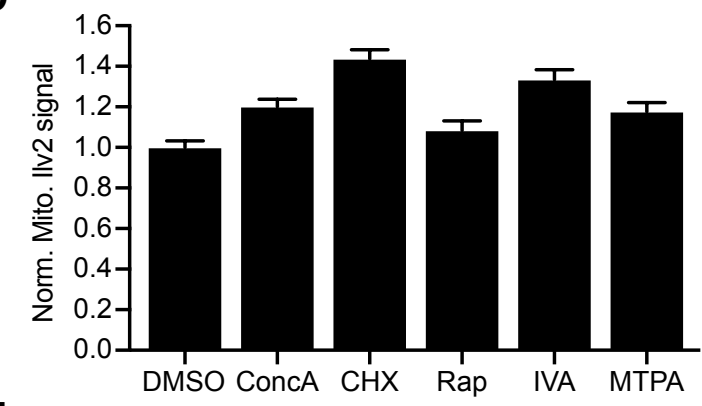

F

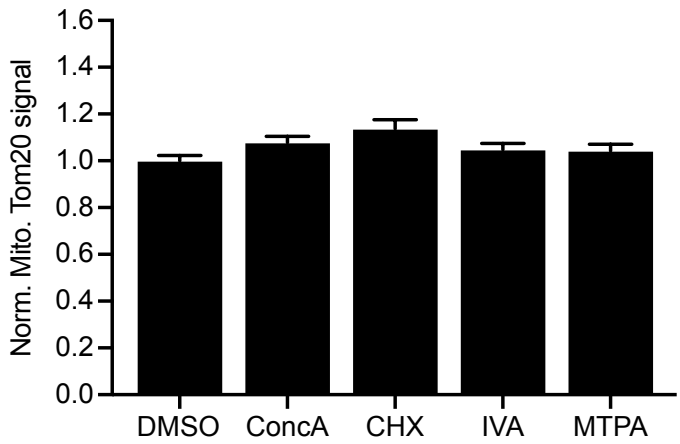


AbRxiv preprint doi: https://doi.org/10.1101/2020.03.13.991091; this version posted March 14, 2020. The copyright holder for this preprint (which 60 a

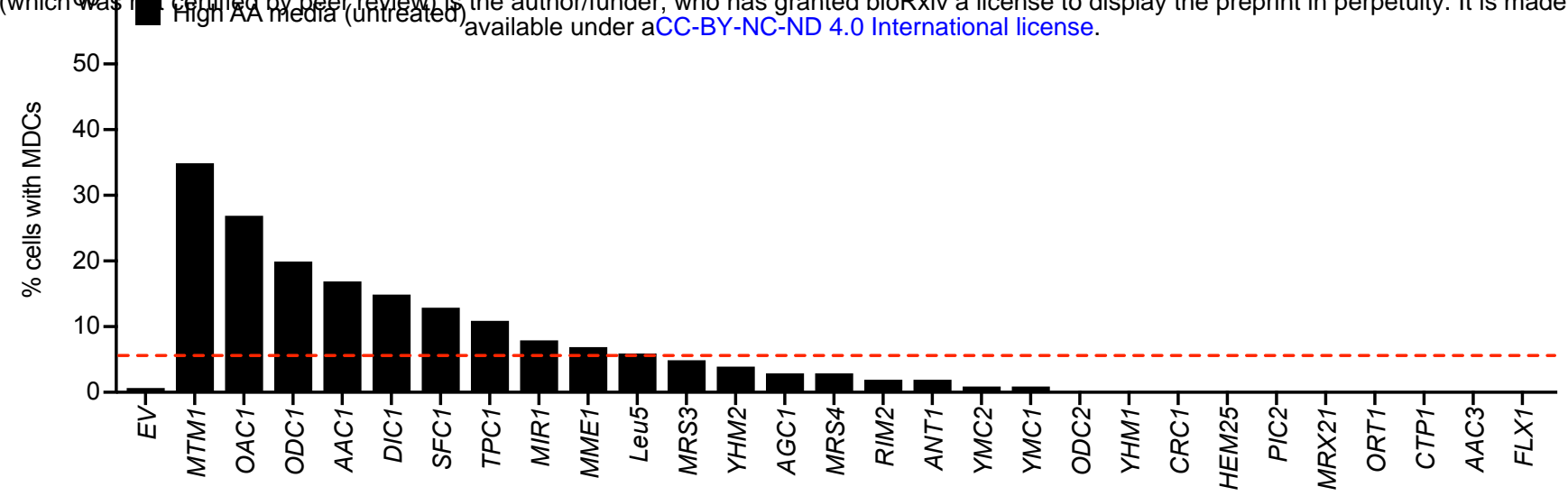

B

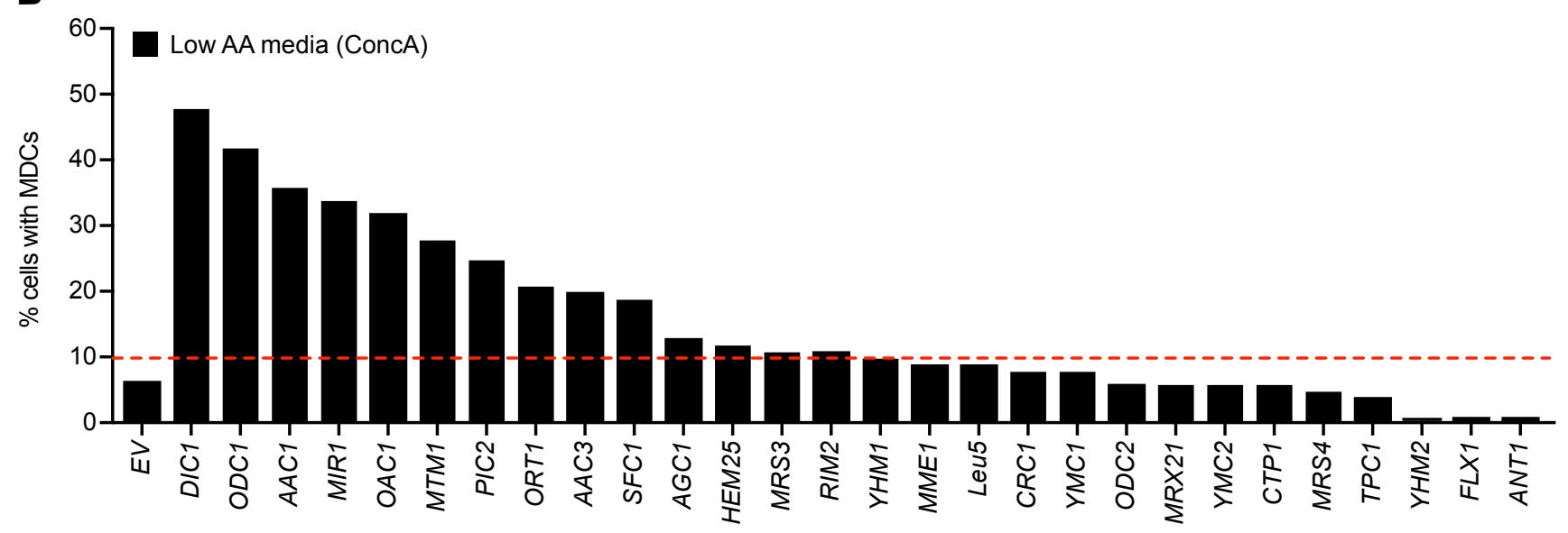

C

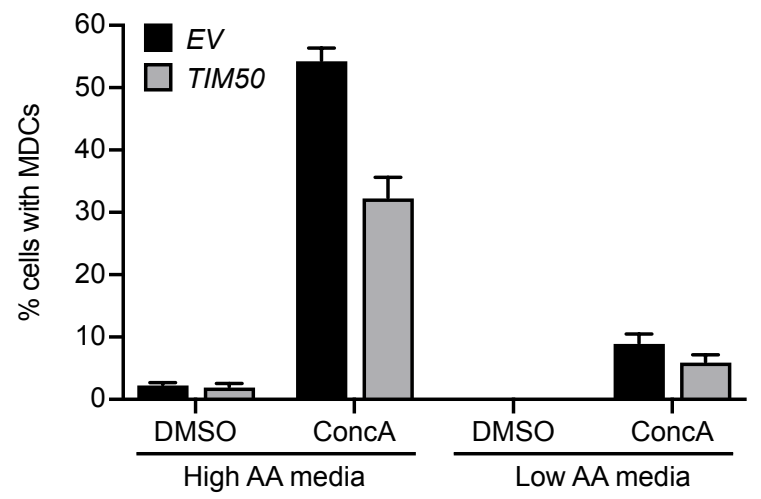


AbRxiv preprint doi: $h t$ tips:/Ldgiorg/10,1101/2020.03.13.991091; this ver\$Bn posted March 14, 2020. The copyright holder for this preprint

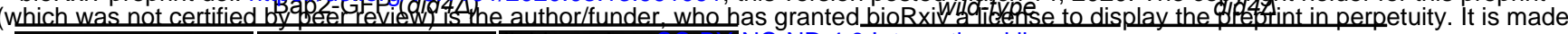

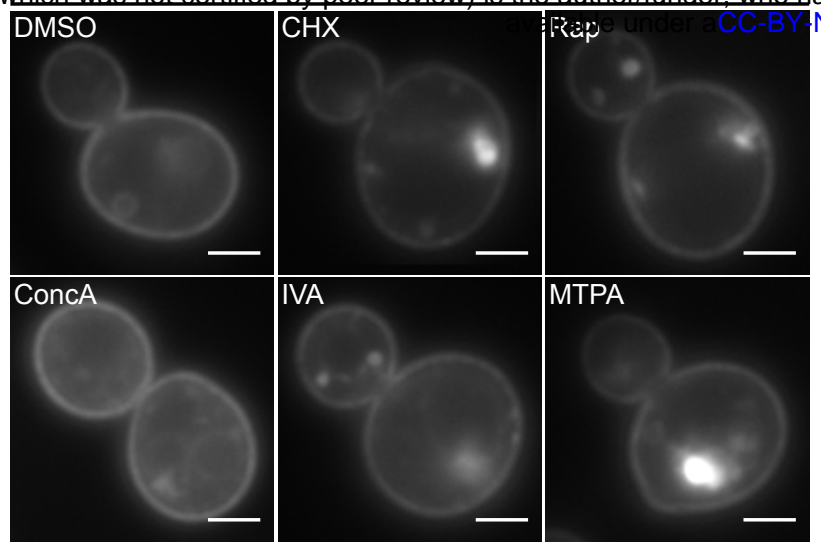

C

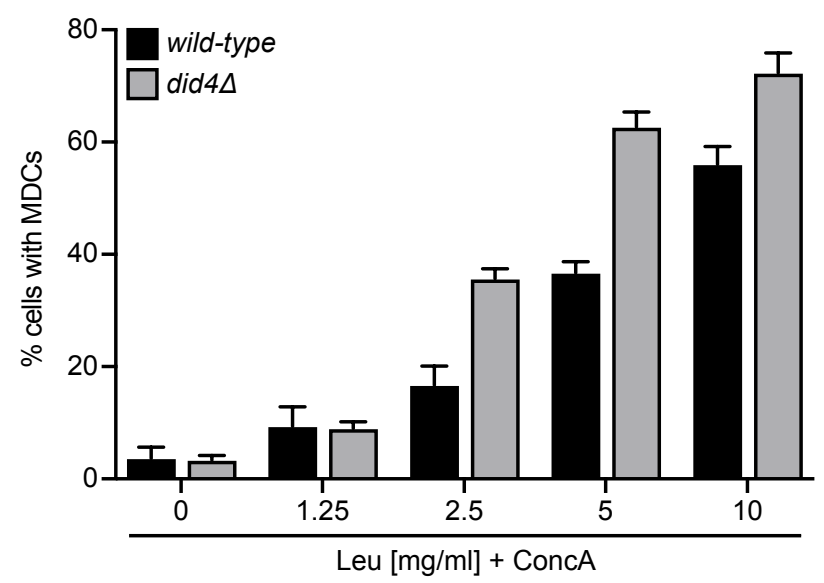

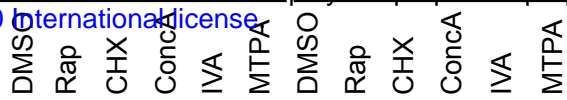

- Bap2-GFP

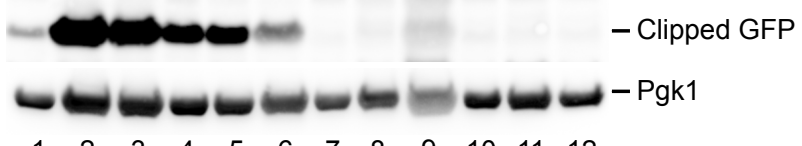

D

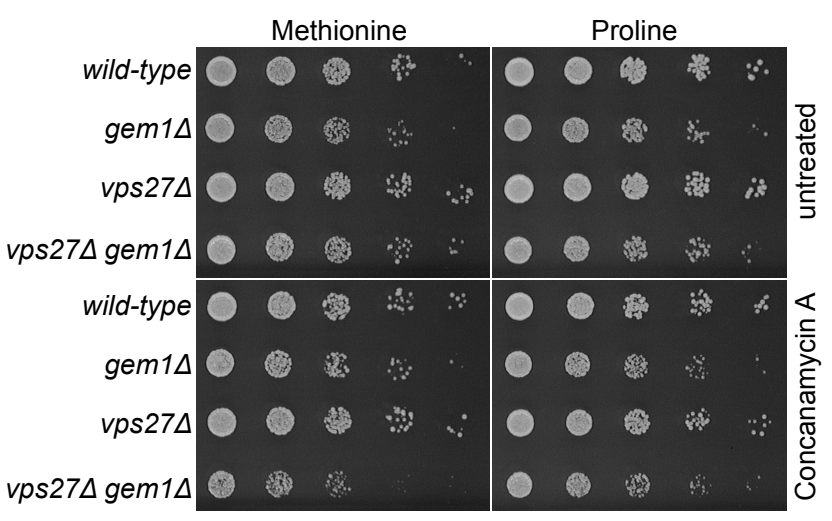

E

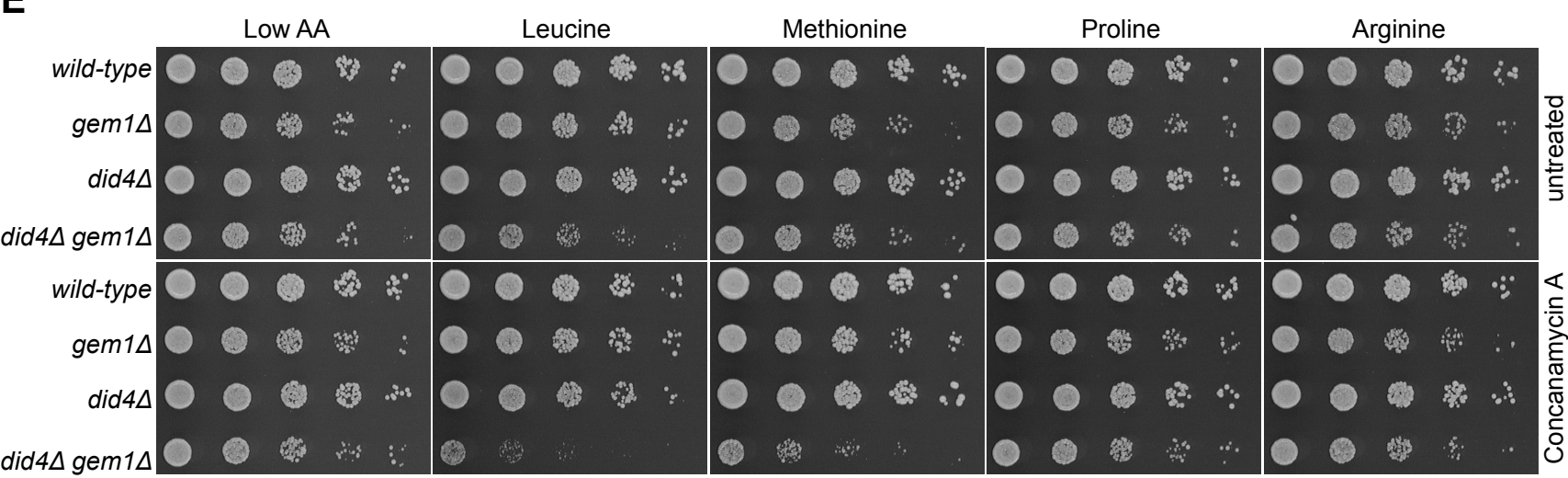




\section{KEY RESOURCES TABLE}

\begin{tabular}{|c|c|c|}
\hline REAGENT or RESOURCE & SOURCE & IDENTIFIER \\
\hline \multicolumn{3}{|l|}{ Antibodies } \\
\hline Rabbit polyclonal anti-FLAG affinity purified & Sigma-Aldrich & $\begin{array}{l}\text { Cat \# F7425; } \\
\text { RRID:AB_439687 }\end{array}$ \\
\hline Mouse monoclonal anti-PGK1 clone 22C5D8 & Abcam & $\begin{array}{l}\text { Cat \# ab113687; } \\
\text { RRID:AB } 10861977\end{array}$ \\
\hline Mouse monoclonal anti-GFP clones 7.1 and 13.1 & Roche & $\begin{array}{l}\text { Cat \# 11814460001; } \\
\text { RRID:AB_390913 }\end{array}$ \\
\hline \multicolumn{3}{|l|}{ Bacterial and Virus Strains } \\
\hline S. cerevisiae ORF collection (pDONR201/221) & $\begin{array}{l}\text { Harvard Institute of } \\
\text { Proteomics }\end{array}$ & $\mathrm{N} / \mathrm{A}$ \\
\hline Escherichia coli DH5a & N/A & $\mathrm{N} / \mathrm{A}$ \\
\hline \multicolumn{3}{|l|}{ Chemicals, Peptides, and Recombinant Proteins } \\
\hline$\alpha$-Ketomethiobutyric acid (KMTB) & Sigma-Aldrich & $\begin{array}{l}\text { Cat \# K6000; CAS \# } \\
595-37-9\end{array}$ \\
\hline 3-Indoleacetic acid (Auxin) & Sigma-Aldrich & $\begin{array}{l}\text { Cat \# I3750 ; CAS \# } \\
87-51-4\end{array}$ \\
\hline 3-Methylthiopropanal (MTPA) & Sigma-Aldrich & $\begin{array}{l}\text { Cat \# 277460; CAS } \\
\text { \# 123-38-6 }\end{array}$ \\
\hline 3-Methylthiopropanol (MTP) & Sigma-Aldrich & $\begin{array}{l}\text { Cat \# 318396; CAS } \\
\text { \# 505-10-2 }\end{array}$ \\
\hline 4,6-Diamidino-2-phenylindole dihydrochloride (DAPI) & ThermoFisher & $\begin{array}{l}\text { Cat \# D1306; CAS \# } \\
47165-04-8\end{array}$ \\
\hline Ammonium Iron(II) Sulfate Hexahydrate & Sigma-Aldrich & $\begin{array}{l}\text { Cat \# 215406; CAS } \\
\text { \# 7783-85-9 }\end{array}$ \\
\hline Antimycin A & Sigma-Aldrich & $\begin{array}{l}\text { Cat \# A8674; CAS \# } \\
1397-94-0\end{array}$ \\
\hline $\begin{array}{l}\text { Bathophenanthrolinedisulfonic Acid Disodium Salt } \\
\text { Hydrate (BPS) }\end{array}$ & Sigma-Aldrich & $\begin{array}{l}\text { Cat \# 146617; CAS } \\
\text { \# 52746-49-3 }\end{array}$ \\
\hline $\begin{array}{l}\text { Carbonyl cyanide 4-(trifluoromethoxy)phenylhydrazone } \\
\text { (FCCP) }\end{array}$ & Sigma-Aldrich & $\begin{array}{l}\text { Cat \# C2920; CAS \# } \\
370-86-5\end{array}$ \\
\hline Casamino acids & US Biological & $\begin{array}{l}\text { Cat \# 0012501A; } \\
\text { CAS \# 65072-00-6 }\end{array}$ \\
\hline Cobalt(II) chloride hexahydrate $\left(\mathrm{CoCl}_{2}\right)$ & Sigma-Aldrich & $\begin{array}{l}\text { Cat \# C8661; CAS \# } \\
7791-13-1\end{array}$ \\
\hline Concanamycin A & $\begin{array}{l}\text { Santa Cruz } \\
\text { Biotechnology }\end{array}$ & $\begin{array}{l}\text { Cat \# sc-202111; } \\
\text { CAS \# 80890-47-7 }\end{array}$ \\
\hline Concanavalin A & Sigma-Aldrich & $\begin{array}{l}\text { Cat \# L7647; CAS \# } \\
11028-71-0\end{array}$ \\
\hline Cycloheximide & Sigma-Aldrich & $\begin{array}{l}\text { Cat \# C1988; CAS \# } \\
66-81-9\end{array}$ \\
\hline Dimethyl sulfoxide (DMSO) & Sigma-Aldrich & $\begin{array}{l}\text { Cat \# D2650; CAS \# } \\
67-68-5\end{array}$ \\
\hline Hydrogen Peroxide $\left(\mathrm{H}_{2} \mathrm{O}_{2}\right)$ & Sigma-Aldrich & $\begin{array}{l}\text { Cat \# H1009; CAS \# } \\
7722-84-1\end{array}$ \\
\hline Isoamylalcohol (IAA) & Sigma-Aldrich & $\begin{array}{l}\text { Cat \# W205702; } \\
\text { CAS \# 123-51-3 }\end{array}$ \\
\hline Isovaleraldehyde (IVA) & Sigma-Aldrich & $\begin{array}{l}\text { Cat \# 146455; CAS } \\
\text { \# 590-86-3 }\end{array}$ \\
\hline
\end{tabular}




\begin{tabular}{|c|c|c|}
\hline Ketoisocaproic acid (KIC) & Sigma-Aldrich & $\begin{array}{l}\text { Cat \# 68255; CAS \# } \\
816-66-0\end{array}$ \\
\hline Oligomycin A & Sigma-Aldrich & $\begin{array}{l}\text { Cat \# 75351; CAS \# } \\
1404-19-9\end{array}$ \\
\hline Rapamycin & LC Laboritories & $\begin{array}{l}\text { Cat \# R-5000; CAS } \\
\# 53123-88-9\end{array}$ \\
\hline Tetramethylrhodamine methyl ester perchlorate (TMRM) & ThermoFisher & $\begin{array}{l}\text { Cat \# T668; CAS \# } \\
115532-50-8\end{array}$ \\
\hline Torin1 & R\&D Systems & $\begin{array}{l}\text { Cat \# 4247; CAS \# } \\
1222998-36-8\end{array}$ \\
\hline Tunicamycin & Sigma-Aldrich & $\begin{array}{l}\text { Cat \# T7765; CAS \# } \\
66054-36-2\end{array}$ \\
\hline \multicolumn{3}{|l|}{ Critical Commercial Assays } \\
\hline Gateway LR Clonase II Enzyme Mix & Thermo Fisher & Cat \# 11791020 \\
\hline Bicinchoninic Acid Protein Assay & G Biosciences & Cat \# 786-844 \\
\hline \multicolumn{3}{|l|}{ Experimental Models: Organisms/Strains } \\
\hline BY4741 MATa his $3 \Delta 1$ leu $2 \Delta 0$ ura3 $\Delta 0$ met $15 \Delta 0$ & $\begin{array}{l}\text { Brachman et al., 1998; } \\
\text { ATCC }\end{array}$ & Cat \# 201388 \\
\hline $\begin{array}{l}\text { BY4743 MATa/MATa his } 3 \Delta 1 / \text { his } 3 \Delta 1 \text { leu2 } \Delta 0 / \text { leu2 } \Delta 0 \\
\text { ura3 } \Delta 0 / \text { ura3 } \Delta 0 \text { met } 15 \Delta 0 /++ \text { lys } 2 \Delta 0 /+\end{array}$ & $\begin{array}{l}\text { Brachman et al., 1998; } \\
\text { ATCC }\end{array}$ & Cat \# 201390 \\
\hline $\begin{array}{l}\text { BY4743 TOM70-yEGFP:KanMX/+ TIM50- } \\
\text { mCherry:KanMX/+ }\end{array}$ & This study & AHY1480 \\
\hline $\begin{array}{l}\text { BY4743 TOM70-yEGFP:KanMX/+ TIM50- } \\
\text { mCherry:KanMX/+ PHLUM }\end{array}$ & This study & AHY4706 \\
\hline $\begin{array}{l}\text { BY4743 TOM70-yEGFP:SpHIS5MX/TOM70- } \\
\text { yEGFP:SpHIS5MX TIM50-mCherry:KanMX/TIM50- } \\
\text { mCherry:KanMX }\end{array}$ & This study & AHY7053 \\
\hline $\begin{array}{l}\text { BY4743 TOM70-yEGFP:KanMX/TOM70-yEGFP:KanMX } \\
\text { TIM50-mCherry:KanMX/TIM50-mCherry:KanMX }\end{array}$ & This study & AHY7620 \\
\hline BY4743 TOM70-yEGFP:KanMX/TOM70-yEGFP:KanMX & This study & AHY5082 \\
\hline $\begin{array}{l}\text { BY4743 VMA2-6xFLAG:HygMX/VMA2-6xFLAG:HygMX } \\
\text { LEU2:PGPD1-OsTir1-TermcYc1/ leu2 } \Delta 0\end{array}$ & This study & AHY8355 \\
\hline $\begin{array}{l}\text { BY4743 VMA2-AID*-6xFLAG:HygMX/VMA2-AID*- } \\
\text { 6xFLAG:HygMX LEU2:PGPD1-OsTir1-Termcrc1/leu2 } \Delta 0\end{array}$ & This study & AHY8353 \\
\hline $\begin{array}{l}\text { BY4743 TOM70-yEGFP:KanMX/TOM70-yEGFP:KanMX } \\
\text { TIM50-mCherry:KanMX/TIM50-mCherry:KanMX VMA2- } \\
\text { 6xFLAG:HygMX/VMA2-6xFLAG:HygMX LEU2:PGPD1- } \\
\text { OsTir1-Termcrc1/leu2 } \Delta 0\end{array}$ & This study & AHY8322 \\
\hline $\begin{array}{l}\text { BY4743 TOM70-yEGFP:KanMX/TOM70-yEGFP:KanMX } \\
\text { TIM50-mCherry:KanMX/TIM50-mCherry:KanMX VMA2- } \\
\text { AID*-6xFLAG:HygMX/VMA2-AID*-6xFLAG:HygMX } \\
\text { LEU2:PGPD1-OsTir1-Termcrc1/leu2 } \Delta 0\end{array}$ & This study & AHY8320 \\
\hline $\begin{array}{l}\text { BY4743 TOM70-yEGFP:KanMX/TOM70-yEGFP:KanMX } \\
\text { TIM50-mCherry:KanMX/TIM50-mCherry:KanMX } \\
\text { gcn2 } \triangle:: \text { LEU2/gcn2 } \Delta:: L E U 2\end{array}$ & This study & AHY8090 \\
\hline $\begin{array}{l}\text { BY4743 TOM70-yEGFP:KanMX/TOM70-yEGFP:KanMX } \\
\text { TIM50-mCherry:KanMX/TIM50-mCherry:KanMX } \\
\text { gIn3 } \Delta:: \text { LEU2/gIn3 } \Delta:: \text { LEU2 }\end{array}$ & This study & AHY8092 \\
\hline $\begin{array}{l}\text { BY4743 TOM70-yEGFP:KanMX/TOM70-yEGFP:KanMX } \\
\text { TIM50-mCherry:KanMX/TIM50-mCherry:KanMX } \\
\text { ssy1 } \Delta:: \text { LEU2/ssy1 } \Delta:: \text { LEU2 }\end{array}$ & This study & AHY8094 \\
\hline $\begin{array}{l}\text { BY4743 TOM70-yEGFP:KanMX/TOM70-yEGFP:KanMX } \\
\text { TIM50-mCherry:KanMX/TIM50-mCherry:KanMX } \\
\text { gpa2 } \Delta:: \text { LEU2/gpa2A::LEU2 }\end{array}$ & This study & AHY8096 \\
\hline
\end{tabular}




\begin{tabular}{|c|c|c|}
\hline $\begin{array}{l}\text { BY4743 TOM70-yEGFP:KanMX/TOM70-yEGFP:KanMX } \\
\text { TIM50-mCherry:KanMX/TIM50-mCherry:KanMX } \\
\text { gpr1 } 1 \Delta: \text { :LEU2/gpr1 } 1:: \text { LEU2 }\end{array}$ & This study & AHY8098 \\
\hline $\begin{array}{l}\text { BY4743 TOM70-yEGFP:KanMX/+ TIM50- } \\
\text { mCherry:KanMX/+ gem1 } 1 \Delta: \text { HygMX/gem1 } 1: \text { HygMX }\end{array}$ & This study & $\mathrm{AHY} 4057$ \\
\hline $\begin{array}{l}\text { BY4743 TOM70-mCherry:KanMX/TOM70- } \\
\text { mCherry:KanMX TOM20-yEGFP:SpHIS5MX/TOM20- } \\
\text { yEGFP:SpHIS5MX }\end{array}$ & This study & AHY8531 \\
\hline $\begin{array}{l}\text { BY4743 TOM20-yEGFP:KanMX/+ TIM50- } \\
\text { mCherry:KanMX/+ }\end{array}$ & This study & AHY7804 \\
\hline $\begin{array}{l}\text { BY4743 TOM20-yEGFP:KanMX/+ TIM50- } \\
\text { mCherry:KanMX/+ gem1 } 1:: \text { HygMX/gem1 } 1: \text { HygMX }\end{array}$ & This study & AHY7806 \\
\hline $\begin{array}{l}\text { BY4743 TIM50-yEGFP:KanMX/+ TOM70- } \\
\text { mCherry:KanMX/+ }\end{array}$ & This study & AHY7816 \\
\hline $\begin{array}{l}\text { BY4743 ILV2-yEGFP:SpHIS5MX/+ TIM50- } \\
\text { mCherry:KanMX/+ }\end{array}$ & This study & AHY7808 \\
\hline $\begin{array}{l}\text { BY4743 ILV2-yEGFP:SpHIS5MX/ILV2- } \\
\text { yEGFP:SpHIS5MX TOM70-mCherry:KanMX/TOM70- } \\
\text { mCherry:KanMX }\end{array}$ & This study & AHY8529 \\
\hline $\begin{array}{l}\text { BY4743 Tcd2-yEGFP:KanMX/+ Tim50- } \\
\text { mCherry:KanMX/+ }\end{array}$ & This study & AHY9354 \\
\hline $\begin{array}{l}\text { BY4743 Tcd2-yEGFP:KanMX/+ Tim50- } \\
\text { mCherry:KanMX/+ tom70 } \Delta:: \text { URA3/tom70 }:: \text { URA3 }\end{array}$ & This study & AHY9356 \\
\hline $\begin{array}{l}\text { BY4743 Tcd2-yEGFP:KanMX/+ Tim50- } \\
\text { mCherry:KanMX/+ tom71 }:: \text { LEU2/tom71 }:: \text { LEU2 }\end{array}$ & This study & AHY9358 \\
\hline 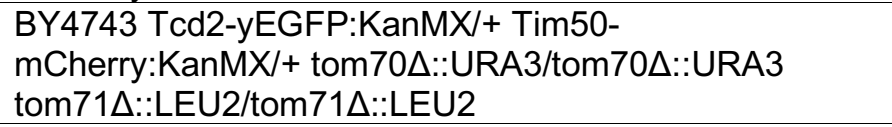 & This study & AHY9360 \\
\hline $\begin{array}{l}\text { BY4743 TOM70-yEGFP:KanMX/+ TIM50- } \\
\text { mCherry:KanMX/+ chr 1(199456-199457)::PGPD1-empty- } \\
\text { Termcrc1-URA3/+ }\end{array}$ & This study & AHY7624 \\
\hline $\begin{array}{l}\text { BY4743 TOM70-yEGFP:KanMX/+ TIM50- } \\
\text { mCherry:KanMX/+ chr 1(199456-199457)::PGPD1- } \\
\text { TOM70-Termcrc1-URA3/+ }\end{array}$ & This study & AHY9119 \\
\hline $\begin{array}{l}\text { BY4743 Tom70-yEGFP:KanMX/+ Tim50- } \\
\text { mCherry:KanMX/+ tom20 } \Delta:: \text { URA3/tom20 }:: \text { URA3 }\end{array}$ & This study & AHY10032 \\
\hline $\begin{array}{l}\text { BY4743 TOM70-yEGFP:KanMX/+ TIM50- } \\
\text { mCherry:KanMX/+ chr 1(199456-199457)::PGPD1- } \\
\text { TOM20-TermcYc1-URA3/+ }\end{array}$ & This study & AHY9632 \\
\hline $\begin{array}{l}\text { BY4743 TOM70-yEGFP:KanMX/+ TIM50- } \\
\text { mCherry:KanMX/+ chr 1(199456-199457)::PGPD1-TIM50- } \\
\text { Termcrc1-URA3/+ }\end{array}$ & This study & AHY9724 \\
\hline $\begin{array}{l}\text { BY4743 TOM70-yEGFP:KanMX/+ TIM50- } \\
\text { mCherry:KanMX/+ chr 1(199456-199457)::PGPD1-OAC1- } \\
\text { Termcrc1-URA3/+ }\end{array}$ & This study & AHY7850 \\
\hline $\begin{array}{l}\text { BY4743 TOM70-yEGFP:KanMX/+ TIM50- } \\
\text { mCherry:KanMX/+ chr 1(199456-199457)::PGPD1-AAC3- } \\
\text { TermcYc1-URA3/+ }\end{array}$ & This study & AHY7852 \\
\hline $\begin{array}{l}\text { BY4743 TOM70-yEGFP:KanMX/+ TIM50- } \\
\text { mCherry:KanMX/+ chr 1(199456-199457)::PGP1-RIM2- } \\
\text { TermcYc1-URA3/+ }\end{array}$ & This study & AHY7853 \\
\hline $\begin{array}{l}\text { BY4743 TOM70-yEGFP:KanMX/+ TIM50- } \\
\text { mCherry:KanMX/+ chr 1(199456-199457)::PGPD1-AGC1- } \\
\text { Termcrc1-URA3/+ }\end{array}$ & This study & AHY7854 \\
\hline
\end{tabular}




\begin{tabular}{|c|c|c|}
\hline $\begin{array}{l}\text { BY4743 TOM70-yEGFP:KanMX/+ TIM50- } \\
\text { mCherry:KanMX/+ chr 1(199456-199457)::PGPD1-FLX1- } \\
\text { TermcYc1-URA3/+ }\end{array}$ & This study & AHY7855 \\
\hline $\begin{array}{l}\text { BY4743 TOM70-yEGFP:KanMX/+ TIM50- } \\
\text { mCherry:KanMX/+ chr 1(199456-199457)::PGPD1-ANT1- } \\
\text { Termcyc1-URA3/+ }\end{array}$ & This study & AHY7864 \\
\hline $\begin{array}{l}\text { BY4743 TOM70-yEGFP:KanMX/+ TIM50- } \\
\text { mCherry:KanMX/+ chr 1(199456-199457)::PGPD1-MME1- } \\
\text { Termcrc1-URA3/+ }\end{array}$ & This study & AHY7865 \\
\hline $\begin{array}{l}\text { BY4743 TOM70-yEGFP:KanMX/+ TIM50- } \\
\text { mCherry:KanMX/+ chr 1(199456-199457)::PGPD1-LEU5- } \\
\text { Termcrc1-URA3/+ }\end{array}$ & This study & AHY7866 \\
\hline $\begin{array}{l}\text { BY4743 TOM70-yEGFP:KanMX/+ TIM50- } \\
\text { mCherry:KanMX/+ chr 1(199456-199457)::PGPD1-TPC1- } \\
\text { Termcrc1-URA3/+ }\end{array}$ & This study & AHY7867 \\
\hline $\begin{array}{l}\text { BY4743 TOM70-yEGFP:KanMX/+ TIM50- } \\
\text { mCherry:KanMX/+ chr 1(199456-199457)::PGPD1-SFC1- } \\
\text { Termcrc1-URA3/+ }\end{array}$ & This study & AHY7868 \\
\hline $\begin{array}{l}\text { BY4743 TOM70-yEGFP:KanMX/+ TIM50- } \\
\text { mCherry:KanMX/+ chr 1(199456-199457)::PGPD1-ODC2- } \\
\text { Termcrc1-URA3/+ }\end{array}$ & This study & AHY7869 \\
\hline $\begin{array}{l}\text { BY4743 TOM70-yEGFP:KanMX/+ TIM50- } \\
\text { mCherry:KanMX/+ chr 1(199456-199457)::PGPD1-YHM1- } \\
\text { Termcrc1-URA3/+ }\end{array}$ & This study & AHY7870 \\
\hline $\begin{array}{l}\text { BY4743 TOM70-yEGFP:KanMX/+ TIM50- } \\
\text { mCherry:KanMX/+ chr 1(199456-199457)::PGPD1-YHM2- } \\
\text { Termcrc1-URA3/+ }\end{array}$ & This study & AHY7884 \\
\hline $\begin{array}{l}\text { BY4743 TOM70-yEGFP:KanMX/+ TIM50- } \\
\text { mCherry:KanMX/+ chr 1(199456-199457)::PGPD1-CRC1- } \\
\text { Termcrc1-URA3/+ }\end{array}$ & This study & AHY7885 \\
\hline $\begin{array}{l}\text { BY4743 TOM70-yEGFP:KanMX/+ TIM50- } \\
\text { mCherry:KanMX/+ chr 1(199456-199457)::PGPD1-YMC1- } \\
\text { Termcrc1-URA3/+ }\end{array}$ & This study & AHY7886 \\
\hline $\begin{array}{l}\text { BY4743 TOM70-yEGFP:KanMX/+ TIM50- } \\
\text { mCherry:KanMX/+ chr 1(199456-199457)::PGPD1-ODC1- } \\
\text { Termcrc1-URA3/+ }\end{array}$ & This study & AHY7887 \\
\hline $\begin{array}{l}\text { BY4743 TOM70-yEGFP:KanMX/+ TIM50- } \\
\text { mCherry:KanMX/+ chr 1(199456-199457)::PGPD1-DIC1- } \\
\text { Termcrc1-URA3/+ }\end{array}$ & This study & AHY7888 \\
\hline $\begin{array}{l}\text { BY4743 TOM70-yEGFP:KanMX/+ TIM50- } \\
\text { mCherry:KanMX/+ chr 1(199456-199457)::PGPD1-MTM1- } \\
\text { Termcrc1-URA3/+ }\end{array}$ & This study & AHY7889 \\
\hline $\begin{array}{l}\text { BY4743 TOM70-yEGFP:KanMX/+ TIM50- } \\
\text { mCherry:KanMX/+ chr 1(199456-199457)::PGPD1- } \\
\text { HEM25-TermcYc1-URA3/+ }\end{array}$ & This study & AHY7890 \\
\hline $\begin{array}{l}\text { BY4743 TOM70-yEGFP:KanMX/+ TIM50- } \\
\text { mCherry:KanMX/+ chr 1(199456-199457)::PGPD1-PIC2- } \\
\text { Termcrc1-URA3/+ }\end{array}$ & This study & AHY7891 \\
\hline $\begin{array}{l}\text { BY4743 TOM70-yEGFP:KanMX/+ TIM50- } \\
\text { mCherry:KanMX/+ chr 1(199456-199457)::PGPD1- } \\
\text { MRX21-TermcYC1-URA3/+ }\end{array}$ & This study & AHY7892 \\
\hline $\begin{array}{l}\text { BY4743 TOM70-yEGFP:KanMX/+ TIM50- } \\
\text { mCherry:KanMX/+ chr 1(199456-199457)::PGPD1-MIR1- } \\
\text { TermcYc1-URA3/+ }\end{array}$ & This study & AHY7893 \\
\hline
\end{tabular}




\begin{tabular}{|c|c|c|}
\hline $\begin{array}{l}\text { BY4743 TOM70-yEGFP:KanMX/+ TIM50- } \\
\text { mCherry:KanMX/+ chr 1(199456-199457)::PGPD1-MRS3- } \\
\text { TermcYc1-URA3/+ }\end{array}$ & This study & AHY7894 \\
\hline $\begin{array}{l}\text { BY4743 TOM70-yEGFP:KanMX/+ TIM50- } \\
\text { mCherry:KanMX/+ chr 1(199456-199457)::PGPD1-MRS4- } \\
\text { Termcrc1-URA3/+ }\end{array}$ & This study & AHY7895 \\
\hline $\begin{array}{l}\text { BY4743 TOM70-yEGFP:KanMX/+ TIM50- } \\
\text { mCherry:KanMX/+ chr 1(199456-199457)::PGPD1-ORT1- } \\
\text { Termcrc1-URA3/+ }\end{array}$ & This study & AHY7896 \\
\hline $\begin{array}{l}\text { BY4743 TOM70-yEGFP:KanMX/+ TIM50- } \\
\text { mCherry:KanMX/+ chr 1(199456-199457)::PGPD1-AAC1- } \\
\text { TermcYc1-URA3/+ }\end{array}$ & This study & AHY7897 \\
\hline $\begin{array}{l}\text { BY4743 TOM70-yEGFP:KanMX/+ TIM50- } \\
\text { mCherry:KanMX/+ chr 1(199456-199457)::PGPD1-YMC2- } \\
\text { Termcrc1-URA3/+ }\end{array}$ & This study & AHY7898 \\
\hline $\begin{array}{l}\text { BY4743 TOM70-yEGFP:KanMX/+ TIM50- } \\
\text { mCherry:KanMX/+ chr 1(199456-199457)::PGPD1-CTP1- } \\
\text { Termcrc1-URA3/+ }\end{array}$ & This study & AHY7899 \\
\hline BY4741 BAP2-yEGFP:KanMX & This study & AHY10005 \\
\hline BY4741 BAP2-yEGFP:KanMX vps270::URA3 & This study & AHY10154 \\
\hline BY4741 BAP2-yEGFP:KanMX did4A::URA3 & This study & AHY10156 \\
\hline BY4741 gem1 $\Delta:: H y g M X$ & This study & AHY4230 \\
\hline BY4741 vps27 $::$ KanMX & This study & AHY9873 \\
\hline BY4741 vps27 $\triangle::$ KanMX gem1 $1::$ HygMX & This study & AHY10041 \\
\hline BY4741 did4 $\Delta::$ KanMX & This study & AHY9913 \\
\hline BY4741 did4 $\triangle::$ KanMX gem1 $\Delta::$ HygMX & This study & AHY10145 \\
\hline \multicolumn{3}{|l|}{ Oligonucleotides } \\
\hline \multicolumn{3}{|l|}{ See Table S1 } \\
\hline \multicolumn{3}{|l|}{ Recombinant DNA } \\
\hline Plasmid: pRS410 & Addgene & Plasmid \# 11258 \\
\hline Plasmid: pRS40Hyg & Daniel Gottschling & N/A \\
\hline Plasmid: pRS306 & $\begin{array}{l}\text { Sikorski and Hieter, } \\
1989\end{array}$ & N/A \\
\hline Plasmid: pRS305 & $\begin{array}{l}\text { Sikorski and Hieter, } \\
1989\end{array}$ & N/A \\
\hline Plasmid: pKT128 & $\begin{array}{l}\text { Sheff and Thorn, } \\
\text { 2004; Addgene }\end{array}$ & Plasmid \# 8729 \\
\hline Plasmid: pKT127-mCherry & $\begin{array}{l}\text { Daniel Gottschling } \\
\text { (Calico) }\end{array}$ & N/A \\
\hline Plasmid: pKT127 & $\begin{array}{l}\text { Sheff and Thorn, } \\
\text { 2004; Addgene }\end{array}$ & Plasmid \# 8728 \\
\hline Plasmid: pHLUM & $\begin{array}{l}\text { Mülleder et al., 2012; } \\
\text { Addgene }\end{array}$ & Plasmid \# 40276 \\
\hline Plasmid: pAG306GPD-empty chr 1 & $\begin{array}{l}\text { Hughes and } \\
\text { Gottschling, } 2012\end{array}$ & N/A \\
\hline Plasmid: pAG306GPD-ccdB chr 1 & $\begin{array}{l}\text { Hughes and } \\
\text { Gottschling, } 2012\end{array}$ & N/A \\
\hline Plasmid: pHyg-6FLAG & This study & N/A \\
\hline Plasmid: pHyg-AID*-6FLAG & $\begin{array}{l}\text { Morawska and Ulrich, } \\
\text { 2013; Addgene }\end{array}$ & Plasmid \# 99519 \\
\hline Plasmid: pNH605-pGPD1-osTIR1 & Chan et al., 2018 & N/A \\
\hline Plasmid: pAG306GPD-AAC1 chr 1 & This study & $\mathrm{N} / \mathrm{A}$ \\
\hline
\end{tabular}




\begin{tabular}{|c|c|c|}
\hline Plasmid: pAG306GPD-AAC3 chr 1 & This study & N/A \\
\hline Plasmid: pAG306GPD-AGC1 chr 1 & This study & N/A \\
\hline Plasmid: pAG306GPD-ANT1 chr 1 & This study & N/A \\
\hline Plasmid: pAG306GPD-CRC1 chr 1 & This study & N/A \\
\hline Plasmid: pAG306GPD-CTP1 chr 1 & This study & N/A \\
\hline Plasmid: pAG306GPD-DIC1 chr 1 & This study & N/A \\
\hline Plasmid: pAG306GPD-FLX1 chr 1 & This study & N/A \\
\hline Plasmid: pAG306GPD-HEM25 chr 1 & This study & N/A \\
\hline Plasmid: pAG306GPD-LEU5 chr 1 & This study & N/A \\
\hline Plasmid: pAG306GPD-MIR1 chr 1 & This study & N/A \\
\hline Plasmid: pAG306GPD-MME1 chr 1 & This study & N/A \\
\hline Plasmid: pAG306GPD-MRS3 chr 1 & This study & $\mathrm{N} / \mathrm{A}$ \\
\hline Plasmid: pAG306GPD-MRS4 chr 1 & This study & N/A \\
\hline Plasmid: pAG306GPD-MRX21 chr 1 & This study & N/A \\
\hline Plasmid: pAG306GPD-MTM1 chr 1 & This study & N/A \\
\hline Plasmid: pAG306GPD-OAC1 chr 1 & This study & N/A \\
\hline Plasmid: pAG306GPD-ODC1 chr 1 & This study & N/A \\
\hline Plasmid: pAG306GPD-ODC2 chr 1 & This study & N/A \\
\hline Plasmid: pAG306GPD-ORT1 chr 1 & This study & N/A \\
\hline Plasmid: pAG306GPD-PIC2 chr 1 & This study & N/A \\
\hline Plasmid: pAG306GPD-RIM2 chr 1 & This study & N/A \\
\hline Plasmid: pAG306GPD-SFC1 chr 1 & This study & N/A \\
\hline Plasmid: pAG306GPD-TIM50 chr 1 & This study & N/A \\
\hline Plasmid: pAG306GPD-TOM20 chr 1 & This study & $\mathrm{N} / \mathrm{A}$ \\
\hline Plasmid: pAG306GPD-TOM70 chr 1 & This study & N/A \\
\hline Plasmid: pAG306GPD-TPC1 chr 1 & This study & N/A \\
\hline Plasmid: pAG306GPD-YHM1 chr 1 & This study & N/A \\
\hline Plasmid: pAG306GPD-YHM2 chr 1 & This study & N/A \\
\hline Plasmid: pAG306GPD-YMC1 chr 1 & This study & N/A \\
\hline Plasmid: pAG306GPD-YMC2 chr 1 & This study & N/A \\
\hline \multicolumn{3}{|l|}{ Software and Algorithms } \\
\hline FIJI & Schindelin et al., 2012 & Version 1 \\
\hline Prism & $\begin{array}{l}\text { GraphPad Software, } \\
\text { Inc. }\end{array}$ & Version 8 \\
\hline SnapGene & GSL Biotech & Version 4.2 \\
\hline ZEN Black Edition & Carl Zeiss Microscopy & Version 2.3 \\
\hline ZEN Blue Edition & Carl Zeiss Microscopy & Version 2.6 \\
\hline Photoshop CC & Adobe & Version 19 \\
\hline Image Lab & Bio-Rad & Version 6 \\
\hline MassHunter Qual & Agilent & Version B.07.00 \\
\hline MassHunter Quant & Agilent & Version B.07.00 \\
\hline
\end{tabular}

